Sludge Treatment Project Engineered Container Retrieval and Transfer System Preliminary Design Hazard Analysis Supplement 1

Prepared for the U.S. Department of Energy Assistant Secretary for Environmental Management

Contractor for the U.S. Department of Energy

under Contract DE-AC06-08RL14788

CH2MHILL

Plateau Romediation Company

P.O. Box 1600

Richland, Washington 99352 
PRC-STP-00473

Revision 0

EDC \#: ECR-11-001335

\section{Sludge Treatment Project Engineered Container Retrieval and Transfer System Preliminary Design Hazard Analysis Supplement 1}

Document Type: RPT

Program/Project: STP

G. R. Franz

R. H. Meichle

XRON

Date Published

July 2011

Prepared for the U.S. Department of Energy

Assistant Secretary for Environmental Management

Contractor for the U.S. Department of Energy

under Contract DE-AC06-08RL14788

CH2MHILL

Plateau Remadiation Company

P.O. Box 1600

Richland, Washington

$\frac{\operatorname{Nancy} A \text { Foned }}{7 / 18 / 20 l}$ Date

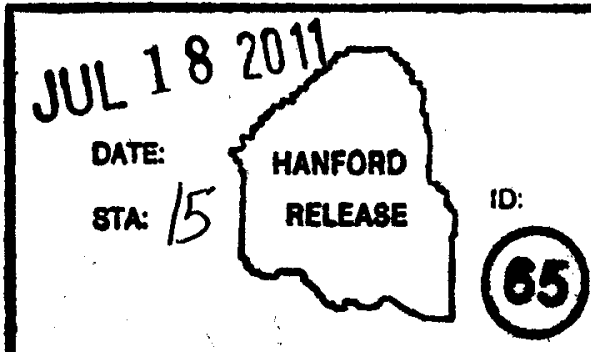

Release Stamp

Approved for Public Release;

Further Dissemination Unlimited 
PRC-STP-00473

Revision 0

TRADEMARK DISCLAMER

Reference herein to any specific commercial product, process,

or service by trade name, trademark, manufacturer, or

otherwise, does not necessarily constitute or imply its

endorsement, recommendation, or favoring by the United

States Government or any agency thereof or its contractors or subcontractors.

This report has been reproduced from the best available copy.

Printed in the United States of America

Total Pages: $\quad 83$ 


\section{CONTENTS}

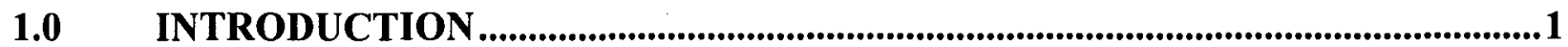

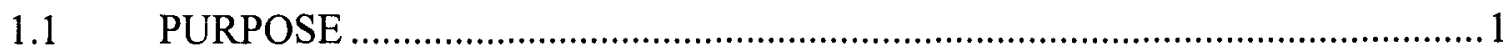

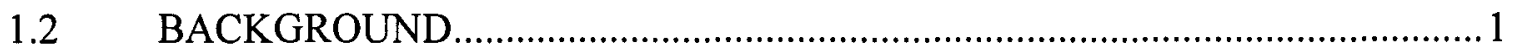

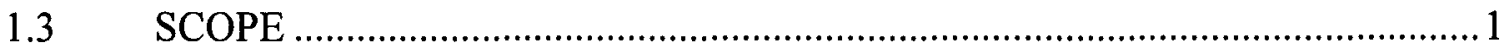

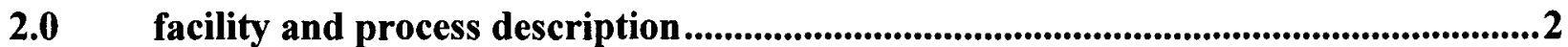

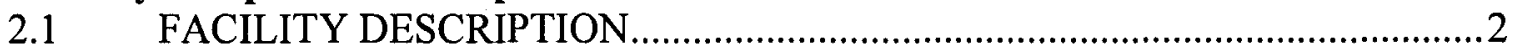

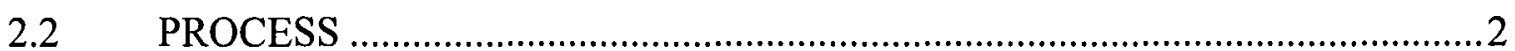

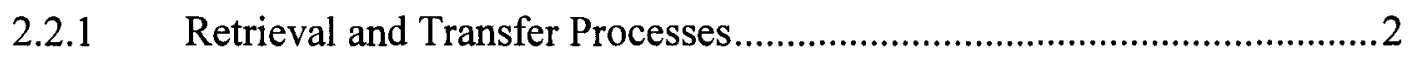

2.2.2 Operation Sequences (Gap Analysis) ....................................................2

3.0 METHODOLOGY

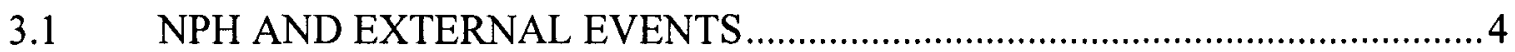

3.2 OPERATION SEQUENCES (GAP ANALYSIS),...........................................

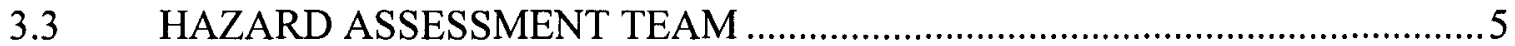

3.4 UNMITIGATED FREQUENCY AND CONSEQUENCE ESTIMATES............5

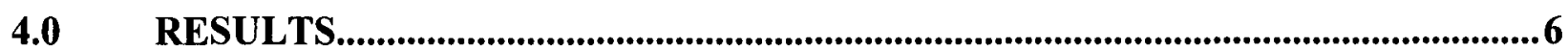

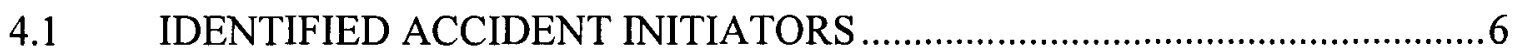

4.2 IDENTIFIED UNMITIGATED HAZARDOUS CONDITIONS ….....................7

$4.3 \quad$ FREQUENCY AND CONSEQUENCE LEVELS ...............................................

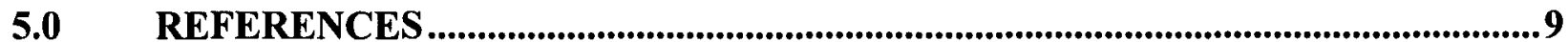

\section{APPENDICES}

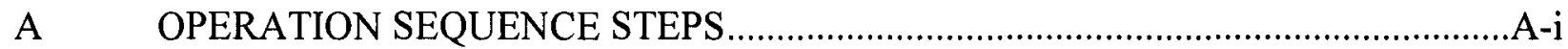

B "WHAT/IF" HAZARD ANALYSIS MEETING PARTICIPANTS ................................ B-i

C "WHAT/IF" HAZARD ANALYSIS RESULTS - NPH AND EXTERNAL EVENTS.

D "WHAT/IF" HAZARD ANALYSIS RESULTS - OPERATION SEQUENCES

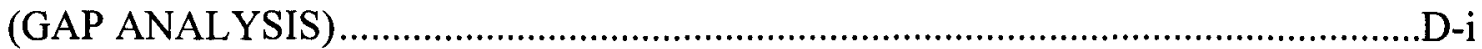

E FREQUENCY AND CONSEQUENCE LEVEL RESULTS ...................................... E-i

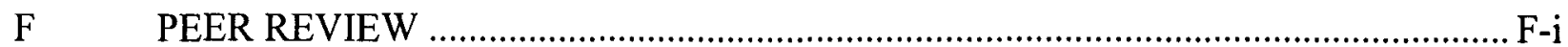


PRC-STP-00473 REV 0

\section{TABLES}

Table 1 - List of ECRTS NPH and External Events Considered in Hazards Analysis ....................4

Table 2 - Collocated Worker and Offsite Public Consequence Levels* .......................................5

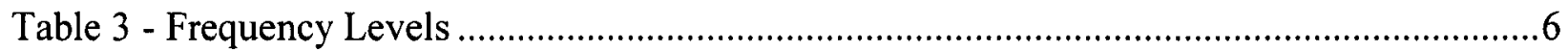

Table 4 - Guidance for SDC \& Other NPH Criteria Based on Unmitigated NPH Induced

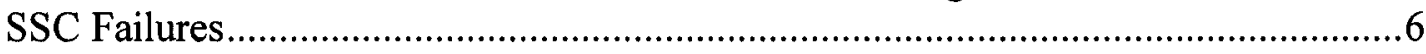

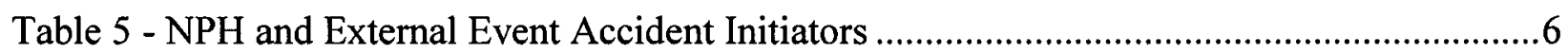

Table 6 - Basis for Appendix E Frequency and Consequence Levels................................................8 


\section{TERMS}

DOE-RL

ECRTS

HAZOP

LOEP

$\mathrm{NPH}$

NS

PDR

PC

SDC

SDS

STS

STSC
U.S. Department of Energy Richland Operations Office Engineered Container Retrieval and Transfer System Hazard and Operability Study

Loss of Electric Power

Natural Phenomena Hazards

Nuclear Safety

Preliminary Design Review

Performance Category

Seismic Design Criteria

Safety Design Strategy

Sludge Transport System

Sludge Transport and Storage Container 
PRC-STP-00473 REV 0

\subsection{INTRODUCTION}

\subsection{PURPOSE}

This "What/If" Hazards Analysis addresses hazards affecting the Sludge Treatment Project Engineered Container Retrieval and Transfer System (ECRTS) NPH and external events at the preliminary design stage. In addition, the hazards of the operation sequence steps for the mechanical handling operations in preparation of Sludge Transport and Storage Container (STSC), disconnect STSC and prepare STSC and Sludge Transport System (STS) for shipping are addressed.

This analysis provides a supplement to PRC-STP-00467, "Sludge Treatment Project Engineered Container Retrieval And Transfer System Preliminary Design Hazard And Operability Study".

\subsection{BACKGROUND}

The Engineered Container Retrieval and Transfer System (ECRTS) is being designed to retrieve sludge from six containers in the KW Basin for transfer to STSCs in the KW Basin Modified Annex. The ECRTS is summarized in PRC-STP-00467 and additional descriptions can be found in HNF-41051. PRC-STP-00467 provides the follow-up to the "What/If" Hazard Analysis for the Conceptual Design, PRC-STP-00124 "Sludge Treatment Project Engineered Container Retrieval And Transfer System Conceptual Design Hazards Analysis" and HAZOP for the draft preliminary design, PRC-STP-00346, "Sludge Treatment Project Engineered Container Retrieval And Transfer System Draft Preliminary Design Hazard And Operability Study".

The HAZOP methodology does not lend itself well to NPH and external events hazards analysis or consideration of the operation sequences steps involving personnel access to the modified annex and interaction with the installed equipment. The latter was referred to the Gap Analysis in the hazard analysis meetings. The NPH and External Event and Operation Sequence hazard analysis for the draft preliminary design status was captured in an initial Hazard Analyses on September 10, October 19, and October 22 and is documented in PRC-STP-00382, "Sludge Treatment Project Engineered Container Retrieval And Transfer System Draft Preliminary Design Hazard Analysis Supplement I".

PRC-STP-00409, 2011, "Sludge Treatment Project Engineered Container Retrieval and Transfer System Draft Preliminary Design Control Decision" documented the control decision for the draft preliminary design.

\subsection{SCOPE}

This document provides the "What/If" hazards analysis of NPH, external events and selected operation sequences for the ECRTS preliminary design to supplement the HAZOP provided PRC-STP-00467. The process conditions covered were:

- Retrieving sludge from an Engineered Container

- Transferring the slurry to an STSC 
- Decanting the STSC

- Backwashing the Sand Filter into the STSC

- Purging the STSC with nitrogen

- Purging the STS cask with nitrogen

- Pressurizing the STS cask with nitrogen

- Adding flocculant to the slurry during transfer

- Adding flocculant to the supernate

- Automatically flushing the transfer line

- Recovering excess sludge from an over-filled STSC

\subsection{FACILITY AND PROCESS DESCRIPTION}

\subsection{FACILITY DESCRIPTION}

The facility description is provided in PRC-STP-00467 which includes ECRTS systems in 105 KW Basin, Shielded Hose Chase, and Modified KW Basin Annex including Process Flow Diagrams (PFDs) and P\&IDs.

\subsection{PROCESS}

\subsubsection{Retrieval and Transfer Processes}

Sludge transfer to STSC, Flush sequence, Settle/Flocculate, Decant, Filter Backwash and Overfill Recovery processes are described in PRC-STP-00467 including Process Flow Diagrams (PFDs) and P\&IDs.

\subsubsection{Operation Sequences (Gap Analysis)}

In addition to the retrieval and transfer processes of section 2.2.1 above the Operation Modes from PRC-STP-00358, "Operating Sequence and Interlock Definition Document for the Engineered Container Retrieval and Transfer System (ECRTS)" which were analyzed are described as follows.

Mode 1: Prepare STSC. The process begins with receipt of an empty STSC in the STS Cask on the STS transport trailer. The trailer is positioned on the truck scale in the annex and disconnected from the tractor, which then exits the annex. The as-received weight is recorded, the cask is vented, the lid is removed and a radiological survey is performed. Exposed surfaces are covered with contamination control measures (plastic wrap, glove bags, etc.) as needed and the slurry transfer, decant, and ventilation hoses and instrument cables are attached to their respective STSC nozzles and instrument connections. Weight and level instrument readings are recorded continuously on a data logger. 


\section{PRC-STP-00473 REV 0}

Mode 8: Disconnect STSC. When the weight and level measurements in the decanted STSC indicate that the transferred sludge quantity is within the established process control limits, the final flush sequence is performed. The operators enter the annex building and align the valves to flush the sludge transfer and decant connector hoses with IXM water from the valve box to the STSC to reduce contamination levels and adjust the liquid level in the STSC to the specified final fill height. After flushing, the fill hose and decant hose are drained by opening the vent valves in the valve boxes. The hose connectors are unbolted and raised about one inch to open the vent port inside the connector. This allows air to flow from the vent valve through the inner hose to the connector and into the STSC, blowing out any residual liquid to prevent drips. If desired, the system can be left in this configuration for an extended time to dry the interior of the hoses. The hose connectors are then disconnected, the hose nozzle caps and STSC nozzle blind flanges are installed, and the hose connectors are placed in the storage position on the annex mezzanine. The ventilation hose is disconnected and capped. The inert gas system valve is opened, the inlet breather filter is valved off, and the STSC head space is purged. The purge hoses are disconnected and capped, and replaced with Nucfil ${ }^{\mathbb{1} 1}$ vent filters, and the final level reading is obtained before the instrument cables are disconnected from the STSC. Contamination control measures are removed and an exit radiological survey is conducted. Decontamination is performed if needed and the STS cask lid is installed.

Mode 9: Prepare for Shipping. After the STS cask lid is secured, the final shipping weight is measured and the shipping manifest is prepared. The vent port tools are installed on the cask and the cask is vented, purged with inert gas, and leak tested in preparation for transport. The annex door is opened, the tractor is connected, and the trailer removed from the annex. Shipping papers are verified and the STS is transported to T Plant.

\subsection{METHODOLOGY}

The purpose of this document is to provide input on the hazards and their consequences resulting from NPH, external events, and operation sequences to the control decisions for determination of necessary prevention and/or mitigation SSCs or administrative controls for ECRTS preliminary design. This document contains the NPH and External Events What-If/Checklist portion of the hazards analysis as well as the what-if operating sequences evaluations for modes 1,8 , and 9 . These are the modes when the K Basin Modified Annex will be occupied, and the focus has been on the potential to initiate radiological control events as well as to review for significant process fault initiators.

The results of the What-if meetings are documented in tabular form in Appendices $\mathrm{C}$ and $\mathrm{D}$. The table column headings are as follows:

Identifier: Unique identifier assigned to each What-if question. For NPH and external events, the identifier is the first character or series of characters of the event designation from the list in Table 1, followed by a hyphen and the number of the what-if within the event. For the gap

\footnotetext{
${ }^{1}$ NucFil@ is a registered trademark of Nuclear Filter Technology
} 
analysis based on the operating nodes, the first character is the mode, then a hyphen followed by the second character which is the activity within the mode, followed by the unique event number. The first and second numbers come from Appendix A and are described in the table.

"What-If": A description of the event that is postulated by the review team.

Causes: This column in the NPH and External Event lists the specific phenomenon. In the gap analysis for modes 1,8 , and 9 , this column identifies the mechanism that may have allowed the What-if to occur.

Hazardous Condition: This column identifies the hazardous condition that was identified as a result of the What-if (if any). If no hazardous condition results, then the word None is indicated. For this hazards analysis, conditions that may result in a future uncontrolled release event are identified with the initiating What-if as well as the subsequent operating activity that actually results in the release. As an example, a valve postulated to be left closed resulting in an overpressure and spray or splash and splatter when a transfer is initiated, would show as a hazardous condition both at the valve mis-positioning and at the pump starting.

Consequences: This column describes the consequence associated with the hazardous condition. If there is not a hazardous condition, then operational upset is indicated with corresponding cost and schedule impacts.

Candidate Controls: Candidate engineered and administrative controls are listed for those hazardous conditions that may result in consequences to onsite workers or off site public.

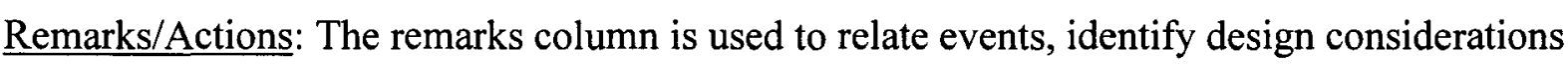
that have come up in the hazards review, and to carry actions for future work. These would be revised as the final design hazards analysis is completed.

\subsection{NPH AND EXTERNAL EVENTS}

The NPH and External events addressed by the hazard analysis for the ECRTS processes are provided in Table 1.

Table 1 - List of ECRTS NPH and External Events Considered in Hazards Analysis

\begin{tabular}{|c|c|c|c|}
\hline Seismic & Ash fall & Extreme Cold & $\begin{array}{c}\text { Explosion - vehicle fuel or } \\
\text { propane or welding gas }\end{array}$ \\
\hline Wind & Rain & $\begin{array}{c}\text { Loss of Electrical } \\
\text { Power }\end{array}$ & $\begin{array}{c}\text { Aircraft crash - below extremely } \\
\text { unlikely }\end{array}$ \\
\hline Flood & Lightning & Range Fire & Adjacent facility Fire \\
\hline Snow & Extreme heat & Vehicle Crash & Facility fire \\
\hline
\end{tabular}

The results of the NPH "What/If" hazards analysis are documented in Appendix C. 


\subsection{OPERATION SEQUENCES (GAP ANALYSIS)}

The operation sequence steps for Modes 1, $8 \& 9$ evaluated by the "What/ff" hazard analysis are provided in Appendix A. Included is the evaluation of differences resulting from the design progression from draft preliminary design operation sequence steps addressed in PRC-STP00382 versus the operation sequence steps addressed in this analysis.

The results of the Operation Sequences (Gap Analysis) "What/If" hazards analysis are documented in Appendix D.

\subsection{HAZARD ASSESSMENT TEAM}

The participants in Hazard Assessments for the draft preliminary design hazards analysis are shown in Appendix B. Following the release of the preliminary design information, (e.g. PFDs, P\&IDs, revised project documents) reviews were made to determine if changes to the results of the draft preliminary design hazard analysis results are necessary. It was determined that changes to the design affecting NPH and external event results were not significant enough to require additional meetings. The changes to the operation sequence steps were reviewed by representatives from Process Engineering and Nuclear Safety and were judged to not require additional meetings. The identified changes were distributed for project review prior to incorporation in this document.

\subsection{UNMITIGATED FREQUENCY AND CONSEQUENCE ESTIMATES}

Unmitigated radiological consequence and frequency levels were assigned to hazardous conditions identified by the "What/If" Hazard Analysis. The onsite and offsite radiological consequence levels are shown in Tables 2 and 3, respectively.

Table 2 - Collocated Worker and Offsite Public Consequence Levels*

\begin{tabular}{|l|c|c|}
\hline $\begin{array}{c}\text { Consequence } \\
\text { Level }\end{array}$ & $\begin{array}{c}\text { Collocated Worker (100 } \\
\text { m) }\end{array}$ & Offsite Public \\
\hline High & $>100 \mathrm{rem}$ & $>25 \mathrm{rem}$ \\
\hline Moderate & $\geq 25 \mathrm{rem}$ & $\geq 5 \mathrm{rem}$ \\
\hline Low & $<25 \mathrm{rem}$ & $<5 \mathrm{rem}$ \\
\hline
\end{tabular}

*Modified from PRC-STD-NS-8736, Table 2-1. Specifically, the moderate and low consequence level for the offsite public is set at 5 rem (versus $1 \mathrm{rem}$ ) based on guidance in DOE-STD-1189-2008. 
PRC-STP-00473 REV 0

Table 3 - Frequency Levels*

\begin{tabular}{|l|l|}
\hline \multicolumn{1}{|c|}{ Frequency Level } & \multicolumn{1}{c|}{ Frequency } \\
\hline Anticipated & Above $1 \mathrm{E}-2 / \mathrm{yr}$ \\
\hline Unlikely & $1 \mathrm{E}-4$ to $1 \mathrm{E}-2 / \mathrm{yr}$ \\
\hline Extremely Unlikely & $1 \mathrm{E}-6$ to $1 \mathrm{E}-4 / \mathrm{yr}$ \\
\hline Beyond Extremely Unlikely & Below $1 \mathrm{E}-6 / \mathrm{yr}$ \\
\hline
\end{tabular}

*Derived from PRC-STD-NS-8736, Table 2-2

In addition the Seismic Design Criteria and other NPH design criteria to be applied as required by DOE STD 1189-2008, and the Owendoff letter are shown in Table 4.

Table 4 - Guidance for SDC \& Other NPH Criteria Based on Unmitigated NPH Induced SSC Failures

\begin{tabular}{|c|c|c|c|}
\hline Seismic Category & $\begin{array}{c}\text { Other NPH } \\
\text { Category (1) }\end{array}$ & Collocated Worker & Public \\
\hline SDC-1 & PC-1 & Dose $<5$ rem & Not applicable (2) \\
\hline SDC-2 & PC-2 & $5 \mathrm{rem}<$ dose $<100 \mathrm{rem}$ & $5 \mathrm{rem}<$ Dose $<25 \mathrm{rem}$ \\
\hline SDC-3 & PC-3 & $<100$ rem $<$ dose & $25 \mathrm{rem}<$ dose \\
\hline \multicolumn{4}{|c|}{$\begin{array}{l}\text { (1) DOE Memorandum, April 15, 2009, Implementation of DOE-STD-1189, Integration of Safety into the } \\
\text { Design Process for Environmental Management Activities, J. M. Owendoff to Distribution, } \\
\text { U.S. Department of Energy, Washington, D.C.. } \\
\text { (2) A Hazard Category 1,2, or } 3 \text { nuclear facility with consequences to a collocated worker from failure of an } \\
\text { SSC in a seismic event will require that SSC to be classified as SDC-1 at a minimum. Therefore, a public } \\
\text { criterion for SDC-1 is not needed. }\end{array}$} \\
\hline
\end{tabular}

\subsection{RESULTS}

\subsection{IDENTIFIED ACCIDENT INITIATORS}

The Identified hazardous conditions for which prevention and/or mitigative SSCs or Administrative Controls may be required are identified in Appendix D. The specific hazardous conditions for the NPH or external events are provided in Table 5.

Table 5 - NPH and External Event Accident Initiators

\begin{tabular}{|l|c|c|c|c|c|}
\hline \multicolumn{1}{|c|}{ Event } & Spray Leak & $\begin{array}{c}\text { Splash and } \\
\text { splatter }\end{array}$ & $\begin{array}{c}\text { Hydrogen } \\
\text { Deflagration }\end{array}$ & Overpressure & $\begin{array}{c}\text { STSC } \\
\text { Overturn }\end{array}$ \\
\hline Seismic & $\mathrm{X}$ & $\mathrm{X}$ & $\mathrm{X}$ & $\mathrm{X}$ & $\mathrm{X}$ \\
\hline Wind & $\mathrm{X}$ & $\mathrm{X}$ & $\mathrm{X}$ & $\mathrm{X}$ & \\
\hline
\end{tabular}


PRC-STP-00473 REV 0

Table 5 - NPH and External Event Accident Initiators

\begin{tabular}{|c|c|c|c|c|c|}
\hline Event & Spray Leak & $\begin{array}{l}\text { Splash and } \\
\text { splatter }\end{array}$ & $\begin{array}{c}\text { Hydrogen } \\
\text { Deflagration }\end{array}$ & Overpressure & $\begin{array}{c}\text { STSC } \\
\text { Overturn }\end{array}$ \\
\hline Flood & & & $\mathrm{X}$ & $\mathrm{X}$ & \\
\hline Snow & $\mathrm{X}$ & $\mathrm{X}$ & $\mathrm{X}$ & $\mathrm{X}$ & \\
\hline Ashfall & $\mathrm{X}$ & $\mathrm{X}$ & $\mathrm{X}$ & $\mathrm{X}$ & \\
\hline Rain & & & $\mathrm{X}$ & $\mathrm{X}$ & \\
\hline Lightning & $\mathrm{X}$ & $\mathrm{X}$ & $\mathrm{X}$ & $\mathrm{X}$ & \\
\hline $\begin{array}{l}\text { Extreme heat } \\
\left(113^{\circ} \mathrm{F}\right)\end{array}$ & & & & $\mathrm{X}$ & \\
\hline $\begin{array}{l}\text { Low Ambient } \\
\text { Temperature } \\
\text { Extreme Cold } \\
\left(-23^{\circ} \mathrm{F}\right)\end{array}$ & $\mathrm{X}$ & $\mathrm{X}$ & $\mathrm{X}$ & $\mathrm{X}$ & \\
\hline $\begin{array}{l}\text { Loss of } \\
\text { Electrical Power } \\
\text { LOEP }\end{array}$ & $\mathrm{X}$ & $\mathrm{X}$ & $\mathrm{X}$ & $\mathrm{X}$ & \\
\hline Range Fire & $\mathrm{X}$ & $\mathrm{X}$ & $\mathrm{X}$ & $\mathrm{X}$ & $\mathrm{X}$ \\
\hline Vehicle Impact & $\mathrm{X}$ & $\mathrm{X}$ & $\mathrm{X}$ & $\mathrm{X}$ & $\mathrm{X}$ \\
\hline $\begin{array}{l}\text { Explosion - } \\
\text { vehicle fuel or } \\
\text { propane or } \\
\text { welding gas }\end{array}$ & $\mathrm{X}$ & $\mathrm{X}$ & $\mathrm{X}$ & $\mathrm{X}$ & $\mathrm{X}$ \\
\hline $\begin{array}{l}\text { Aircraft crash - } \\
\text { beyond } \\
\text { extremely } \\
\text { unlikely }\end{array}$ & $\mathrm{X}$ & $\mathrm{X}$ & $\mathrm{X}$ & $\mathrm{X}$ & $\mathrm{X}$ \\
\hline $\begin{array}{l}\text { Adjacent } \\
\text { facility Fire }\end{array}$ & $\mathrm{X}$ & $\mathrm{X}$ & $\mathrm{X}$ & $\mathrm{X}$ & $\mathrm{X}$ \\
\hline $\begin{array}{l}\text { Facility fire - } \\
\text { combustibles }\end{array}$ & $\mathrm{X}$ & $\mathrm{X}$ & $\mathrm{X}$ & $\mathrm{X}$ & $\mathrm{X}$ \\
\hline
\end{tabular}

\subsection{IDENTIFIED UNMITIGATED HAZARDOUS CONDITIONS}

The hazards analysis results shown in Appendices $\mathrm{C}$ and $\mathrm{D}$ indentified the following hazardous conditions as providing significant uncontrolled releases.

- Spray leak 
- Splash and splatter

- Deflagration

- Overpressure STS

- Overturn STSC, spill.

It is noted that the splash and splatter event includes a pool release component. The hazardous condition identified as "spread of contamination" encompasses a range of radiological conditions including fixed or low level airborne radioactivity that is a serious radiological concern but does not lead to a facility worker getting greater than 100 rem and would not likely be measurable for the collocated worker.

\subsection{FREQUENCY AND CONSEQUENCE LEVELS}

The frequency and consequence levels assigned to each hazardous condition are shown in Appendix E and are based on Table 6. The NPH initiated uncontrolled release is unlikely due to the initiating phenomena frequency. The unmitigated consequences are considered the same as events analyzed for the ECRTS conceptual safety design report. Similar consequences would result from the uncontrolled releases postulated as part of the gap analysis, but the frequency of those events is considered to be anticipated.

Table 6 - Basis for Appendix E Frequency and Consequence Levels

\begin{tabular}{|c|c|c|c|c|c|}
\hline \multirow{2}{*}{$\begin{array}{l}\text { Hazardous } \\
\text { Condition }\end{array}$} & \multirow{2}{*}{ Source Material $^{1}$} & \multirow{2}{*}{$\begin{array}{l}\text { Unmitigated } \\
\text { Frequency }\end{array}$} & \multicolumn{3}{|c|}{ Unmitigated Consequence } \\
\hline & & & $\begin{array}{l}\text { Facility } \\
\text { Worker }\end{array}$ & Onsite & Offsite \\
\hline \multirow{5}{*}{$\begin{array}{l}\text { Seismic induced } \\
\text { Spray Leak }\end{array}$} & Slurry (retrieval and transfer) & $\mathrm{U}$ & $\mathrm{H}$ & $\mathrm{H}$ & $\mathrm{L}$ \\
\hline & Slurry (overfill recovery) & $\mathrm{U}$ & $\mathrm{H}$ & $\mathrm{H}$ & $\mathrm{L}$ \\
\hline & Sand filter backwash & $\mathrm{U}$ & $\mathrm{L}$ & $\mathrm{L}$ & $\mathrm{L}$ \\
\hline & $\begin{array}{l}\text { Slurry (overfill recovery line } \\
\text { failure) }\end{array}$ & $\mathrm{U}$ & $\mathrm{H}$ & $\mathrm{H}$ & L \\
\hline & $\begin{array}{l}\text { Slurry (overfill recovery } \\
\text { diaphragm pump failure) }\end{array}$ & $\mathrm{U}$ & $\mathrm{L}$ & $\mathrm{L}$ & $\mathrm{L}$ \\
\hline \multirow{5}{*}{$\begin{array}{l}\text { Splash and } \\
\text { Splatter/Pool }\end{array}$} & Slurry (retrieval and transfer) & $\mathrm{U}$ & $\mathrm{L}$ & $\mathrm{L}^{*}$ & $\mathrm{~L}$ \\
\hline & STSC supernate & $\mathrm{U}$ & $\mathrm{L}$ & $\mathrm{L}$ & $\mathrm{L}$ \\
\hline & Sand filter backwash & $\mathrm{U}$ & $\mathrm{L}$ & $\mathrm{L}$ & $\mathrm{L}$ \\
\hline & $\begin{array}{l}\text { Slurry (overfill recovery line } \\
\text { failure) }\end{array}$ & $\mathrm{U}$ & $\mathrm{L}$ & $\mathrm{L}$ & $\mathrm{L}$ \\
\hline & $\begin{array}{l}\text { Slurry (overfill recovery } \\
\text { diaphragm pump failure) }\end{array}$ & $\mathrm{U}$ & $\mathrm{L}$ & $\mathrm{L}$ & $\mathrm{L}$ \\
\hline
\end{tabular}


PRC-STP-00473 REV 0

Table 6 - Basis for Appendix E Frequency and Consequence Levels

\begin{tabular}{|c|c|c|c|c|c|}
\hline \multirow{2}{*}{$\begin{array}{l}\text { Hazardous } \\
\text { Condition }\end{array}$} & \multirow{2}{*}{ Source Material ${ }^{1}$} & \multirow{2}{*}{$\begin{array}{l}\text { Unmitigated } \\
\text { Frequency }\end{array}$} & \multicolumn{3}{|c|}{ Unmitigated Consequence } \\
\hline & & & $\begin{array}{l}\text { Facility } \\
\text { Worker }\end{array}$ & Onsite & Offsite \\
\hline \multirow{5}{*}{$\begin{array}{l}\text { Hydrogen } \\
\text { Deflagration }\end{array}$} & STSC & U & $\mathrm{H}^{2}$ & $\mathrm{~L}$ & $\mathrm{~L}$ \\
\hline & Transfer Line Service Box & $\mathrm{U}$ & $\mathrm{H}^{2}$ & $\mathrm{~L}$ & $\mathrm{~L}$ \\
\hline & Decant Pump Box ${ }^{3}$ & $\mathrm{U}$ & $\mathrm{H}^{2}$ & $\mathrm{~L}$ & $\mathrm{~L}$ \\
\hline & Sand Filter ${ }^{3}$ & $\mathrm{U}$ & $\mathrm{H}^{2}$ & $\mathrm{~L}$ & $\mathrm{~L}$ \\
\hline & Sand Filter Enclosure ${ }^{3}$ & $\mathrm{U}$ & $\mathrm{H}^{2}$ & $\mathrm{~L}$ & $\mathrm{~L}$ \\
\hline Overpressurization & Sludge slurry & $\mathrm{U}$ & $\mathrm{L}$ & $\mathrm{L}$ & $\mathrm{L}$ \\
\hline \multicolumn{6}{|c|}{$\begin{array}{l}\text { 'Consequences based on settler tank sludge which has a higher radionuclide inventory than } \mathrm{KE} \text { or } \mathrm{KW} \text { Basin sludge. } \\
\text { For a given hazardous condition (e.g., spray release) the consequences vary as a function of the estimated volume } \\
\text { percent solids. }\end{array}$} \\
\hline \multicolumn{6}{|c|}{$\begin{array}{l}{ }^{2} \mathrm{~A} \text { high consequence is assigned to the facility worker for hydrogen deflagrations based on the potential for prompt } \\
\text { death or serious injury. }\end{array}$} \\
\hline \multicolumn{6}{|c|}{$\begin{array}{l}{ }^{3} \text { PRC-STP-CN-N-00401 does not analyze hydrogen deflagrations in the Decant Pump Box, sand filter, or Sand Filter } \\
\text { Enclosure. The low consequences are qualitatively assigned based on the low consequences calculated for } \\
\text { deflagrations in the STSC and Transfer Line Service Box. }\end{array}$} \\
\hline $\begin{array}{l}\mathrm{A}=\text { Anticipated } \\
\mathrm{H}=\text { High } \\
\mathrm{M}=\text { Moderate } \\
\mathrm{L}=\text { Low } \\
\mathrm{L}^{*}=\text { Low }, \text { but requ }\end{array}$ & SDC-2 and Other NPH PC-2 & riteria see $\mathrm{Tab}$ & $4(5 \mathrm{rem}$ & 10 & \\
\hline
\end{tabular}

\subsection{REFERENCES}

DOE Memorandum, April 15, 2009, Implementation of DOE-STD-1189, Integration of Safety into the Design Process for Environmental Management Activities, J. M. Owendoff to Distribution, U.S. Department of Energy, Washington, D.C.

HNF-34374, 2011, Sludge Treatment Project Safety Design Strategy, Rev 4, CH2M HILL Plateau Remediation Company, Richland, Washington

HNF-41051, 2011, Preliminary STP Container and Settler Sludge Process System Description and Material Balance, Rev. 7, CH2M HILL Plateau Remediation Company, Richland, Washington.

PRC-STD-NS-8736, 2011, CHPRC Safety Analysis and Risk Assessment Handbook (SARAH), Rev 0 Chg 0, CH2M HILL Plateau Remediation Company, Richland, Washington. 
PRC-STP-CN-00401, 2011, Sludge Treatment Project Engineered Container Retrieval and Transfer System Draft Preliminary Design Accident Analysis ", Rev 1, CH2M HILL Plateau Remediation Company, Richland, Washington.

HNF-SD-SNF-TI-015, 2010, Spent Nuclear Fuel Project Technical Databook, Volume 2, Sludge, Rev. 14B, CH2M HILL Plateau Remediation Company, Richland, Washington.

HNF-SD-SNF-TI-015, 2010, Spent Nuclear Fuel Technical Databook, Volume 3, K Basin Closure Project Technical Databook KOP Material (OCRWM), Rev 1, CH2M HILL Plateau Remediation Company, Richland, Washington

PRC-STP-00054, 2011, Preliminary STP Container and Settler Sludge Process Control Plan, Rev 2, CH2M HILL Plateau Remediation Company, Richland, Washington.

PRC-STP-00124, 2009, Sludge Treatment Project Engineered Container Retrieval and Transfer System Conceptual Design Hazard Analysis, Rev 1, CH2M HILL Plateau Remediation Company, Richland, Washington.

PRC-STP-00346, 2010, Sludge Treatment Project Engineered Container Retrieval and Transfer System Draft Preliminary Design Hazard And Operability Study, Rev 0, CH2M HILL Plateau Remediation Company, Richland, Washington.

PRC-STP-00358, 2011, Sludge Treatment Project Engineered Container Retrieval and Transfer System Conceptual Design Hazard Analysis, Rev 2, CH2M HILL Plateau Remediation Company, Richland, Washington.

PRC-STP-00382, 2011, Sludge Treatment Project Engineered Container Retrieval And Transfer System Draft Preliminary Design Hazard Analysis Supplement 1. Rev 0 \& 0A, CH2M HILL Plateau Remediation Company, Richland, Washington.

PRC-STP-00409, 2011, Sludge Treatment Project Engineered Container Retrieval and Transfer System Draft Preliminary Design Control Decision, Rev 0, CH2M HILL Plateau Remediation Company, Richland, Washington.

PRC-STP-00467, 2011, "Sludge Treatment Project Engineered Container Retrieval and Transfer System Preliminary Design Hazard And Operability Study" Rev 0, CH2M HILL Plateau Remediation Company, Richland, Washington. 
PRC-STP-00473 REV 0

APPENDIX A

OPERATION SEQUENCE STEPS

A- i 
PRC-STP-00473 REV 0

MODE 1 - RECEIVE STS TRAILER \& CONNECT STSC

\begin{tabular}{|c|c|c|c|c|c|c|c|}
\hline Mode & Step & $\begin{array}{l}\text { Current Operations Sequence Description } \\
\text { (from PRC-STP-00358 Rev 1) }\end{array}$ & $\begin{array}{l}\text { Step } \\
\text { Change }\end{array}$ & $\begin{array}{l}\text { Content } \\
\text { Change }\end{array}$ & $\begin{array}{l}\text { Discussion } \\
\text { (basis for change) }\end{array}$ & & $\begin{array}{l}\text { Previous Step \& Description } \\
\text { (PRC-STP-00382) }\end{array}$ \\
\hline 1 & 1 & Enter Annex and open loading bay door & $\mathrm{N}$ & $\mathrm{N}$ & & 1 & Enter Annex and open loading bay door \\
\hline 1 & 2 & Position and align STS trailer on weigh scales & $\mathrm{N}$ & $\mathrm{N}$ & & 2 & Position and align STS trailer on weigh scales \\
\hline 1 & 3 & Secure STS trailer & $\mathrm{N}$ & $\mathrm{N}$ & & 3 & Secure STS trailer \\
\hline 1 & 4 & Disconnect STS tractor and exit loading bay ramp & $\mathrm{N}$ & $\mathrm{N}$ & & 4 & Disconnect STS tractor and exit loading bay ramp \\
\hline 1 & 5 & Close Annex loading bay door & $\mathrm{N}$ & $\mathrm{N}$ & & 5 & Close Annex loading bay door \\
\hline 1 & 6 & $\begin{array}{l}\text { Record trailer weight (WIT-740-401) and verify shipping } \\
\text { manifest. }\end{array}$ & $\mathrm{N}$ & $\mathrm{N}$ & & 6 & Weigh trailer with cask and record weight. \\
\hline 1 & 7 & Remove IP-2 bag from Drain Port & NA & New & \multirow{6}{*}{$\begin{array}{l}\text { Content changed because } \\
\text { of STS Cask purge } \\
\text { through drain port rather } \\
\text { than vent port and adding } \\
\text { a new hose (H-603) from } \\
\text { drain port to new drain } \\
\text { tank. }\end{array}$} & \multirow{6}{*}{\multicolumn{2}{|c|}{ 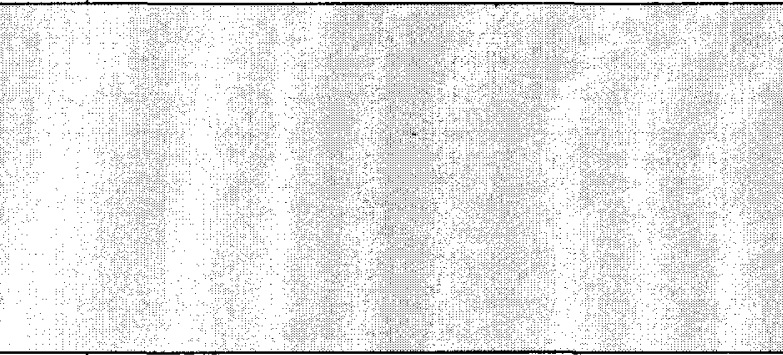 }} \\
\hline 1 & 8 & Remove Cask drain port cap and install drain port tool & NA & New & & & \\
\hline 1 & 9 & $\begin{array}{l}\text { Attach Purge Line H-602 to drain port tool. Align V-609 to } \\
\text { "Cask" position. Verify V-607 and V-608 are closed. }\end{array}$ & NA & New & & & \\
\hline 1 & 10 & Attach drain line $\mathrm{H}-603$ to drain port tool & NA & New & & & \\
\hline 1 & 11 & Open drain port & NA & New & & & \\
\hline 1 & 12 & Verify STS cask pressure is 0 psig (PI-760-606) & NA & New & & & \\
\hline 1 & 13 & Unzip/remove Outer IP-2 bag & $\mathrm{Y}$ & $\begin{array}{c}\text { No - was } \\
7 \\
\end{array}$ & & 7 & Unzip/remove Outer IP-2 bag. \\
\hline 1 & 14 & Unzip/remove Inner IP-2 bag & $\mathrm{Y}$ & $\begin{array}{c}\text { No- was } \\
8 \\
\end{array}$ & & 8 & Unzip/remove Inner IP-2 bag \\
\hline 1 & 15 & Install Lid Lift Fixture on STS cask lid ( 3 bolts) & $\mathrm{Y}$ & $\begin{array}{c}\text { No - was } \\
16\end{array}$ & & 16 & Install Lid Lift Fixture on STS cask lid \\
\hline 1 & 16 & Remove the STS Lid bolts ( 24 bolts) & $Y$ & $\begin{array}{c}\text { No - was } \\
17\end{array}$ & & 17 & Remove the STS Lid bolts \\
\hline 1 & 17 & $\begin{array}{l}\text { Remove the STS Lid and install onto the Lid Inspection } \\
\text { Station. }\end{array}$ & $\mathrm{Y}$ & $\begin{array}{l}\text { No - was } \\
18\end{array}$ & & 18 & $\begin{array}{l}\text { Remove the STS Lid and install onto the Lid Inspection } \\
\text { Station. }\end{array}$ \\
\hline 1 & 18 & $\begin{array}{l}\text { Verify V-103 and V-109 are OPEN. Verify V-101, AOV-104, } \\
\text { and AOV-105 are CLOSED. }\end{array}$ & $\mathrm{Y}$ & $\begin{array}{c}Y-\text { was } \\
19\end{array}$ & $\begin{array}{l}\text { AOV-104 changed to } \\
\text { closed and AOV-105 } \\
\text { verified closed } \\
\text { (corrections to current } \\
\text { configuration) }\end{array}$ & 19 & $\begin{array}{l}\text { Verify V-103, V-109 and AOV-104 are OPEN. Verify V- } \\
101 \text { is CLOSED }\end{array}$ \\
\hline 1 & 19 & Remove and store the 3-bolt blind flange from STSC Nozzle A. & $\mathrm{Y}$ & $\begin{array}{c}\text { No- was } \\
20\end{array}$ & & 20 & $\begin{array}{l}\text { Remove and store the 3-bolt blind flange from STSC Nozzle } \\
\text { A. }\end{array}$ \\
\hline 1 & 20 & $\begin{array}{l}\text { Remove contamination controls and end cap from Sludge line } \\
\text { connector. Air flows from the room into the TLSB through H- } \\
107 \text { and } \mathrm{H}-107 \text { annulus. IL-125 turns ON. }\end{array}$ & Y & $\begin{array}{l}Y-\text { was } \\
21\end{array}$ & Added IL- 125 status & 21 & $\begin{array}{l}\text { Remove contamination controls and end cap from Sludge } \\
\text { line connector. Air flows from the room into the TLSB } \\
\text { through H-107 and H-107 annulus }\end{array}$ \\
\hline
\end{tabular}


PRC-STP-00473 REV 0

MODE 1 - RECEIVE STS TRAILER \& CONNECT STSC

\begin{tabular}{|c|c|c|c|c|c|c|c|}
\hline Mode & Step & $\begin{array}{l}\text { Current Operations Sequence Description } \\
\text { (from PRC-STP-00358 Rev 1) }\end{array}$ & $\begin{array}{c}\text { Step } \\
\text { Change }\end{array}$ & $\begin{array}{l}\text { Content } \\
\text { Change }\end{array}$ & $\begin{array}{l}\text { Discussion } \\
\text { (basis for change) }\end{array}$ & & $\begin{array}{l}\text { Previous Step \& Description } \\
\text { (PRC-STP-00382) }\end{array}$ \\
\hline 1 & 21 & $\begin{array}{l}\text { Connect the Sludge Inlet Riverbend connector (Hose } \mathrm{H}-107) \text { to } \\
\text { the } 3 \text { bolt PUP connector on Nozzle A and torque the acom } \\
\text { nuts. Verify IL-118 is ON. }\end{array}$ & $\mathrm{Y}$ & $\begin{array}{l}\text { No - was } \\
22\end{array}$ & & 22 & $\begin{array}{l}\text { Connect the Sludge Inlet Riverbend connector (Hose } \mathrm{H}-107 \text { ) } \\
\text { to the } 3 \text { bolt PUP connector on Nozzle A and torque the } \\
\text { acorn nuts. Verify IL-118 is ON }\end{array}$ \\
\hline 1 & 22 & $\begin{array}{l}\text { Verify V-205 and V-201 are OPEN. Verify V-202 and V-206 } \\
\text { is CLOSED. }\end{array}$ & $\mathrm{Y}$ & $\begin{array}{l}Y \text { - was } \\
23\end{array}$ & $\begin{array}{l}\text { Added closed verification } \\
\text { for V-206 (verifies IXM } \\
\text { Water shutoff). }\end{array}$ & 23 & $\begin{array}{l}\text { Verify V-205 and V-201 are OPEN. Verify V-202 is } \\
\text { CLOSED }\end{array}$ \\
\hline 1 & 23 & Remove and store the 4-bolt blind flange from STSC Nozzle B. & $\mathrm{Y}$ & $\begin{array}{l}\text { No-was } \\
24\end{array}$ & & 24 & $\begin{array}{l}\text { Remove and store the 4-bolt blind flange from STSC Nozzle } \\
\text { B. }\end{array}$ \\
\hline 1 & 24 & $\begin{array}{l}\text { Remove contamination controls and end cap from Decant line } \\
\text { connector. Air flows from the room into the decant box } \\
\text { through } \mathrm{H}-203 \text { and } \mathrm{H}-203 \text { annulus. IL- } 223 \text { turns ON. }\end{array}$ & $\mathrm{Y}$ & $\begin{array}{l}\text { Y - was } \\
25\end{array}$ & $\begin{array}{l}\text { Added IL-223 status (FE } \\
720-202 \text { added) }\end{array}$ & 25 & $\begin{array}{l}\text { Remove contamination controls and end cap from } \\
\text { Decant/backwash line connector. Air flows from the room } \\
\text { into the decant box through } \mathrm{H}-203 \text { and } \mathrm{H}-203 \text { annulus }\end{array}$ \\
\hline 1 & 25 & $\begin{array}{l}\text { Connect the Decant Outlet/Backflush Inlet Riverbend } \\
\text { connector (Hose H-203) to the } 4 \text { bolt PUP connector on Nozzle } \\
\text { B and torque the acorn nuts. Verify IL-218 is ON. }\end{array}$ & $\mathrm{Y}$ & $\begin{array}{l}\text { No- was } \\
26\end{array}$ & & 26 & $\begin{array}{l}\text { Connect the Decant Outlet/Backflush Inlet Riverbend } \\
\text { connector (Hose H-203) to the } 4 \text { bolt PUP connector on } \\
\text { Nozzle B and torque the acom nuts. Verify IL- } 218 \text { is ON }\end{array}$ \\
\hline 1 & 26 & $\begin{array}{l}\text { Remove and store the } 2 \text { inch Camlok cap from Nozzle F2 and } \\
\text { connect the IXM Water Inlet onto the } 2 \text { inch Camlok } \\
\text { connection (Hose H-752). }\end{array}$ & $\mathrm{Y}$ & $\begin{array}{l}\text { No - was } \\
27\end{array}$ & & 27 & $\begin{array}{l}\text { Remove and store the } 2 \text { inch Camlok cap from Nozzle F2 } \\
\text { and connect the IXM Water Inlet onto the } 2 \text { inch Camlok } \\
\text { connection (Hose H-752) }\end{array}$ \\
\hline 1 & 27 & $\begin{array}{l}\text { Remove and store the } 4 \text { inch Camlok cap from Nozzle F1 and } \\
\text { connect the } 4 \text { inch STSC Vent Camlok connection (Hose H- } \\
501 \text { ). }\end{array}$ & $\mathrm{Y}$ & $\begin{array}{l}\text { No - was } \\
28\end{array}$ & & 28 & $\begin{array}{l}\text { Remove and store the } 4 \text { inch Camlok cap from Nozzle F1 } \\
\text { and connect the } 4 \text { inch STSC Vent Camlok connection (Hose } \\
\text { H-501) }\end{array}$ \\
\hline 1 & 28 & $\begin{array}{l}\text { Connect the Purge Inlet } 1 \text { inch Quick Connect (Hose H-604) to } \\
\text { Nozzle S2. }\end{array}$ & $\mathrm{Y}$ & $\begin{array}{c}Y \text { - was } \\
29\end{array}$ & $\begin{array}{l}\text { Identified Nozzle } \\
\text { designator (S2) }\end{array}$ & 29 & Connect the Purge Inlet 1 inch Quick Connect (Hose H-604) \\
\hline 1 & 29 & $\begin{array}{l}\text { Connect I\&C connector to LDE-710-103 (Leak Detector) and } \\
\text { LE-740-401 (Level Detector) Safety Significant instruments }\end{array}$ & $\mathrm{Y}$ & $\begin{array}{l}\text { No - was } \\
30\end{array}$ & & 30 & $\begin{array}{l}\text { Connect I\&C connector to LDE-710-103 (Leak Detector) } \\
\text { and LE-740-401 (Level Detector) Safety Significant } \\
\text { instruments Connect STSC instruments }\end{array}$ \\
\hline 1 & 30 & $\begin{array}{l}\text { Connect I\&C connector to LDE-720-204 (Leak Detector) and } \\
\text { LSH-740-402 (Level Switch) General Service instruments }\end{array}$ & $\mathrm{Y}$ & $\begin{array}{c}Y \text { - was } \\
31\end{array}$ & $\begin{array}{l}\text { Corrected LDE-720-410 } \\
\text { to LDE-720-204 }\end{array}$ & 31 & $\begin{array}{l}\text { Connect I\&C connector to LDE-720-410 (Leak Detector) } \\
\text { and LSH-740-402 (Level Switch) General Service } \\
\text { instruments }\end{array}$ \\
\hline 1 & 31 & $\begin{array}{l}\text { Turn on STSC Instrument disconnect switch. Verify instrument } \\
\text { Health is OK (IL- } 408 \text { and IL- } 412 \text { are ON) }\end{array}$ & NA & New & & & \\
\hline 1 & 32 & $\begin{array}{l}\text { Weigh and record initial filling condition weight (WIT-740- } \\
\text { 401). }\end{array}$ & $\mathrm{N}$ & $\mathrm{Y}$ & Identified instrument & 32 & Weigh and record initial filling condition weight \\
\hline 1 & 33 & $\begin{array}{l}\text { Select/Verify sludge type on PNL-401 (ECRT-KS-401 to 'K- } \\
\text { East', 'K-West', or 'Settler' position) and reset PNL-401: Press } \\
\text { HS-402 'SIP RESET' pushbutton. }\end{array}$ & $\mathrm{N}$ & $\mathrm{N}$ & & 33 & $\begin{array}{l}\text { Select Sludge Type on PNL-401 (ECRT-KS-401 to 'K-East', } \\
\text { 'KWest', or 'Settler' position) and reset PNL-401: Press HS- } \\
402 \text { 'reset' pushbutton }\end{array}$ \\
\hline
\end{tabular}


PRC-STP-00473 REV 0

MODE 1 - RECEIVE STS TRAILER \& CONNECT STSC

\begin{tabular}{|c|c|c|c|c|c|c|c|}
\hline Mode & Step & $\begin{array}{l}\text { Current Operations Sequence Description } \\
\text { (from PRC-STP-00358 Rev 1) }\end{array}$ & $\begin{array}{l}\text { Step } \\
\text { Change }\end{array}$ & $\begin{array}{l}\text { Content } \\
\text { Change }\end{array}$ & $\begin{array}{l}\text { Discussion } \\
\text { (basis for change) }\end{array}$ & & $\begin{array}{l}\text { Previous Step \& Description } \\
\text { (PRC-STP-00382) }\end{array}$ \\
\hline 1 & 34 & $\begin{array}{l}\text { Initiate operability test of LSH-740-402: Verify ECRT-HS- } \\
113 \text { in 'DISABLE' position. }\end{array}$ & $\mathrm{N}$ & $\mathrm{Y}$ & $\begin{array}{l}\text { Corrected LSH-740-313 } \\
\text { to LDE-740-402, added } \\
\text { verification for disabling } \\
\text { of flush, deleted HS-701 }\end{array}$ & 34 & $\begin{array}{l}\text { Initiate verifying operability of LSH-740-313: Place ECRT- } \\
\text { HS-701 in 'test' position. }\end{array}$ \\
\hline 1 & 35 & Record starting value of water meter FQI-770-701 & $\mathrm{N}$ & $\mathrm{N}$ & & 35 & Record starting value of water meter FQI-770-701 \\
\hline 1 & 36 & $\begin{array}{l}\text { Open Valve STP-V- } 731 \text { and begin initial fill of STSC with } \\
\text { IXM water. }\end{array}$ & $\mathrm{N}$ & $\mathrm{N}$ & & 36 & $\begin{array}{l}\text { Open Valve STP-V-731 and begin initial fill of STSC with } \\
\text { IXM water }\end{array}$ \\
\hline 1 & 37 & Close V-109 and V-205 & NA & New & $\begin{array}{l}\text { Shut off TLSB and DPB } \\
\text { air vents. }\end{array}$ & & $x^{2}+x_{2}$ \\
\hline 1 & 38 & $\begin{array}{l}\text { Level reaches LSH-740-402 level. IL-13 and IL-402 turn ON. } \\
\text { Interlock I-3 trips and shuts off IXM water supply valve AOV- } \\
701 \text {, Bladder flush valve AOV-102 opens. HS-113 'DISABLE' } \\
\text { mode prevents bladder flush from occurring (AOV-113 held } \\
\text { closed). }\end{array}$ & $\mathrm{Y}$ & $\begin{array}{l}\text { No - was } \\
37\end{array}$ & $\begin{array}{l}\text { Corrected LSH-740-713 } \\
\text { to LSH-740-402, changed } \\
\text { AOV-102 from closed to } \\
\text { open, added HS-113 } \\
\text { DISABLE mode to hold } \\
\text { new valve AOV-113 } \\
\text { closed. }\end{array}$ & 37 & $\begin{array}{l}\text { Level reaches LSH-740-713 level. IL-13 and IL- } 402 \text { turn } \\
\text { ON. Interlock I-3 trips and shuts off IXM water supply valve } \\
\text { AOV-701. HS-701 'test' mode prevents bladder flush from } \\
\text { occurring (AOV-102 held closed). }\end{array}$ \\
\hline 1 & 39 & Close valve STP-V-731 when indicator reflects full STSC. & Y & $\begin{array}{l}\text { No - was } \\
38\end{array}$ & & 38 & Secure Valve STP-V-731 when indicator reflects full STSC \\
\hline 1 & 40 & Record ending value of water meter FQI-770-701 & $\mathrm{Y}$ & $\begin{array}{c}\text { No - was } \\
39\end{array}$ & & 39 & Record ending value of water meter FQI-770-701 \\
\hline 1 & 41 & $\begin{array}{l}\text { Drain (V-736) and disconnect IXM water hose } \mathrm{H}-752 \text { from } \\
\text { Nozzle F2 }\end{array}$ & $\mathrm{Y}$ & $\begin{array}{l}Y \text { - was } \\
40\end{array}$ & $\begin{array}{l}\text { Added drain (new valve } \\
\text { V-736) }\end{array}$ & 40 & Disconnect the IXM Water (H-752) from Nozzle F2 \\
\hline 1 & 42 & $\begin{array}{l}\text { Connect the Purge Outlet } 1 \text { inch Quick Connect (Hose H-659) } \\
\text { to Nozzle F2. }\end{array}$ & $\mathrm{Y}$ & $\begin{array}{c}Y \text { - was } \\
41\end{array}$ & Change 2 inch to 1 inch & 41 & $\begin{array}{l}\text { Connect the Purge Outlet } 2 \text { inch Quick Connect (Hose H- } \\
659 \text { ) to Nozzle F2 }\end{array}$ \\
\hline 1 & 43 & $\begin{array}{l}\text { Verify pressure gauge PI-760-652 is reading at least } 1 \text { inch of } \\
\text { water vacuum. }\end{array}$ & $\mathrm{Y}$ & $\begin{array}{l}\text { No - was } \\
42\end{array}$ & . & 42 & $\begin{array}{l}\text { Verify pressure gauge PI-760-652 is reading at least } 1 \text { inch } \\
\text { of water vacuum }\end{array}$ \\
\hline 1 & 44 & $\begin{array}{l}\text { Record first STSC tare weight and level W1 and L1 (WIT-740- } \\
401 \text { and LIT-740-401). Initialize PNL-401 by pressing 'high } \\
\text { tare' pushbutton HS-403. (Safety Significant Measurement) }\end{array}$ & $\mathrm{Y}$ & $\begin{array}{c}Y-\text { was } \\
43\end{array}$ & $\begin{array}{l}\text { Added Instrument } \\
\text { designation and safety } \\
\text { significant measurement } \\
\text { designation. } \\
\end{array}$ & 43 & $\begin{array}{l}\text { Record first STSC tare weight and level W1 and L1. } \\
\text { Initialize PNL-401 by pressing 'high tare' pushbutton HS- } \\
403 \text {. }\end{array}$ \\
\hline 1 & 45 & $\begin{array}{l}\text { Verify AOV-201 is in 'filter' position (HS-201 'filter') Verify } \\
\text { IL-215 indicates 'filter.' Open V-202 }\end{array}$ & $\mathrm{Y}$ & $\mathrm{Y}$ - was 44 & \begin{tabular}{|l|} 
Corrected IL-201 to IL- \\
215, Added open V-202 \\
\end{tabular} & 44 & $\begin{array}{l}\text { Verify AOV-201 is in 'filter' position (HS-201 'filter') Verify } \\
\text { IL-201 indicates 'filter' }\end{array}$ \\
\hline 1 & 46 & $\begin{array}{l}\text { Start decant pump P-201 by opening AOV-760 (HS-760 'start') } \\
\text { Verify IL-760 indicates 'running' }\end{array}$ & $\mathrm{Y}$ & $\begin{array}{l}\text { No - was } \\
45\end{array}$ & HS-701 deleted. & 45 & $\begin{array}{l}\text { Start decant pump P-201 by opening AOV-760 (HS-760 } \\
\text { 'start') Verify IL-761 indicates 'running' }\end{array}$ \\
\hline 1 & 47 & $\begin{array}{l}\text { STSC level drops, resetting interlock I-3. Verify IL-13 and IL- } \\
402 \text { turn OFF. }\end{array}$ & $\mathrm{Y}$ & $\begin{array}{l}\text { No - was } \\
46\end{array}$ & & 46 & $\begin{array}{l}\text { STSC level drops, resetting interlock I-3. Verify IL-13 and } \\
\text { IL-402 tum OFF }\end{array}$ \\
\hline
\end{tabular}


PRC-STP-00473 REV 0

MODE 1 - RECEIVE STS TRAILER \& CONNECT STSC

\begin{tabular}{|c|c|c|c|c|c|c|c|}
\hline Mode & Step & $\begin{array}{l}\text { Current Operations Sequence Description } \\
\text { (from PRC-STP-00358 Rev 1) }\end{array}$ & $\begin{array}{l}\text { Step } \\
\text { Change }\end{array}$ & $\begin{array}{l}\text { Content } \\
\text { Change }\end{array}$ & $\begin{array}{l}\text { Discussion } \\
\text { (basis for change) }\end{array}$ & & $\begin{array}{l}\text { Previous Step \& Description } \\
\text { (PRC-STP-00382) }\end{array}$ \\
\hline 1 & 48 & $\begin{array}{l}\text { Pump until loss of suction. Turbidity meter Al-720-202 trips } \\
\text { Interlock I-2 (ASH-720-202) and shuts off P-201 (AOV-760 } \\
\text { closes) Verify IL-761 indicates 'stopped' }\end{array}$ & $\mathrm{Y}$ & $\begin{array}{c}Y \text { - was } \\
47\end{array}$ & $\begin{array}{l}\text { Change to reflect new } \\
\text { Turbidity meter AI-720- } \\
202 \text { trips and Interlock I- } \\
2 \text { and deletion of ASH- } \\
720-20 \text {. }\end{array}$ & 47 & $\begin{array}{l}\text { Pump until loss of suction. Turbidity meter Al-720-201 trips } \\
\text { Interlock I-2 (ASH-720-201) and shuts off P-201 (AOV-760 } \\
\text { closes) Verify IL-760 indicates 'stopped' }\end{array}$ \\
\hline 1 & 49 & $\begin{array}{l}\text { Record second STSC tare weight and level W2 and L2 (WIT- } \\
740-401 \text { and LIT-740-401). Initialize PNL-401 by pressing } \\
\text { 'low tare' pushbutton HS- } 406 \text {. Calculate and verify STSC } \\
\text { geometry factor. (Safety Significant Measurement) }\end{array}$ & $\mathrm{Y}$ & Y- was 48 & $\begin{array}{l}\text { Added Instrument } \\
\text { designation and safety } \\
\text { significant measurement } \\
\text { designation. }\end{array}$ & 48 & $\begin{array}{l}\text { Record second STSC tare weight and level W2 and L2. } \\
\text { Initialize PNL-401 by pressing 'low tare' pushbutton HS- } 406 \text {. } \\
\text { Calculate and verify STSC geometry factor. }\end{array}$ \\
\hline 1 & 50 & Secure from test mode: & $\mathrm{Y}$ & $\begin{array}{c}Y \text { - was } \\
49 \\
\end{array}$ & $\begin{array}{l}\text { Deleted HS -701 in test } \\
\text { mode. }\end{array}$ & 49 & Secure from 'test' mode (HS-701 to 'auto' position) \\
\hline 1 & 51 & $\begin{array}{l}\text { Close V-109. Open V-101 and visually verify alignment of all } \\
\text { manual valves in TLSB (V-101, V-103 OPEN, V-102 in } \\
\text { 'transfer' position, all others closed). Verify IL- } 125 \text { is OFF. }\end{array}$ & $\mathrm{N}$ & $\begin{array}{l}Y-\text { was } \\
50\end{array}$ & $\begin{array}{l}\text { Deleted close AOV-104 } \\
\text { (closed in Step 18). } \\
\text { Added IL-125 status. }\end{array}$ & 50 & $\begin{array}{l}\text { Close V-109 and AOV-104. Open V-101 and visually verify } \\
\text { alignment of all manual valves in TLSB (V-101, V-102, V- } \\
103 \text { open, all others closed) }\end{array}$ \\
\hline 1 & 52 & $\begin{array}{l}\text { Close V-205. Open V-202, and visually verify alignment of all } \\
\text { manual valves in decant box (V-201, V-202, V-204, V-210, V- } \\
227 \text { OPEN, all others closed). IL-223 is OFF. }\end{array}$ & $\mathrm{Y}$ & $\begin{array}{c}Y-\text { was } \\
51\end{array}$ & Added IL-223 status. & 51 & $\begin{array}{l}\text { Close V-205. Open V-202, and visually verify alignment of } \\
\text { all manual valves in decant box (V-201, V-202, V-204, V- } \\
210, \mathrm{~V}-227 \text { OPEN, all others closed) }\end{array}$ \\
\hline 1 & 53 & Exit and secure Annex loading bay. & $\mathrm{Y}$ & $\begin{array}{c}\text { No - was } \\
52 \\
\end{array}$ & & 52 & Exit and secure Annex loading bay \\
\hline
\end{tabular}


PRC-STP-00473 REV 0

MODE 8 - DISCONNECT \& PURGE STSC

\begin{tabular}{|c|c|c|c|c|c|c|c|}
\hline Mode & Step & $\begin{array}{l}\text { Current Operations Sequence Description } \\
\text { (from PRC-STP-00358 Rev 1) }\end{array}$ & \begin{tabular}{|c|} 
Step \\
Change \\
\end{tabular} & $\begin{array}{l}\text { Content } \\
\text { Change }\end{array}$ & $\begin{array}{c}\text { Discussion } \\
\text { (basis for change) }\end{array}$ & & $\begin{array}{c}\text { Previous Step \& Description } \\
\text { (PRC-STP-00382) }\end{array}$ \\
\hline 8 & 1 & Enter Annex loading bay & $\mathrm{N}$ & $\mathrm{N}$ & & 1 & Enter Annex loading bay \\
\hline 8 & 2 & Perform final LXM flush of sludge line: Close V-101, open AOV-104 & $\mathrm{N}$ & Y & Deleted V-712 & 2 & $\begin{array}{l}\text { Perform final IXM flush of sludge line: Close V-101, open V- } \\
712 \text { and AOV-104 }\end{array}$ \\
\hline 8 & 3 & Secure from final sludge line flush: Close AOV-104 & $\mathrm{N}$ & $\mathrm{Y}$ & Deleted V-712 & 3 & Secure from final sludge line flush: Close AOV-104 and V-712 \\
\hline 8 & 4 & Drain sludge line: Open V-109 & $\mathrm{N}$ & $\mathrm{Y}$ & $\begin{array}{l}\text { Deleted open AOV-104 } \\
\text { (valve line up changed). }\end{array}$ & 4 & Drain sludge line: Open V-109 and AOV-104 \\
\hline 8 & 5 & Perform final Decant line flush: Close V-202, Open V-206 & $\mathrm{N}$ & $\mathrm{N}$ & & 5 & $\begin{array}{l}\text { Perform final Decant/backwash line flush: Close V-202, Open } \\
\text { V-206 }\end{array}$ \\
\hline 8 & 6 & Top off STSC with IXM water to final level for shipping using V-206 & Y & No - was 7 & Corrected sequence & 7 & $\begin{array}{l}\text { Top off STSC with IXM water to final level for shipping using } \\
\text { V-206 }\end{array}$ \\
\hline 8 & 78 & Secure from final Decant line flush: Close V-206 & $\mathrm{Y}$ & No - was 6 & Corrected sequence & 6 & Secure from final Decant/backwash line flush: Close V-206 \\
\hline 8 & 8 & Drain decant line: Open V-205 & $\mathrm{N}$ & $\mathrm{N}$ & & 8 & Drain decant/backwash line: Open V-205 \\
\hline 8 & 9 & $\begin{array}{l}\text { Record STSC Weight and level (WIT-740-401 and LIT-740-401). } \\
\text { Calculate and verify final buoyant weight of sludge transferred (DWI- } \\
740-401 \text { ). Begin preparation of shipping manifest. (Safety Significant } \\
\text { Measurement) }\end{array}$ & $\mathrm{N}$ & $\mathrm{Y}$ & $\begin{array}{l}\text { Added Instrument } \\
\text { designation }\end{array}$ & 9 & $\begin{array}{l}\text { Record STSC Weight and level. Calculate and verify final } \\
\text { buoyant weight of sludge transferred (DWI-740-401). Begin } \\
\text { preparation of shipping manifest }\end{array}$ \\
\hline 8 & 10 & $\begin{array}{l}\text { Unbolt the Sludge Inlet Connection from Nozzle A. Raise flange 2" to } \\
\text { open the connector internal vent port. Verify IL-118 turns OFF. }\end{array}$ & $\mathrm{N}$ & $\mathrm{N}$ & & 10 & $\begin{array}{l}\text { Unbolt the Sludge Inlet Connection. Raise flange } 2 " \text { to open the } \\
\text { connector internal vent port. Verify IL-118 turns OFF. }\end{array}$ \\
\hline 8 & 11 & $\begin{array}{l}\text { Verify positive airflow through TLSB high point vent (IL-125 turns } \\
\text { ON) }\end{array}$ & $\mathrm{N}$ & $\mathrm{N}$ & & 11 & $\begin{array}{l}\text { Verify positive airflow through TLSB high point vent (IL-125 } \\
\text { turns ON) }\end{array}$ \\
\hline 8 & $12 \mid \mathrm{r}$ & $\begin{array}{l}\text { Unbolt the Decant line Connection from Nozzle B. Raise flange } 2 " \text { to } \\
\text { open the connector internal vent port. Verify IL-218 turns OFF. }\end{array}$ & $\mathrm{N}$ & $\mathrm{N}$ & & 12 & $\begin{array}{l}\text { Unbolt the Decant/backwash line Connection. Raise flange 2" } \\
\text { to open the connector internal vent port. Verify IL-218 turns } \\
\text { OFF }\end{array}$ \\
\hline 8 & 13 & $\begin{array}{l}\text { Verify positive airflow through Decant box high point vent (IL-223 } \\
\text { turns ON) }\end{array}$ & $\mathrm{N}$ & $\mathrm{N}$ & & 13 & $\begin{array}{l}\text { Verify positive airflow through Decant box high point vent (IL- } \\
223 \text { turns ON) }\end{array}$ \\
\hline
\end{tabular}


PRC-STP-00473 REV 0

MODE 8 - DISCONNECT \& PURGE STSC

\begin{tabular}{|c|c|c|c|c|c|c|c|}
\hline Mode & Step & $\begin{array}{l}\text { Current Operations Sequence Description } \\
\text { (from PRC-STP-00358 Rev 1) }\end{array}$ & $\begin{array}{c}\text { Step } \\
\text { Change }\end{array}$ & $\begin{array}{l}\text { Content } \\
\text { Change }\end{array}$ & $\begin{array}{c}\text { Discussion } \\
\text { (basis for change) }\end{array}$ & & $\begin{array}{c}\text { Previous Step \& Description } \\
\text { (PRC-STP-00382) } \\
\end{array}$ \\
\hline 8 & 14 & $\begin{array}{l}\text { Note: At this point, air is flowing from the room into the decant box } \\
\text { and the TLSB through the HIH annulus, and from the air breaks in the } \\
\text { decant box and TLSB through the inner hose of HIH hoses H-107 and } \\
\text { H-203 into the STSC. Leave nozzles in this configuration until the } \\
\text { inner hoses are drip-free. }\end{array}$ & $\mathrm{N}$ & $\mathrm{Y}$ & Changed dry to drip-free. & 14 & $\begin{array}{l}\text { Note: At this point, air is flowing from the room into the decant } \\
\text { box and the TLSB through the HIH annulus, and from the air } \\
\text { breaks in the decant box and TLSB through the inner hose of } \\
\text { HIH hoses H-107 and H-203 into the STSC. Leave nozzles in } \\
\text { this configuration until the inner hoses are dry. }\end{array}$ \\
\hline 8 & 15 & $\begin{array}{l}\text { Disconnect the Sludge line connector from Nozzle A. Install 3-bolt } \\
\text { blind flange on Nozzle A. }\end{array}$ & $\mathrm{N}$ & $\mathrm{N}$ & & 15 & $\begin{array}{l}\text { Disconnect the Sludge line connector from Nozzle A. Install 3- } \\
\text { bolt blind flange on Nozzle A }\end{array}$ \\
\hline 8 & 16 & $\begin{array}{l}\text { Disconnect the Decant line connector from Nozzle B. Install 4-bolt } \\
\text { blind flange on Nozzle B. }\end{array}$ & $\mathrm{N}$ & $\mathrm{N}$ & & 16 & $\begin{array}{l}\text { Disconnect the Decant/backwash line connector from Nozzle B. } \\
\text { Install 4-bolt blind flange on Nozzle B }\end{array}$ \\
\hline 8 & 17 & $\begin{array}{l}\text { Note: At this point, air is flowing from the room up through both the } \\
\text { inner hose and the annulus of } \mathrm{H}-107 \text { and } \mathrm{H}-203 \text { into the TLSB and the } \\
\text { decant box. }\end{array}$ & $\mathrm{N}$ & $\mathrm{N}$ & & 17 & $\begin{array}{l}\text { Note: At this point, air is flowing from the room up through } \\
\text { both the inner hose and the annulus of } \mathrm{H}-107 \text { and } \mathrm{H}-203 \text { into } \\
\text { the TLSB and the decant box }\end{array}$ \\
\hline 8 & 18 & $\begin{array}{l}\text { Install the end caps and contamination controls on the sludge and } \\
\text { decant line connectors. IL-125 and IL-223 turn OFF. Place the } \\
\text { connectors in the storage position. }\end{array}$ & $\mathrm{N}$ & $\mathrm{N}$ & & 18 & $\begin{array}{l}\text { Install the end caps and contamination controls on the sludge } \\
\text { and decant/backwash line connectors. Place the connectors in } \\
\text { the storage position }\end{array}$ \\
\hline 8 & 19 & Secure from Sludge line draining/venting: Close V-109 & $\mathrm{N}$ & $\mathrm{N}$ & & 19 & Secure from Sludge line draining/venting: Close V-109 \\
\hline 8 & 20 & Secure from Decant line draining/venting: Close V-205 & $\mathrm{N}$ & $\mathrm{N}$ & & 20 & $\begin{array}{l}\text { Secure from Decant/backwash line draining/venting: Close V- } \\
205\end{array}$ \\
\hline 8 & 21 & $\begin{array}{l}\text { Disconnect the } 4 \text { inch STSC Vent connection and install the Camlok } \\
\text { cap on Nozzle F1 }\end{array}$ & $\mathrm{N}$ & $\mathrm{N}$ & & 21 & $\begin{array}{l}\text { Disconnect the } 4 \text { inch STSC Vent connection and install the } \\
\text { Camlok cap on Nozzle F1 }\end{array}$ \\
\hline 8 & 22 & Initiate STSC Inert Gas Purge: & $\mathbf{N}$ & $\mathrm{N}$ & & 22 & Initiate STSC Inert Gas Purge: \\
\hline 8 & 23 & Align inert gas 3-way valve V-609 to 'STSC' position & $\mathrm{N}$ & N & & 23 & Align inert gas 3-way valve V-609 to 'STSC' position \\
\hline 8 & 24 & Open V-608. Inert gas flows into the STSC. & $\mathrm{N}$ & $\mathrm{Y}$ & $\begin{array}{l}\text { V-610 deleted by design } \\
\text { progression }\end{array}$ & 24 & Open V-610 and V-608. Inert gas flows into the STSC \\
\hline 8 & 25 & Verify/adjust inert gas flow rate with rotameter FI-760-601 & $\mathrm{N}$ & $\mathrm{N}$ & & 25 & Verify/adjust inert gas flow rate with rotameter FI-760-601 \\
\hline 8 & 26 & Close V-732. Air purge flow stops. Verify IL-651 is ON. & $\mathrm{N}$ & $\mathrm{N}$ & & 26 & Close V-732. Air purge flow stops. Verify IL-651 is OFF. \\
\hline
\end{tabular}


PRC-STP-00473 REV 0

MODE 8 - DISCONNECT \& PURGE STSC

\begin{tabular}{|c|c|c|c|c|c|c|c|}
\hline Mode & Step & $\begin{array}{l}\text { Current Operations Sequence Description } \\
\text { (from PRC-STP-00358 Rev 1) }\end{array}$ & $\begin{array}{c}\text { Step } \\
\text { Change }\end{array}$ & $\begin{array}{l}\text { Content } \\
\text { Change }\end{array}$ & $\begin{array}{c}\text { Discussion } \\
\text { (basis for change) }\end{array}$ & & $\begin{array}{l}\text { Previous Step \& Description } \\
\text { (PRC-STP-00382) }\end{array}$ \\
\hline 8 & 27 & $\begin{array}{l}\text { Prior to securing from STSC Inerting, insure shipping window } \\
\text { availability. }\end{array}$ & N & $\mathrm{N}$ & & 27 & $\begin{array}{l}\text { Prior to securing from STSC Inerting, insure shipping window } \\
\text { availability. }\end{array}$ \\
\hline 8 & 28 & $\begin{array}{l}\text { Secure from STSC Inert Gas Purge upon } \mathrm{O}_{2} \text { Sensor AI-760-601 reading } \\
\text { less than } 1 \text { vol\%: Close V-608. Start the Cask Purge Clock. }\end{array}$ & $\mathrm{N}$ & $\mathrm{N}$ & & 28 & $\begin{array}{l}\text { Secure from STSC Inert Gas Purge upon O2 Sensor AI-760- } \\
601 \text { reading less than } 1 \text { vol\%: Close V-608 and V-610. Start the } \\
\text { Cask Purge Clock }\end{array}$ \\
\hline 8 & 29 & $\begin{array}{l}\text { Disconnect the Purge Outlet and install shipping Filter onto the } 2 \text { inch } \\
\text { Quick Connect Nozzle F2 }\end{array}$ & $\mathrm{N}$ & $\mathrm{N}$ & & 29 & $\begin{array}{l}\text { Disconnect the Purge Outlet and install shipping Filter onto the } \\
2 \text { inch Quick Connect Nozzle F2 }\end{array}$ \\
\hline 8 & 30 & $\begin{array}{l}\text { Disconnect the STSC Purge Gas inlet hose and install the shipping } \\
\text { filter onto the } 1 \text { inch Quick Connect Nozzle S2 }\end{array}$ & $\mathrm{N}$ & $\mathrm{Y}$ & Change 2 inch to 1 inch & 30 & $\begin{array}{l}\text { Disconnect the STSC Purge Gas inlet hose and install the } \\
\text { shipping filter onto the } 2 \text { inch Quick Connect Nozzle S2 }\end{array}$ \\
\hline 8 & 31 & $\begin{array}{l}\text { Disconnect I\&C connector to LDE-710-103 (Leak Detector) and LE- } \\
740-401 \text { (Level Detector) Safety Significant instruments }\end{array}$ & $\mathrm{N}$ & $\mathrm{N}$ & & 31 & $\begin{array}{l}\text { Disconnect I\&C connector to LDE-710-103 (Leak Detector) } \\
\text { and LE-740-401 (Level Detector) Safety Significant } \\
\text { instruments }\end{array}$ \\
\hline 8 & 32 & $\begin{array}{l}\text { Disconnect I\&C connector to LDE-720-204 (Leak Detector) and LSH- } \\
740-402 \text { (High Level Switch) General Service instruments }\end{array}$ & $\mathrm{N}$ & $\mathrm{N}$ & & 32 & $\begin{array}{l}\text { Disconnect I\&C connector to LDE-720-204 (Leak Detector) } \\
\text { and LSH-740-402 (High Level Switch) General Service } \\
\text { instruments }\end{array}$ \\
\hline 8 & 33 & $\begin{array}{l}\text { Concurrent with Purging operations, Inspect the STS Lid o-ring and } \\
\text { replace Helicoflex seal. }\end{array}$ & $\mathrm{N}$ & $\mathrm{N}$ & & 33 & $\begin{array}{l}\text { Concurrent with Purging operations, Inspect the STS Lid o-ring } \\
\text { and replace Helicoflex seal }\end{array}$ \\
\hline 8 & 34 & Install the STS Lid and torque Lid Bolts ( 24 bolts). & $\mathrm{N}$ & $\mathrm{N}$ & & 34 & Install the STS Lid and torque Lid Bolts \\
\hline 8 & 35 & Remove Lid Lift Fixture from STS cask lid & $\mathrm{N}$ & $\mathrm{N}$ & & 35 & Remove Lid Lift Fixture from STS cask lid \\
\hline 8 & 36 & Perform leak test on STS Lid: & $\mathrm{N}$ & $\mathrm{N}$ & & 36 & Perform leak test on STS Lid: \\
\hline 8 & 37 & Remove leak test plug & $\mathrm{N}$ & $\mathrm{N}$ & & 37 & Remove leak test plug \\
\hline 8 & 38 & Install leak test tool in test port & $\mathrm{N}$ & $\mathrm{N}$ & & 38 & Install leak test tool in test port \\
\hline 8 & 39 & Pressurize the lid seal annulus and monitor for specified time & $\mathrm{N}$ & $\mathrm{N}$ & & 39 & Pressurize the lid seal annulus and monitor for specified time \\
\hline
\end{tabular}


PRC-STP-00473 REV 0

MODE 9 - PURGE \& SHIP STS CASK

\begin{tabular}{|c|c|c|c|c|c|c|c|}
\hline Mode & Step & $\begin{array}{l}\text { Current Operations Sequence Description } \\
\text { (from PRC-STP-00358 Rev 1) } \\
\end{array}$ & $\begin{array}{c}\text { Step } \\
\text { Change }\end{array}$ & $\begin{array}{l}\text { Content } \\
\text { Change }\end{array}$ & $\begin{array}{c}\text { Discussion } \\
\text { (basis for change) } \\
\end{array}$ & & $\begin{array}{c}\text { Previous Step \& Description } \\
\text { (PRC-STP-00382) } \\
\end{array}$ \\
\hline 9 & 1 & Remove STS Cask vent port cap and install vent port tool & $\mathrm{Y}$ & No - was 4 & $\begin{array}{l}\text { Steps changed because } \\
\text { initial venting of STS Cask } \\
\text { was via the drain port rather } \\
\text { than vent port for Mode } 1 \text {. } \\
\text { Steps } 1 \text { thru } 3 \text { not needed. }\end{array}$ & 4 & Remove STS Cask vent port cap and install vent port tool \\
\hline 9 & 2 & Attach vent line $(\mathrm{H}-659)$ to vent port tool & $\mathrm{Y}$ & No - was 5 & & 5 & Attach vent line $(H-659)$ to vent port tool \\
\hline 9 & 3 & Open STS drain port and STS vent port & $\mathrm{Y}$ & No - was 6 & & \multirow[b]{2}{*}{6} & \multirow{2}{*}{$\begin{array}{l}\text { Open STS drain port and STS vent port \& Align inert gas 3- } \\
\text { way valve V-609 to 'STS Cask' position }\end{array}$} \\
\hline 9 & 4 & Align inert gas 3-way valve, V-609, to 'STS' position & Y & $\begin{array}{c}\text { No - was } \\
\text { included in } 6\end{array}$ & & & \\
\hline 9 & 5 & $\begin{array}{l}\text { Open V-608 to Purge STS cask with inert gas. Stop the Cask Purge } \\
\text { Clock. }\end{array}$ & Y & No - was 7 & & 7 & $\begin{array}{l}\text { Open V- } 608 \text { to Purge STS cask with inert gas. Stop the Cask } \\
\text { Purge Clock }\end{array}$ \\
\hline 9 & 6 & $\begin{array}{l}\text { Secure from STS Cask Inert Gas Purge upon } \mathrm{O}_{2} \text { Sensor AI- }-760-601 \\
\text { reading less than } 1 \text { vol\%: Close V-608. Start the Shipping Window } \\
\text { Clock }\end{array}$ & $\mathrm{Y}$ & No - was 8 & & 8 & $\begin{array}{l}\text { Secure from STS Cask Inert Gas Purge upon O2 Sensor AI- } \\
760-601 \text { reading less than } 1 \text { vol\%: Close V-608. Start the } \\
\text { Shipping Window Clock }\end{array}$ \\
\hline 9 & 7 & Close STS vent port & $\mathrm{Y}$ & No - was 9 & & 9 & Close STS vent port \\
\hline 9 & 8 & $\begin{array}{l}\text { Open needle valve V-607 to pressurize the STS with Inert Gas to } \\
\text { approximately } 3 \text { psi (PI-760-606). Close V-607. }\end{array}$ & $\mathrm{Y}$ & $Y$ - was 10 & $\begin{array}{l}\text { Added Instrument } \\
\text { designation }\end{array}$ & 10 & $\begin{array}{l}\text { Open needle valve V-607 to pressurize the STS with Inert Gas } \\
\text { to approximately } 3 \text { psi. Close V-607 }\end{array}$ \\
\hline 9 & 9 & Disconnect vent line from vent port tool & $\mathrm{Y}$ & No - was 11 & & 11 & Disconnect vent line from vent port tool \\
\hline 9 & 10 & Close STS drain port & $\mathrm{Y}$ & No - was 12 & & 12 & Close STS drain port \\
\hline 9 & 11 & $\begin{array}{l}\text { Relieve inert gas line pressure and disconnect inert gas supply line } \\
\text { from drain port tool }\end{array}$ & $\mathrm{Y}$ & No - was 13 & & 13 & $\begin{array}{l}\text { Relieve inert gas line pressure and disconnect inert gas supply } \\
\text { line from drain port tool }\end{array}$ \\
\hline 9 & 12 & Remove vent port tool and install vent port cap & $\mathrm{Y}$ & No - was 14 & & 14 & Remove vent port tool and install vent port cap \\
\hline 9 & 13 & Install/zip up Inner IP-2 STS lid bag & $\mathrm{Y}$ & No- was 15 & & 15 & Install/zip up Inner IP-2 STS lid bag \\
\hline
\end{tabular}




\section{PRC-STP-00473 REV 0}

MODE 9 - PURGE \& SHIP STS CASK

\begin{tabular}{|c|c|c|c|c|c|c|c|}
\hline Mode & Step & $\begin{array}{l}\text { Current Operations Sequence Description } \\
\text { (from PRC-STP-00358 Rev 1) }\end{array}$ & $\begin{array}{c}\text { Step } \\
\text { Change }\end{array}$ & $\begin{array}{l}\text { Content } \\
\text { Change }\end{array}$ & $\begin{array}{c}\text { Discussion } \\
\text { (basis for change) }\end{array}$ & & $\begin{array}{l}\text { Previous Step \& Description } \\
\text { (PRC-STP-00382) } \\
\end{array}$ \\
\hline 9 & 14 & Install/zip up Outer IP-2 STS lid bag & $\mathrm{Y}$ & No - was 16 & & 16 & Install/zip up Outer IP-2 STS lid bag \\
\hline 9 & 15 & Remove drain port tool and install drain port cap & $\mathrm{Y}$ & No - was 17 & & 17 & Remove drain port tool and install drain port cap \\
\hline 9 & 16 & Close/install IP-2 closure on STS drain port & $\mathrm{Y}$ & No - was 18 & & 18 & Close/install IP-2 closure on STS drain port \\
\hline 9 & 17 & Weigh and record final STS loaded weight (WIT-740-401). & $\mathrm{Y}$ & $\mathrm{Y}$ - was 19 & $\begin{array}{l}\text { Added Instrument } \\
\text { designation }\end{array}$ & 19 & Weigh and record final STS loaded weight \\
\hline 9 & 18 & Complete preparation of shipping manifest & $\mathrm{Y}$ & No - was 20 & & 20 & Complete preparation of shipping manifest \\
\hline 9 & 19 & Open loading bay door & $\mathrm{Y}$ & No - was 21 & & 21 & Open loading bay door \\
\hline 9 & 20 & Position tractor and connect to STS trailer & $\mathrm{Y}$ & No - was 22 & & 22 & Position tractor and connect to STS trailer \\
\hline 9 & 21 & Receive and verify shipping manifest & $\mathrm{Y}$ & No - was 23 & & 23 & Receive and verify shipping manifest \\
\hline 9 & 22 & Remove loaded STS trailer from annex loading bay & $\mathrm{Y}$ & No - was 24 & & 24 & Remove loaded STS trailer from annex loading bay \\
\hline 9 & 23 & Close loading bay door & $\mathrm{Y}$ & No - was 25 & & 25 & Close loading bay door \\
\hline 9 & 24 & Exit Annex loading bay & $\mathrm{Y}$ & No - was 26 & & 26 & Exit Annex loading bay \\
\hline \multicolumn{8}{|c|}{ 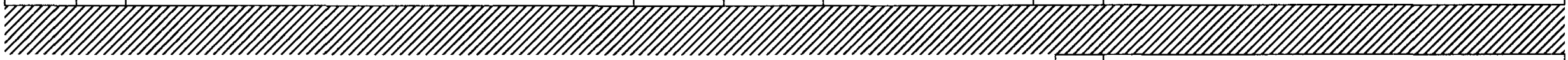 } \\
\hline \multicolumn{8}{|c|}{ 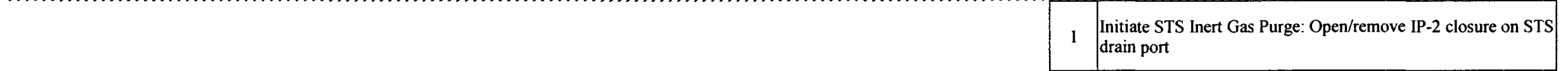 } \\
\hline & & & & & & 2 & Remove STS Cask drain port cap and install drain port tool. \\
\hline & & & & & & 3 & Attach inert gas line to drain port tool \\
\hline
\end{tabular}


PRC-STP-00473 REV 0

APPENDIX B

“WHAT/IF" HAZARD ANALYSIS MEETING PARTICIPANTS

B- $\mathrm{i}$ 


\begin{tabular}{|l|l|}
\hline \multicolumn{2}{|c|}{$\begin{array}{c}\text { ECRTS What/If Hazard Analysis } \\
\text { NPH, External Events, and Fire } \\
\text { September 10, 2010 }\end{array}$} \\
\hline \multicolumn{2}{|c|}{ Hazards Analysis Team } \\
\hline Robert Meichle & \multicolumn{1}{c|}{ Organization } \\
\hline Gary Franz & Facilitator, STP NS \\
\hline Dewey Burbank & Facilitator/Scribe, STP NS \\
\hline Ralph Crowe & STP/ECRTS Eng \\
\hline Gail Chaffee & STP NS \\
\hline Lindsay Nelsen & 100K NS \\
\hline Jeff Broussard & STP Rad Control \\
\hline James Mathews & 100K Ops \\
\hline Darren Schwartz & STP Ops \\
\hline Brett Kujath/Susan Omberg Carro & $100 \mathrm{~K}$ OS/IH \\
\hline David Watson & STP Fire Protection \\
\hline Raymond Puigh & STP Environmental \\
\hline William Edwards & Crit Safety \\
\hline \hline Additional Attendees & Transportation \\
\hline Dennis Hamilton & \\
\hline Craig Carro & STP/ECRTS Manager \\
\hline Richard Raymond & STP NS \\
\hline Al Bridges & STP Chief Engineer \\
\hline John Dearing & CHPRC Transportation Safety \\
\hline Jim Criddle & STP Sampling \& Characterization Eng \\
\hline Hall, Mark A & STP/ECRTS Eng \\
\hline Geoff Barnes & STP/ECRTS Eng \\
\hline Dennis Markman & STP/ECRTS Eng \\
\hline & STP/ECRTS Eng \\
\hline \multicolumn{2}{|c|}{ Nand } \\
\hline
\end{tabular}


PRC-STP-00473 REV 0

\begin{tabular}{|l|l|}
\hline \multicolumn{2}{|c|}{$\begin{array}{c}\text { ECRTS What/If Hazard Gap Analysis } \\
\text { October 19, 2010 } \\
\text { Meeting Participants }\end{array}$} \\
\hline \multicolumn{2}{|c|}{ Hazards Analysis Team } \\
\hline Robert Meichle & \multicolumn{1}{c|}{ Organization } \\
\hline Gary Franz & Facilitator, STP NS \\
\hline Dewey Burbank & Facilitator/Scribe, STP NS \\
\hline Craig Carro & STP/ECRTS Eng \\
\hline Lindsay Nelsen & STP NS \\
\hline James Mathews & STP Rad Control \\
\hline Darren Schwartz & STP Ops \\
\hline Brett Kujath/Susan Omberg Carro & 100K OS/IH \\
\hline \hline \multicolumn{2}{|c|}{ Additional Attendees } \\
\hline Dennis Hamilton & STP Fire Protection \\
\hline
\end{tabular}


PRC-STP-00473 REV 0

\begin{tabular}{|l|l|}
\hline \multicolumn{2}{|c|}{$\begin{array}{c}\text { ECRTS What/If Hazard Gap Analysis } \\
\text { Reconvene October 22, 2010 } \\
\text { Meeting Participants }\end{array}$} \\
\hline \multicolumn{2}{|c|}{ Hazards Analysis Team } \\
\hline Robert Meichle & \multicolumn{1}{c|}{ Organization } \\
\hline Gary Franz & Facilitator, STP NS \\
\hline Dewey Burbank & Facilitator/Scribe, STP NS \\
\hline Ralph Crowe & STP/ECRTS Eng \\
\hline Lindsay Nelsen & STP NS \\
\hline Gary Hastings & STP Rad Control \\
\hline James Mathews & $100 \mathrm{~K}$ Rad Control \\
\hline Darren Schwartz & STP Ops \\
\hline Brett Kujath/Susan Omberg Carro & $100 \mathrm{~K}$ OS/IH \\
\hline \hline Additional Attendees & STP Fire Protection \\
\hline Dennis Hamilton & \\
\hline Craig Carro & STP/ECRTS Manager \\
\hline Dan McHugh & STP NS \\
\hline Dennis Clapp (part time) & STP NS \\
\hline Greg Morgan (part time) & DOE-RL \\
\hline
\end{tabular}


PRC-STP-00473 REV 0

APPENDIX C

"WHAT/IF" HAZARD ANALYSIS RESULTS

NPH AND EXTERNAL EVENTS 


\begin{tabular}{|c|c|c|c|c|c|c|c|}
\hline \multicolumn{8}{|c|}{ TABLE C-1 "WHAT/IF" HAZARD ANALYSIS STUDY RESULTS - NPH AND EXTERNAL EVENTS } \\
\hline Identifier & "What/If" & Causes & Hazardous Condition & Consequences & $\begin{array}{l}\text { Candidate Controls } \\
\text { Engineered Features }\end{array}$ & Admin Controls & Remarks/Actions \\
\hline \multicolumn{8}{|c|}{ SEISMIC } \\
\hline S-1 & $\begin{array}{l}\text { Modified Annex structure } \\
\text { or crane collapses due to } \\
\text { basin structure failure } \\
\text { prior to sludge retrieval } \\
\text { and loading }\end{array}$ & NPH Seismic & Industrial hazard & $\begin{array}{l}\text { Personnel hazard, programmatic impact from } \\
\text { structural collapse or equipment damage }\end{array}$ & $\begin{array}{l}\text { Structure and equipment designed to International } \\
\text { Building Code or better. }\end{array}$ & None & \\
\hline $\mathrm{s}-2$ & $\begin{array}{l}\text { Modified Annex structure } \\
\text { or crane collapses due to } \\
\text { basin structure failure } \\
\text { during sludge retrieval } \\
\text { and loading or overfill } \\
\text { recovery }\end{array}$ & NPH Seismic & $\begin{array}{l}\text { Spray leak } \\
\text { Splash and splatter }\end{array}$ & $\begin{array}{l}\text { Impact on transfer line in annex leads to } \\
\text { Uncontrolled release, worker and public dose. }\end{array}$ & $\begin{array}{l}\text { Seismic separation between Annex Structure and } \\
\text { Basin structure to prevent interaction, even if basin } \\
\text { structure fails. } \\
\text { Seismic switch, } \\
\text { Dead man operating switch, } \\
\text { Pump operating timer. }\end{array}$ & None & $\begin{array}{l}\text { Annex structure damage includes potential } \\
\text { failure of any of the walls or internal walls } \\
\text { and upper levels or roof or valve boxes, } \\
\text { and where applicable, ventilation stack or } \\
\text { ducting/equipment. Secondary } \\
\text { confinement, hose barn, leak detection, } \\
\text { interlocks, ventilation confinement, fire } \\
\text { protection are assumed to fail to function } \\
\text { during unmitigated seismic event. }\end{array}$ \\
\hline S-3 & $\begin{array}{l}\text { KW Basin super-structure } \\
\text { failure during sludge } \\
\text { retrieval and loading or } \\
\text { overfill recovery }\end{array}$ & NPH Seismic & $\begin{array}{l}\text { Spray leak } \\
\text { Splash and splatter }\end{array}$ & $\begin{array}{l}\text { Impact on transfer line outside basin and } \\
\text { outside annex leads to Uncontrolled release of } \\
\text { sludge, worker and public dose. }\end{array}$ & $\begin{array}{l}\text { Hose barn design prevents impact, } \\
\text { Seismic switch, } \\
\text { Dead man operating switch, } \\
\text { Pump operating timer, }\end{array}$ & None & \\
\hline S-4 & $\begin{array}{l}\text { Modified Annex } \\
\text { Structure or crane } \\
\text { collapses during transfer } \\
\text { or overfill recovery }\end{array}$ & NPH Seismic & $\begin{array}{l}\text { Spray leak } \\
\text { Splash and splatter }\end{array}$ & $\begin{array}{l}\text { Impact on transfer line in annex leads to } \\
\text { Uncontrolled release of sludge, worker and } \\
\text { public dose. }\end{array}$ & $\begin{array}{l}\text { Seismic qualification of structure or hose pathway } \\
\text { so as to protect from structure, } \\
\text { Seismic switch, } \\
\text { Dead man operating switch, } \\
\text { Pump operating timer. }\end{array}$ & None & \\
\hline S-5 & $\begin{array}{l}\text { Hoses disconnected at } \\
\text { transfer box during } \\
\text { transfer or overfill } \\
\text { recovery }\end{array}$ & NPH Seismic & $\begin{array}{l}\text { Spray leak } \\
\text { Splash and splatter }\end{array}$ & $\begin{array}{l}\text { Transfer line failure leading to Uncontrolled } \\
\text { release of sludge, worker and public dose. }\end{array}$ & $\begin{array}{l}\text { Seismic qualification of structure and transfer box, } \\
\text { Seismic switch, } \\
\text { Dead man operating switch, } \\
\text { Pump operating timer. }\end{array}$ & None & \\
\hline $\begin{array}{ll}\text { S-6 } \\
\end{array}$ & $\begin{array}{l}\text { Modified Annex structure } \\
\text { collapses due to basin } \\
\text { structure failure during } \\
\text { decant }\end{array}$ & NPH Seismic & $\begin{array}{l}\text { Spray leak } \\
\text { Splash and splatter }\end{array}$ & $\begin{array}{l}\text { Impact on decant/backwash line in annex } \\
\text { leads to Uncontrolled release of sludge, } \\
\text { worker and public dose. }\end{array}$ & $\begin{array}{l}\text { Seismic qualification of structure and decant box, } \\
\text { Seismic switch, } \\
\text { Dead man operating switch, } \\
\text { pump operating timer. }\end{array}$ & None & \\
\hline S-7 & $\begin{array}{l}\text { Basin super-structure } \\
\text { collapses during decant }\end{array}$ & NPH Seismic & $\begin{array}{l}\text { Spray leak } \\
\text { Splash and splatter }\end{array}$ & $\begin{array}{l}\text { After decant filtration, impact on } \\
\text { decantbackwash line above basin water or } \\
\text { outside annex leads to Uncontrolled release of } \\
\text { sludge }\end{array}$ & $\begin{array}{l}\text { Hose barn design prevents impact, } \\
\text { Seismic switch, } \\
\text { Dead man operating switch, } \\
\text { Pump operating timer. }\end{array}$ & None & \\
\hline S-8 & $\begin{array}{l}\text { Hoses disconnected at } \\
\text { decant box or sand filter } \\
\text { or STSC during decant or } \\
\text { circulation to inject floc }\end{array}$ & NPH Seismic & $\begin{array}{l}\text { Spray leak } \\
\text { Splash and splatter }\end{array}$ & $\begin{array}{l}\text { Decant/backwash line failure leading to } \\
\text { Uncontrolled release of sludge, worker and } \\
\text { public dose. }\end{array}$ & $\begin{array}{l}\text { Seismic qualification of structure and decant box } \\
\text { and STSC and sand filter and hoses, } \\
\text { Seismic switch, } \\
\text { Dead man operating switch, } \\
\text { Pump operating timer. }\end{array}$ & None & \\
\hline
\end{tabular}




\begin{tabular}{|c|c|c|c|c|c|c|c|}
\hline \multirow[t]{2}{*}{ Identifier } & \multirow[t]{2}{*}{ "What/If" } & \multirow[t]{2}{*}{ Causes } & \multirow[t]{2}{*}{ Hazardous Condition } & \multirow[t]{2}{*}{ Consequences } & \multicolumn{2}{|l|}{ Candidate Controls } & \multirow[t]{2}{*}{ Remarks/Actions } \\
\hline & & & & & Engineered Features & Admin Controls & \\
\hline S-9 & $\begin{array}{l}\text { Modified Annex structure } \\
\text { collapses due to } \mathrm{KW} \\
\text { basin super-structure } \\
\text { failure during backwash }\end{array}$ & NPH Seismic & $\begin{array}{l}\text { Spray leak } \\
\text { Splash and splatter }\end{array}$ & $\begin{array}{l}\text { Impact on decant/backwash line in annex } \\
\text { leads to Uncontrolled release of sludge, } \\
\text { worker and public dose. }\end{array}$ & $\begin{array}{l}\text { Seismic separation between Annex Structure and } \\
\text { Basin structure to prevent interaction, even if basin } \\
\text { structure fails. } \\
\text { Seismic switch, } \\
\text { Dead man operating switch, } \\
\text { Pump operating timer. }\end{array}$ & None & \\
\hline S-10 & $\begin{array}{l}\text { Modified Annex structure } \\
\text { collapses during } \\
\text { backwash }\end{array}$ & NPH Seismic & $\begin{array}{l}\text { Spray leak } \\
\text { Splash and splatter }\end{array}$ & $\begin{array}{l}\text { Impact on decant/backwash line in annex } \\
\text { leads to Uncontrolled release of sludge, } \\
\text { worker and public dose. }\end{array}$ & $\begin{array}{l}\text { Seismic qualification of structure, } \\
\text { Seismic switch, } \\
\text { Dead man operating switch, } \\
\text { Pump operating timer. }\end{array}$ & None & \\
\hline S-11 & $\begin{array}{l}\text { Hoses disconnected at } \\
\text { sand filter or decant box } \\
\text { or STSC during backwash }\end{array}$ & NPH Seismic & $\begin{array}{l}\text { Spray leak } \\
\text { Splash and splatter }\end{array}$ & $\begin{array}{l}\text { Decant/backwash line failure leading to } \\
\text { Uncontrolled release of sludge, worker and } \\
\text { public dose. }\end{array}$ & $\begin{array}{l}\text { Seismic qualification of structure and decant box } \\
\text { and STSC and sand filter and hoses, } \\
\text { Seismic switch, } \\
\text { Dead man operating switch, } \\
\text { Pump operating timer. }\end{array}$ & None & \\
\hline S-12 & $\begin{array}{l}\text { Modified Annex } \\
\text { Structure collapses due to } \\
\text { KW basin super-structure } \\
\text { failure after sludge } \\
\text { retrieval and loading }\end{array}$ & NPH Seismic & Deflagration in STSC & $\begin{array}{l}\text { Impact to ventilation hoses, ducting, stack, or } \\
\text { ventilation equipment shuts down STSC } \\
\text { headspace active ventilation, resulting in } \\
\text { buildup of hydrogen leading to Uncontrolled } \\
\text { release of sludge, worker and public dose. }\end{array}$ & $\begin{array}{l}\text { Seismic separation between Annex Structure and } \\
\text { Basin structure to prevent interaction, even if basin } \\
\text { structure fails. }\end{array}$ & $\begin{array}{l}\text { Administrative } \\
\text { requirement to establish } \\
\text { purge following loss of } \\
\text { ventilation. }\end{array}$ & \\
\hline S-13 & $\begin{array}{l}\text { Modified Annex } \\
\text { Structure collapse or } \\
\text { crane drop after sludge } \\
\text { retrieval and loading }\end{array}$ & NPH Seismic & Deflagration in STSC & $\begin{array}{l}\text { Impact to ventilation hoses, ducting or } \\
\text { ventilation equipment shuts down STSC } \\
\text { headspace active ventilation, resulting in } \\
\text { buildup of hydrogen leading to Uncontrolled } \\
\text { release of sludge, worker and public dose. }\end{array}$ & $\begin{array}{l}\text { Seismic qualification of annex structure and } \\
\text { ventilation system }\end{array}$ & $\begin{array}{l}\text { Administrative } \\
\text { requirement to establish } \\
\text { purge following loss of } \\
\text { ventilation. }\end{array}$ & \\
\hline S-14 & $\begin{array}{l}\text { Modified Annex } \\
\text { Structure collapse or } \\
\text { crane drop or cask lid } \\
\text { drop during installation }\end{array}$ & NPH Seismic & Deflagration in STSC, STS & $\begin{array}{l}\text { Impact to inerting equipment or STSC nozzles } \\
\text { prevents purge and seal, leading to buildup of } \\
\text { hydrogen and uncontrolled release of sludge, } \\
\text { worker and public dose if STSC headspace } \\
\text { involved. }\end{array}$ & $\begin{array}{l}\text { Seismic qualification of annex structure, inerting } \\
\text { equipment and crane. }\end{array}$ & None & \\
\hline S-15 & $\begin{array}{l}\text { Modified Annex } \\
\text { Structure damage, trailer } \\
\text { damage or door damage } \\
\text { preventing load departure }\end{array}$ & NPH Seismic & cask overpressure & $\begin{array}{l}\text { STS load heats-up, hydrogen gas is generated } \\
\text { leading to uncontrolled release of sludge, } \\
\text { worker and public dose. }\end{array}$ & $\begin{array}{l}\text { Seismic qualification of structure, door and } \\
\text { STSC/STS/trailer. }\end{array}$ & $\begin{array}{l}\text { Administrative } \\
\text { requirement to establish } \\
\text { vent (and purge as } \\
\text { required) if shipping } \\
\text { delayed. }\end{array}$ & \\
\hline S-16 & $\begin{array}{l}\text { Damage to nitrogen bottle } \\
\text { rack leads to generation } \\
\text { of bottle missile }\end{array}$ & NPH Seismic & $\begin{array}{l}\text { Spray leak } \\
\text { Splash and splatter } \\
\text { Deflagration in STSC, STS }\end{array}$ & $\begin{array}{l}\text { Potential to impact to transfer lines, } \\
\text { ventilation equipment leading to event } \\
\text { comparable to S-4, 5, 8, 10,11, 13,14 and } \\
\text { uncontrolled release of sludge, worker and } \\
\text { public dose. }\end{array}$ & $\begin{array}{l}\text { Seismic qualification of structure, bottle rack, } \\
\text { STSC/STS/Trailer, inerting equipment. }\end{array}$ & $\begin{array}{l}\text { Administrative } \\
\text { requirement to establish } \\
\text { purge if ventilation lost, } \\
\text { Administrative } \\
\text { requirement to establish } \\
\text { vent (and purge as } \\
\text { required) if shipping } \\
\text { delayed. }\end{array}$ & \\
\hline
\end{tabular}




\begin{tabular}{|c|c|c|c|c|c|c|c|}
\hline \multicolumn{8}{|c|}{ TABLE C-1 “WHAT/IF” HAZARD ANALYSIS STUDY RESULTS - NPH AND EXTERNAL EVENTS } \\
\hline Identifier & "What/If" & Causes & Hazardous Condition & Consequences & $\begin{array}{c}\text { Candidate Controls } \\
\text { Engineered Features }\end{array}$ & Admin Controls & Remarks/Actions \\
\hline S-17 & $\begin{array}{l}\text { Damage to } \mathrm{T} \text { Plant or } \\
\text { travel route significantly } \\
\text { delays transfer }\end{array}$ & $\begin{array}{l}\text { Seismic } \\
\text { event }\end{array}$ & $\begin{array}{l}\text { STS load heats up, hydrogen } \\
\text { gas is generated leading to } \\
\text { cask overpressure }\end{array}$ & $\begin{array}{l}\text { Spread of contamination, worker and public } \\
\text { dose. }\end{array}$ & $\begin{array}{l}\text { Seismic qualification of inerting equipment, } \\
\text { STS/Trailer. }\end{array}$ & $\begin{array}{l}\text { Administrative } \\
\text { requirement to establish } \\
\text { vent (and purge as } \\
\text { required) if shipping } \\
\text { delayed. }\end{array}$ & \\
\hline S-18 & Damage to KW Stack & $\begin{array}{l}\text { Seismic } \\
\text { event }\end{array}$ & $\begin{array}{l}\text { Postulate impact to transfer } \\
\text { line or annex }\end{array}$ & N/A. & None & None & $\begin{array}{l}\text { Scale drawing demonstrates the } 175 \text { ' stack } \\
\text { does not reach new annex structure and } \\
\text { would not reach the transfer path from the } \\
\text { basin to the annex }\end{array}$ \\
\hline S-19 & $\begin{array}{l}\text { STSC overturns after } \\
\text { loading }\end{array}$ & $\begin{array}{l}\text { Seismic } \\
\text { event }\end{array}$ & $\begin{array}{l}\text { Spill of sludge into loading } \\
\text { bay }\end{array}$ & $\begin{array}{l}\text { Spread of contamination, worker and public } \\
\text { dose. }\end{array}$ & Seismic qualification of STSC/Trailer & None & \\
\hline S-20 & Fire initiated by DBE & $\begin{array}{l}\text { Seismic } \\
\text { event }\end{array}$ & $\begin{array}{l}\text { Fire events FF-1 through } \\
\text { FF-7 below are initiated by } \\
\text { and taken in combination } \\
\text { with seismic event failures }\end{array}$ & $\begin{array}{l}\text { Spread of contamination, worker and public } \\
\text { dose. }\end{array}$ & $\begin{array}{l}\text { Seismic qualification of structure and equipment } \\
\text { and fire suppression system, fire protection features } \\
\text { as indicated under FF-1 through FF-7. Seismic } \\
\text { switch, or dead man switch or timer limit spray or } \\
\text { splash and splatter in this event. }\end{array}$ & $\begin{array}{l}\text { Administrative limit on } \\
\text { combustibles }\end{array}$ & \\
\hline S-21 & $\begin{array}{l}\text { LOEP initiated by DBE } \\
\text { (Structural Damage to } \\
\text { electrical equipment) }\end{array}$ & $\begin{array}{l}\text { Seismic } \\
\text { event }\end{array}$ & $\begin{array}{l}\text { LOEP-1 through LOEP- } 5 \\
\text { events below are initiated by } \\
\text { and taken in combination } \\
\text { with seismic event failures }\end{array}$ & $\begin{array}{l}\text { Spread of contamination, worker and public } \\
\text { dose. (See LOEP events below for failure } \\
\text { configurations) }\end{array}$ & $\begin{array}{l}\text { Seismic design of required equipment under LOEP- } \\
1 \text { through LOEP- } 5 \text { to ensure availability. }\end{array}$ & None & 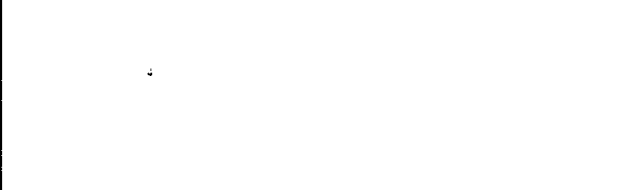 \\
\hline S-22 & $\begin{array}{l}\text { DBE drops equipment } \\
\text { into basin(Structural } \\
\text { failure-Equipment falls } \\
\text { into basin) }\end{array}$ & $\begin{array}{l}\text { Seismic } \\
\text { Event }\end{array}$ & $\begin{array}{l}\text { Impact to installed } \\
\text { equipment during transfer }\end{array}$ & $\begin{array}{l}\text { Operational upset. Leak sludge to basin water, } \\
\text { loss of visibility, delay in transfer to repair } \\
\text { equipment. }\end{array}$ & None & None & \\
\hline S-23 & DBE drops floc into basin & $\begin{array}{l}\text { Seismic } \\
\text { event }\end{array}$ & $\begin{array}{l}\text { Floc addition to stored } \\
\text { sludge and filters in IWTS }\end{array}$ & $\begin{array}{l}\text { Operational upset. May impact sludge } \\
\text { mobility or IWTS operability. }\end{array}$ & None & None & \\
\hline S-24 & $\begin{array}{l}\text { DBE leads to } \\
\text { LOP(Structural Damage } \\
\text { to instrument air } \\
\text { equipment) }\end{array}$ & $\begin{array}{l}\text { Seismic } \\
\text { Event }\end{array}$ & $\begin{array}{l}\text { Impact to instrument air } \\
\text { system components leads to } \\
\text { loss of pneumatics during } \\
\text { any system operation/ } \\
\text { configuration and taken in } \\
\text { combination with seismic } \\
\text { event failures }\end{array}$ & $\begin{array}{l}\text { Spread of contamination, worker and public } \\
\text { dose. (See LOP event below for failure } \\
\text { configurations) }\end{array}$ & Seismic qualification of structure and equipment. & None & $\begin{array}{l}\text { LOP events will be evaluated as part of } \\
\text { FMEA. Valves are currently configured to } \\
\text { prevent casualty. }\end{array}$ \\
\hline S-25 & $\begin{array}{l}\text { Seismic event results in } \\
\text { damage to annex structure } \\
\text { or equipment }\end{array}$ & $\begin{array}{l}\text { Seismic } \\
\text { Event }\end{array}$ & $\begin{array}{l}\text { Seismic event, perhaps less } \\
\text { than DBE, leads to } \\
\text { unanalyzed safety of } \\
\text { structure or equipment for } \\
\text { continued operation. }\end{array}$ & Operational upset, programmatic delay & None & None & \\
\hline
\end{tabular}




\begin{tabular}{|c|c|c|c|c|c|c|c|}
\hline \multicolumn{8}{|c|}{ TABLE C-1 “WHAT/IF" HAZARD ANALYSIS STUDY RESULTS - NPH AND EXTERNAL EVENTS } \\
\hline Identifier & "What/If" & Causes & Hazardous Condition & Consequences & $\begin{array}{c}\text { Candidate Controls } \\
\text { Engineered Features }\end{array}$ & Admin Controls & Remarks/Actions \\
\hline S-26 & $\begin{array}{l}\text { Loss of backup power } \\
\text { (Structural failure of } \\
\text { electrical equipment) }\end{array}$ & $\begin{array}{l}\text { Seismic } \\
\text { Event }\end{array}$ & $\begin{array}{l}\text { Common cause LOEP and } \\
\text { loss of backup power, } \\
\text { leading to STSC heat up, } \\
\text { hydrogen buildup } \\
\text { Deflagration in STSC, STS }\end{array}$ & $\begin{array}{l}\text { Spread of contamination, worker and public } \\
\text { dose. }\end{array}$ & $\begin{array}{l}\text { Covered in S-21 with qualification of backup power } \\
\text { for DBE. }\end{array}$ & None & \\
\hline S-27 & $\begin{array}{l}\text { Seismic event causes } \\
\text { Modified Annex structure } \\
\text { or equipment collapse on } \\
\text { to transfer, decant or } \\
\text { overfill recovery transfer } \\
\text { lines } \\
\end{array}$ & NPH Seismic & $\begin{array}{l}\text { Spray leak in an interfacing } \\
\text { line } \\
\text { Splash and splatter release } \\
\text { in an interfacing line. }\end{array}$ & $\begin{array}{l}\text { Transfer line blockage results in high pressure } \\
\text { and backward flow in lines interfacing with } \\
\text { the transfer line, causing an Uncontrolled } \\
\text { release of sludge, worker and public dose. }\end{array}$ & $\begin{array}{l}\text { Seismically qualified Modified Annex structure and } \\
\text { equipment } \\
\text { Block/Check valves prevent backflow in interfacing } \\
\text { systems, } \\
\text { Seismic switch, } \\
\text { Dead man switch. } \\
\end{array}$ & None & \\
\hline S-28 & $\begin{array}{l}\text { Seismic Event fails the } \\
\text { lighting system in the } \\
\text { annex }\end{array}$ & NPH Seismic & Industrial hazard & $\begin{array}{l}\text { Personnel within facility and on high } \\
\text { platforms are left without light causing } \\
\text { operational upset and personnel safety hazard }\end{array}$ & None & None & \\
\hline \multicolumn{8}{|l|}{ WIND } \\
\hline W-1 & $\begin{array}{l}\text { Modified Annex structure } \\
\text { or crane collapses due to } \\
\mathrm{KW} \text { basin super-structure } \\
\text { failure prior to sludge } \\
\text { retrieval and loading }\end{array}$ & High wind & $\begin{array}{l}\text { Industrial hazard, } \\
\text { facility impact from } \\
\text { structural collapse }\end{array}$ & $\begin{array}{l}\text { Personnel injury or fatality, programmatic } \\
\text { impact }\end{array}$ & Wind damage separation of structures & None & $\begin{array}{l}\text { Basin structure qualified to } 100 \mathrm{MPH} \text { and } \\
\text { for current PC-3 missile load - not to } 111 \\
\text { mph PC-3 wind for ECRTS major } \\
\text { modification. Thus, a potential for wind } \\
\text { load failure of the basin structure impact to } \\
\text { the new annex structure or the transfer line } \\
\text { exists. }\end{array}$ \\
\hline $\mathrm{W}-2$ & $\begin{array}{l}\text { Modified Annex structure } \\
\text { or crane collapses due to } \\
\mathrm{KW} \text { basin super-structure } \\
\text { failure during sludge } \\
\text { retrieval and loading, } \\
\text { decant or overfill } \\
\text { recovery }\end{array}$ & High wind & $\begin{array}{l}\text { Spray leak } \\
\text { Splash and splatter }\end{array}$ & $\begin{array}{l}\text { Impact on transfer line in annex leads to } \\
\text { Uncontrolled release of sludge, worker and } \\
\text { public dose. }\end{array}$ & $\begin{array}{l}\text { Structural separation and/or } \\
\text { structural qualification of annex to withstand } \\
\text { impact. }\end{array}$ & None & $\begin{array}{l}\text { Annex structure damage includes potential } \\
\text { failure of any of the walls and upper } \\
\text { levels or roof or support for valve boxes, } \\
\text { and where applicable, ventilation stack or } \\
\text { ducting/equipment. Secondary } \\
\text { confinement, ventilation confinement, fire } \\
\text { protection, instrumentation, interlocks } \\
\text { could variously fail to function due to } \\
\text { structure damage from high wind loads or } \\
\text { from wind borm missile. }\end{array}$ \\
\hline W-3 & $\begin{array}{l}\text { KW Basin super-structure } \\
\text { failure during sludge } \\
\text { retrieval and loading, } \\
\text { decant or overfill } \\
\text { recovery }\end{array}$ & High wind & \begin{tabular}{|l|} 
Spray leak \\
Splash and splatter
\end{tabular} & $\begin{array}{l}\text { Impact on transfer line above basin water and } \\
\text { outside annex leads to Uncontrolled release of } \\
\text { sludge, worker and public dose. }\end{array}$ & Hose barn PC-3 design prevents impact. & $\begin{array}{l}\text { Administrative control } \\
\text { to shut down transfers } \\
\text { during extreme weather } \\
\text { conditions if } \\
\text { structure/barn are not } \\
\text { qualified. } \\
\end{array}$ & $\begin{array}{l}\text { This kind of wind would certainly generate } \\
\text { an adverse weather advisory, an operations } \\
\text { shutdown, and likely a take cover. }\end{array}$ \\
\hline W-4 & $\begin{array}{l}\text { Missile impact to sludge } \\
\text { transfer lines external to } \\
\text { the Modified Annex } \\
\text { during loading, decant or } \\
\text { overfill recovery }\end{array}$ & $\begin{array}{l}\begin{array}{l}\text { High wind, } \\
\text { missile }\end{array} \\
\end{array}$ & $\begin{array}{l}\text { Spray leak } \\
\text { Splash and splatter }\end{array}$ & $\begin{array}{l}\text { Impact on transfer line above basin water and } \\
\text { outside annex leads to Uncontrolled release of } \\
\text { sludge, worker and public dose. }\end{array}$ & Hose barn PC-3 design prevents impact. & $\begin{array}{l}\text { Administrative control } \\
\text { to shut down transfers } \\
\text { during extreme weather } \\
\text { conditions if } \\
\text { structure/barn are not } \\
\text { qualified. } \\
\end{array}$ & $\begin{array}{l}\text { This kind of wind would certainly generate } \\
\text { an adverse weather advisory, an operations } \\
\text { shutdown, and likely a take cover. }\end{array}$ \\
\hline
\end{tabular}




\begin{tabular}{|c|c|c|c|c|c|c|c|}
\hline \multicolumn{8}{|c|}{ TABLE C-1 "WHAT/IF" HAZARD ANALYSIS STUDY RESULTS - NPH AND EXTERNAL EVENTS } \\
\hline Identifier & "What/If" & Causes & Hazardous Condition & Consequences & $\begin{array}{l}\text { Candidate Controls } \\
\text { Engineered Features }\end{array}$ & Admin Controls & Remarks/Actions \\
\hline W-5 & $\begin{array}{l}\text { Modified Annex } \\
\text { Structure collapses during } \\
\text { transfer, decant or overfill } \\
\text { recovery }\end{array}$ & High wind & $\begin{array}{l}\text { Spray leak } \\
\text { Splash and splatter }\end{array}$ & $\begin{array}{l}\text { Impact on transfer line in annex leads to } \\
\text { Uncontrolled release of sludge, worker and } \\
\text { public dose. }\end{array}$ & Structure qualified to withstand PC-3 wind & $\begin{array}{l}\text { Administrative control } \\
\text { to shut down transfers } \\
\text { during extreme weather } \\
\text { conditions. }\end{array}$ & $\begin{array}{l}\text { This kind of wind would certainly generate } \\
\text { an adverse weather advisory, an operations } \\
\text { shutdown, and likely a take cover. }\end{array}$ \\
\hline W-6 & $\begin{array}{l}\text { Hoses impacted or } \\
\text { disconnected at transfer } \\
\text { box,sand filter, decant } \\
\text { box or STSC during } \\
\text { transfer, decant, } \\
\text { backwash, or overfill } \\
\text { recovery } \\
\end{array}$ & $\begin{array}{l}\begin{array}{l}\text { High wind } \\
\text { missile }\end{array} \\
\end{array}$ & $\begin{array}{l}\text { Spray leak } \\
\text { Splash and splatter }\end{array}$ & $\begin{array}{l}\text { Transfer, decant/backwash line failure leading } \\
\text { to Uncontrolled release of sludge, worker and } \\
\text { public dose. }\end{array}$ & Structure qualified to withstand PC-3 missile & $\begin{array}{l}\text { Administrative control } \\
\text { to shut down transfers } \\
\text { during extreme weather } \\
\text { conditions. }\end{array}$ & $\begin{array}{l}\text { This kind of wind would certainly generate } \\
\text { an adverse weather advisory, an operations } \\
\text { shutdown, and likely a take cover. }\end{array}$ \\
\hline W-7 & $\begin{array}{l}\text { Modified Annex } \\
\text { Structure failure onto } \\
\text { loaded STSC }\end{array}$ & High wind & $\begin{array}{l}\text { Spill } \\
\text { Splash and splatter }\end{array}$ & $\begin{array}{l}\text { Overturn trailer, spill contents leading to } \\
\text { Uncontrolled release of sludge, worker and } \\
\text { public dose. }\end{array}$ & \begin{tabular}{|l} 
Structure qualified to withstand $\mathrm{PC}-3$ wind. \\
\end{tabular} & $\begin{array}{l}\text { Administrative control } \\
\text { to shut down transfers } \\
\text { during extreme weather } \\
\text { conditions. }\end{array}$ & $\begin{array}{l}\text { This kind of wind would certainly generate } \\
\text { an adverse weather advisory, an operations } \\
\text { shutdown, and likely a take cover. }\end{array}$ \\
\hline W-8 & $\begin{array}{l}\text { Wind damage to } \\
\text { Modified Annex structure } \\
\text { or door }\end{array}$ & High wind & overpressure & $\begin{array}{l}\text { Loaded STS transfer delayed, resulting in heat } \\
\text { up and hydrogen generation leading to } \\
\text { Uncontrolled release of sludge, worker and } \\
\text { public dose. }\end{array}$ & Structure/door qualified to withstand PC-3 wind. & $\begin{array}{l}\text { Administrative control } \\
\text { to shut down transfers } \\
\text { during extreme weather } \\
\text { conditions. }\end{array}$ & $\begin{array}{l}\text { This kind of wind would certainly generate } \\
\text { an adverse weather advisory, an operations } \\
\text { shutdown, and likely a take cover. }\end{array}$ \\
\hline W-9 & $\begin{array}{l}\text { Nitrogen bottle overturns, } \\
\text { knocks off valve }\end{array}$ & High wind & $\begin{array}{l}\text { Spray leak } \\
\text { Splash and splatter } \\
\text { deflagration }\end{array}$ & $\begin{array}{l}\text { Gas bottle missile impacts vital equipment or } \\
\text { hoses, leading to Uncontrolled release of } \\
\text { sludge, worker and public dose. }\end{array}$ & $\begin{array}{l}\text { Bottle rack protected from PC-3 high wind, missile } \\
\text { resistance of lower building walls. }\end{array}$ & None & \\
\hline$\overline{W-10}$ & $\begin{array}{l}\text { Modified Annex } \\
\text { Ventilation equipment } \\
\text { damaged and/or } \\
\text { inoperable }\end{array}$ & $\begin{array}{l}\begin{array}{l}\text { High wind, } \\
\text { missile }\end{array} \\
\end{array}$ & deflagration. & $\begin{array}{l}\text { Impact to duct manifold, stack collapse, } \\
\text { impact to power or control panels, onsite } \\
\text { LOEP lead to loss of STSC active ventilation } \\
\text { and resultant hydrogen buildup, Uncontrolled } \\
\text { release of sludge, worker and public dose. }\end{array}$ & $\begin{array}{l}\text { Structure and ventilation equipment designed to be } \\
\text { protected from PC-3 missile }\end{array}$ & None & \\
\hline W-11 & $\begin{array}{l}\text { Modified Annex } \\
\text { Ventilation filters become } \\
\text { inoperable due to dust } \\
\text { loading }\end{array}$ & High wind & deflagration & $\begin{array}{l}\text { Dust loading plugs inlet and or outlet filters } \\
\text { leading to ventilation inoperability, loss of } \\
\text { active ventilation and Uncontrolled release of } \\
\text { sludge, worker and public dose. }\end{array}$ & $\begin{array}{l}\text { Ventilation filters designed to withstand PC-3 high } \\
\text { wind loading of dust. } \\
\text { Flow or DP monitoring on STSC exhaust. } \\
\text { Inert purge of STSC designed to withstand high } \\
\text { wind. }\end{array}$ & $\begin{array}{l}\text { Administrative control } \\
\text { to purge STSC if } \\
\text { ventilation is lost. }\end{array}$ & \\
\hline W-12 & $\begin{array}{l}\text { Wind missile caused Loss } \\
\text { of pneumatics }\end{array}$ & \begin{tabular}{|l}
$\begin{array}{l}\text { High wind } \\
\text { missile }\end{array}$ \\
\end{tabular} & $\begin{array}{l}\text { LOP evaluated below. } \\
\text { Could be common cause } \\
\text { with other PC-3 } \\
\text { wind/missile hazardous } \\
\text { conditions. }\end{array}$ & $\mathrm{N} / \mathrm{A}$ & PC-3 wind/missile protected pneumatic supply & None & \\
\hline W-13 & Wind initiated LOEP & High wind & $\begin{array}{l}\text { Common cause LOEP-1 } \\
\text { through } 5 \text { and dust loading } \\
\text { or missile caused failure of } \\
\text { backup power leading to } \\
\text { ventilation inoperability. }\end{array}$ & $\begin{array}{l}\text { Hazardous conditions, consequences and } \\
\text { candidate controls as listed under LOEP. } \\
\text { Common cause with wind and missile } \\
\text { addressed under candidate controls listed } \\
\text { here. }\end{array}$ & $\begin{array}{l}\text { Backup power protection from } \mathrm{PC}-3 \text { wind/missile } \\
\text { Inert purge of STSC designed to withstand } \mathrm{PC}-3 \\
\text { high wind and missile. }\end{array}$ & $\begin{array}{l}\text { Administrative } \\
\text { requirement to establish } \\
\text { inert purge following } \\
\text { loss of ventilation. }\end{array}$ & . \\
\hline
\end{tabular}




\begin{tabular}{|c|c|c|c|c|c|c|c|}
\hline \multicolumn{8}{|c|}{ TABLE C-1 "WHAT/IF" HAZARD ANALYSIS STUDY RESULTS - NPH AND EXTERNAL EVENTS } \\
\hline Identifier & "What/If" & Causes & Hazardous Condition & Consequences & $\begin{array}{c}\text { Candidate Controls } \\
\text { Engineered Features }\end{array}$ & Admin Controls & Remarks/Actions \\
\hline $\mathrm{W}-14$ & $\begin{array}{l}\text { Modified Annex structure } \\
\text { collapse leads to fire }\end{array}$ & High wind & overpressure & $\begin{array}{l}\text { Fire initiated by modified annex structural } \\
\text { failure in high wind results in common cause } \\
\text { failure of fire suppression system, fire } \\
\text { involves loaded STSC or STS, Uncontrolled } \\
\text { release of sludge, worker and public dose. }\end{array}$ & $\begin{array}{l}\text { Structure qualified to withstand PC-3 wind } \\
\text { Fire resistant construction } \\
\text { STS design fire resistance }\end{array}$ & $\begin{array}{l}\text { Combustible loading } \\
\text { limits }\end{array}$ & \\
\hline $\mathrm{W}-15$ & $\begin{array}{l}\text { Wind or missile damage } \\
\text { leads to fire }\end{array}$ & $\begin{array}{l}\text { High wind, } \\
\text { missile }\end{array}$ & deflagration & $\begin{array}{l}\text { Fire incapacitates the ventilation system, } \\
\text { leading to hydrogen accumulation and } \\
\text { Uncontrolled release of sludge, worker and } \\
\text { public dose. }\end{array}$ & $\begin{array}{l}\text { Structure and ventilation stack qualified to PC-3 } \\
\text { wind and missile. } \\
\text { Fire resistant construction, } \\
\text { Fire suppression system } \\
\text { Fire area separations. } \\
\text { Wind and missile protected inert purge system. } \\
\end{array}$ & $\begin{array}{l}\text { Combustible loading } \\
\text { limits, } \\
\text { Administrative } \\
\text { requirement to establish } \\
\text { purge following loss of } \\
\text { ventilation. }\end{array}$ & \\
\hline W-16 & $\begin{array}{l}\text { Wind load topples KW } \\
\text { stack }\end{array}$ & High wind & \begin{tabular}{|l|} 
Postulate toppled stack \\
impacts annex or hose barn \\
leading to potential spray or \\
Splash and splatter release \\
\end{tabular} & N/A & None & None & $\begin{array}{l}\text { Stack height evaluated and } 175 \text { ' stack does } \\
\text { not reach to the new Annex facility. }\end{array}$ \\
\hline W-17 & \begin{tabular}{|l} 
Wind load damages \\
Modified Annex structure \\
or roll up door or stack \\
\end{tabular} & High wind & \begin{tabular}{|l} 
Impacts from wind or \\
missile prevent operation \\
pending recovery and repair
\end{tabular} & Operational upset and programmatic delay & None & None & \\
\hline $\mathrm{W}-18$ & \begin{tabular}{|l|} 
Wind load damages \\
Modified Annex structure \\
or roll up door or stack \\
\end{tabular} & High wind & \begin{tabular}{|l} 
Industrial hazard. \\
\end{tabular} & Personnel hazard from structural collapse & None & None & $\begin{array}{l}\text { Structure designed to qualified to } \\
\text { withstand PC-3 wind because of safety } \\
\text { significant }\end{array}$ \\
\hline W-19 & $\begin{array}{l}\text { Wind load damages } \\
\text { Modified Annex structure } \\
\text { leading to collapse onto } \\
\text { transfer lines }\end{array}$ & High Wind & $\begin{array}{l}\text { Spray leak } \\
\text { Splash and splatter release. }\end{array}$ & \begin{tabular}{|l|} 
Transfer lines are impacted by a structure or \\
equipment collapse, dead heading the transfer, \\
decant or overfill recovery pumps, and over \\
pressuring an interfacing service system pipe \\
resulting in an Uncontrolled release of sludge, \\
worker and public dose. \\
\end{tabular} & $\begin{array}{l}\text { PC-3 Wind resistant design of structure. } \\
\text { Relieve valves (rupture discs) on transfer line. } \\
\text { Interfacing system block/check valves to prevent } \\
\text { backflow. }\end{array}$ & $\begin{array}{l}\text { Administrative control } \\
\text { to shut down transfers } \\
\text { during extreme weather } \\
\text { conditions. }\end{array}$ & \\
\hline $\mathrm{W}-20$ & $\begin{array}{l}\text { Wind catches door and } \\
\text { forces it to extreme }\end{array}$ & High Wind & Industrial hazard. & $\begin{array}{l}\text { Personnel door is forced open and broken, or } \\
\text { opened or closed impacting personnel during } \\
\text { operation. Personnel safety impact and } \\
\text { potential operational upset. }\end{array}$ & None & None & $\begin{array}{l}\text { Ensure that door closer resists wind } \\
\text { effects. }\end{array}$ \\
\hline \multicolumn{8}{|l|}{ FLOOD } \\
\hline FL-1 & $\begin{array}{l}\text { Flood damage prevents } \\
\text { STS Transport }\end{array}$ & Flood & overpressure & $\begin{array}{l}\text { Water line failure floods/undermines road and } \\
\text { prevents STS transport leading to } \\
\text { Uncontrolled release of sludge, worker and } \\
\text { public dose. }\end{array}$ & STS vent and purge ports, port tools & $\begin{array}{l}\text { Administrative } \\
\text { requirement to monitor } \\
\text { pressure and vent STS } \\
\text { when delayed. } \\
\end{array}$ & $\begin{array}{l}\text { Facility above Columbia river PC-3 flood } \\
\text { level }\end{array}$ \\
\hline FL-2 & $\begin{array}{l}\text { Flood damages load cells, } \\
\text { STS }\end{array}$ & Flood & $\begin{array}{l}\text { Accidental sprinkler system } \\
\text { actuation leads to flood of } \\
\text { truck bay sump and impact } \\
\text { to load cell instrumentation, } \\
\text { or flooding of STS. } \\
\end{array}$ & $\begin{array}{l}\text { Operational upset, programmatic delay for } \\
\text { recovery }\end{array}$ & None & None & \\
\hline
\end{tabular}


TABLE C-1 "WHAT/IF" HAZARD ANALYSIS STUDY RESULTS - NPH AND EXTERNAL EVENTS

\begin{tabular}{|c|c|c|c|c|c|c|c|}
\hline Identifier & "What/If" & Causes & Hazardous Condition & Consequences & $\begin{array}{l}\text { Candidate Controls } \\
\text { Engineered Features }\end{array}$ & Admin Controls & Remarks/Actions \\
\hline FL-3 & $\begin{array}{l}\text { Flood damages } \\
\text { ventilation }\end{array}$ & Flood & deflagration & $\begin{array}{l}\text { Accidental sprinkler actuation impacts } \\
\text { ventilation controls incapacitating system } \\
\text { operability with resulting loss of active } \\
\text { ventilation, hydrogen accumulation and } \\
\text { Uncontrolled release of sludge, worker and } \\
\text { public dose. }\end{array}$ & $\begin{array}{l}\text { Protect ventilation system from impact by sprinkler } \\
\text { system actuation. } \\
\text { Provide capability to purge STSC if ventilation is } \\
\text { lost }\end{array}$ & $\begin{array}{l}\text { Administrative control } \\
\text { to purge STSC if } \\
\text { ventilation flow is lost. }\end{array}$ & \\
\hline \multicolumn{8}{|l|}{ SNOW } \\
\hline SF-1 & $\begin{array}{l}\text { Snow load fails Modified } \\
\text { Annex structure during } \\
\text { transfer, decant or overfill } \\
\text { recovery }\end{array}$ & Snowfall & $\begin{array}{l}\text { Spray leak } \\
\text { Splash and splatter release. }\end{array}$ & $\begin{array}{l}\text { Structure failure impacts transfer lines, } \\
\text { leading to Uncontrolled release of sludge, } \\
\text { worker and public dose. }\end{array}$ & Design of structure to withstand snowfall & $\begin{array}{l}\text { Administrative control } \\
\text { to shut down transfers } \\
\text { during extreme weather } \\
\text { conditions. } \\
\end{array}$ & \\
\hline SF-2 & $\begin{array}{l}\text { Snow load fails Modified } \\
\text { Annex structure }\end{array}$ & Snowfall & deflagration & $\begin{array}{l}\text { Structure failure impacts ventilation system } \\
\text { and prevents STSC active ventilation leading } \\
\text { to hydrogen buildup and Uncontrolled release } \\
\text { of sludge, worker and public dose. }\end{array}$ & $\begin{array}{l}\text { Design of structure to withstand snowfall, } \\
\text { inert gas purge capability }\end{array}$ & $\begin{array}{l}\text { Administrative control } \\
\text { to purge STSC if } \\
\text { ventilation flow is lost. }\end{array}$ & May not be able to access to install purge \\
\hline SF-3 & $\begin{array}{l}\text { Snow load fails Modified } \\
\text { Annex structure }\end{array}$ & Snowfall & overpressure & $\begin{array}{l}\text { Structure failure prevents loaded STS from } \\
\text { departing resulting in hydrogen gas } \\
\text { generation, and Uncontrolled release of } \\
\text { sludge, worker and public dose. }\end{array}$ & $\begin{array}{l}\text { Design of structure to withstand snowfall, } \\
\text { STS vent and purge ports, port tools }\end{array}$ & $\begin{array}{l}\text { Administrative } \\
\text { requirement to monitor } \\
\text { pressure and vent STS } \\
\text { when delayed. }\end{array}$ & \\
\hline SF-4 & Snowfall induced LOEP & Snowfall & See LOEP below & $\mathrm{N} / \mathrm{A}$ & N/A & $\mathrm{N} / \mathrm{A}$ & \\
\hline SF-5 & Snowfall induced LOP & Snowfall & $\begin{array}{l}\text { Structure collapse in } \\
\text { mechanical room or } \\
\text { elsewhere impacting } \\
\text { instrument air. See LOP } \\
\text { below under FF-6 }\end{array}$ & See LOP below under FF- 6 & N/A & N/A & \\
\hline SF-6 & $\begin{array}{l}\text { Snowfall prevents } \\
\text { transport to T Plant }\end{array}$ & Snowfall & overpressure & $\begin{array}{l}\text { Road closure/transportation restriction } \\
\text { prevents transport of STS, leading to build up } \\
\text { of hydrogen and Uncontrolled release of } \\
\text { sludge, worker and public dose. }\end{array}$ & STS vent and purge ports, port tools & $\begin{array}{l}\text { Administrative } \\
\text { requirement to monitor } \\
\text { pressure and vent STS } \\
\text { when delayed. }\end{array}$ & \\
\hline SF-7 & $\begin{array}{l}\text { Modified Annex Roof } \\
\text { failure and loss of } \\
\text { confinement }\end{array}$ & Snowfall & $\begin{array}{l}\text { Loss of confinement } \\
\text { integrity, and upset to } \\
\text { ventilation system }\end{array}$ & Operational upset & N/A & N/A & \\
\hline SF-8 & $\begin{array}{l}\text { Modified Annex Roof } \\
\text { failure or ice dam and } \\
\text { water inleakge }\end{array}$ & Snowfall & $\begin{array}{l}\text { Water inleakge impacts } \\
\text { electrical panels failing } \\
\text { instrumentation, switchgear, } \\
\text { interlocks }\end{array}$ & Operational upset and programmatic delay & N/A & N/A & \\
\hline SF-9 & $\begin{array}{l}\text { Snowfall fails Modified } \\
\text { Annex structure and } \\
\text { impacts transfer lines }\end{array}$ & Snowfall & $\begin{array}{l}\text { Spray leak } \\
\text { Splash and splatter release. }\end{array}$ & $\begin{array}{l}\text { Transfer lines are impacted by a structure or } \\
\text { equipment collapse, dead heading the transfer, } \\
\text { decant, or overfill recovery pumps, and over } \\
\text { pressuring an interfacing service system pipe } \\
\text { resulting in an Uncontrolled release of sludge, } \\
\text { worker and public dose. }\end{array}$ & $\begin{array}{l}\text { Design of structure to withstand snowfall, } \\
\text { Block/check valves protecting interfacing lines }\end{array}$ & None & \\
\hline SF-10 & $\begin{array}{l}\text { Snowfall fails Modified } \\
\text { Annex structure }\end{array}$ & Snowfall & Industrial Safety & Personnel hazard from structural collapse & None & None & \\
\hline
\end{tabular}




\begin{tabular}{|c|c|c|c|c|c|c|c|}
\hline \multicolumn{8}{|c|}{ TABLE C-1 "WHAT/IF" HAZARD ANALYSIS STUDY RESULTS - NPH AND EXTERNAL EVENTS } \\
\hline Identifier & "What/If" & Causes & Hazardous Condition & Consequences & $\begin{array}{c}\text { Candidate Controls } \\
\text { Engineered Features }\end{array}$ & Admin Controls & Remarks/Actions \\
\hline SF-11 & $\begin{array}{l}\text { Snowfall blocks Modified } \\
\text { Annex door closed }\end{array}$ & Snowfall & Industrial safety & $\begin{array}{l}\text { Snowfall blocked door impacts egress paths } \\
\text { and elevated platform life safety }\end{array}$ & Protect doorway from snow accumulation & $\begin{array}{l}\text { Provide check of access } \\
\text { prior to occupancy } \\
\text { during snowfall periods } \\
\end{array}$ & \\
\hline SF-12 & $\begin{array}{l}\text { Modified Annex Roof } \\
\text { failure or ice dam and } \\
\text { water inleakge }\end{array}$ & Snowfall & deflagration & $\begin{array}{l}\text { Water inleakge impacts HVAC Controls and } \\
\text { prevents STSC active ventilation leading to } \\
\text { hydrogen buildup and Uncontrolled release of } \\
\text { sludge, worker and public dose. }\end{array}$ & $\begin{array}{l}\text { Design of structure to withstand snowfall, } \\
\text { inert gas purge capability }\end{array}$ & $\begin{array}{l}\text { Administrative control } \\
\text { to purge STSC if } \\
\text { ventilation flow is lost. }\end{array}$ & \\
\hline \multicolumn{8}{|c|}{ ASHFALL } \\
\hline AF-1 & $\begin{array}{l}\text { Ashfall load fails } \\
\text { Modified Annex structure } \\
\text { during transfer, decant or } \\
\text { overfill recovery }\end{array}$ & Ashfall & $\begin{array}{l}\text { Spray leak } \\
\text { Splash and splatter release. }\end{array}$ & $\begin{array}{l}\text { Structure failure impacts transfer lines, } \\
\text { leading to Uncontrolled release of sludge, } \\
\text { worker and public dose. }\end{array}$ & Design of structure to withstand snowfall & $\begin{array}{l}\text { Administrative control } \\
\text { to shut down transfers } \\
\text { during extreme weather } \\
\text { conditions. }\end{array}$ & \\
\hline AF-2 & $\begin{array}{l}\text { Ashfall load fails } \\
\text { Modified Annex structure }\end{array}$ & Ashfall & deflagration & $\begin{array}{l}\text { Structure failure impacts ventilation system } \\
\text { and prevents STSC active ventilation leading } \\
\text { to hydrogen buildup and Uncontrolled release } \\
\text { of sludge, worker and public dose. }\end{array}$ & $\begin{array}{l}\text { Design of structure to withstand snowfall, } \\
\text { inert gas purge capability }\end{array}$ & $\begin{array}{l}\text { Administrative control } \\
\text { to purge STSC if } \\
\text { ventilation flow is lost. }\end{array}$ & May not be able to access to install purge \\
\hline AF-3 & $\begin{array}{l}\text { Ashfall load fails } \\
\text { Modified Annex structure }\end{array}$ & Ashfall & overpressure & $\begin{array}{l}\text { Structure failure prevents loaded STS from } \\
\text { departing resulting in hydrogen gas } \\
\text { generation, and Uncontrolled release of } \\
\text { sludge, worker and public dose. }\end{array}$ & $\begin{array}{l}\text { Design of structure to withstand snowfall, } \\
\text { STS vent and purge ports, port tools }\end{array}$ & $\begin{array}{l}\text { Administrative } \\
\text { requirement to monitor } \\
\text { pressure and vent STS } \\
\text { when delayed. } \\
\end{array}$ & \\
\hline $\mathrm{AF}-4$ & Ashfall induced LOEP & Ashfall & $\begin{array}{l}\text { See LOEP- } 1 \text { through } 5 \text { for } \\
\text { conditions, consequences } \\
\text { and candidate controls }\end{array}$ & N/A & N/A & N/A & \\
\hline AF-5 & Ashfall induced LOP & Ashfall & See LOP below & N/A & N/A & N/A & \\
\hline AF-6 & $\begin{array}{l}\text { Ashfall prevents transport } \\
\text { to T Plant }\end{array}$ & Ashfall & overpressure & $\begin{array}{l}\text { Road closure/transportation restriction } \\
\text { prevents transport of STS, leading to build up } \\
\text { of hydrogen and Uncontrolled release of } \\
\text { sludge, worker and public dose. }\end{array}$ & STS vent and purge ports, port tools & $\begin{array}{l}\text { Administrative } \\
\text { requirement to monitor } \\
\text { pressure and vent STS } \\
\text { when delayed. }\end{array}$ & \\
\hline AF-7 & $\begin{array}{l}\text { Modified Annex Roof } \\
\text { failure and loss of } \\
\text { confinement }\end{array}$ & Ashfall & $\begin{array}{l}\text { Loss of confinement } \\
\text { integrity, and upset to } \\
\text { ventilation system }\end{array}$ & Operational upset & N/A & N/A & \\
\hline AF-8 & $\begin{array}{l}\text { Modified Annex Roof } \\
\text { failure and subsequent } \\
\text { rainfall }\end{array}$ & Ashfall & $\begin{array}{l}\text { Water inleakge impacts } \\
\text { electrical panels failing } \\
\text { instrumentation, switchgear, } \\
\text { interlocks }\end{array}$ & Operational upset and programmatic delay & N/A & N/A & \\
\hline AF-9 & $\begin{array}{l}\text { Ashfall fails Modified } \\
\text { Annex structure and } \\
\text { impacts transfer lines }\end{array}$ & Ashfall & $\begin{array}{l}\text { Spray leak } \\
\text { Splash and splatter release. }\end{array}$ & $\begin{array}{l}\text { Transfer lines are impacted by a structure or } \\
\text { equipment collapse, dead heading the transfer, } \\
\text { decant, or overfill recovery pumps, and over } \\
\text { pressuring an interfacing service system pipe } \\
\text { resulting in an Uncontrolled release of sludge, } \\
\text { worker and public dose. }\end{array}$ & $\begin{array}{l}\text { Design of structure to withstand snowfall, } \\
\text { Block/check valves protecting interfacing lines }\end{array}$ & None & \\
\hline AF-10 & $\begin{array}{l}\text { Ashfall fails Modified } \\
\text { Annex structure }\end{array}$ & Ashfall & Industrial Safety & Personnel hazard from structural collapse & None & None & \\
\hline
\end{tabular}




\begin{tabular}{|c|c|c|c|c|c|c|c|}
\hline \multicolumn{8}{|c|}{ TABLE C-1 “WHAT/IF" HAZARD ANALYSIS STUDY RESULTS - NPH AND EXTERNAL EVENTS } \\
\hline Identifier & "What/If" & Causes & Hazardous Condition & Consequences & $\begin{array}{l}\text { Candidate Controls } \\
\text { Engineered Features }\end{array}$ & Admin Controls & Remarks/Actions \\
\hline AF-11 & $\begin{array}{l}\text { Ashfall blocks Modified } \\
\text { Annex door closed }\end{array}$ & Ashfall & Industrial safety & $\begin{array}{l}\text { Ashfall blocked door impacts egress paths and } \\
\text { elevated platform life safety }\end{array}$ & None & None & \\
\hline AF-12 & $\begin{array}{l}\text { Modified Annex } \\
\text { Ventilation filters become } \\
\text { inoperable due to ash }\end{array}$ & Ashfall & deflagration & $\begin{array}{l}\text { Uncontrolled release of sludge, worker and } \\
\text { public dose. }\end{array}$ & $\begin{array}{l}\text { Ventilation filters designed to withstand ashfall } \\
\text { loading of dust. } \\
\text { Flow or DP monitoring on STSC exhaust. } \\
\text { Inert purge of STSC designed to withstand ashfall. }\end{array}$ & $\begin{array}{l}\text { Administrative control } \\
\text { to purge STSC if } \\
\text { ventilation is lost. }\end{array}$ & \\
\hline \multicolumn{8}{|l|}{ RAIN } \\
\hline R-1 & $\begin{array}{l}\text { Heavy rainfall delays } \\
\text { transport }\end{array}$ & $\begin{array}{l}\text { Heavy } \\
\text { rainfall }\end{array}$ & overpressure & $\begin{array}{l}\text { Delay in transportation of sealed cask leads to } \\
\text { heat-up and hydrogen pressurization of STS, } \\
\text { with eventual Uncontrolled release of sludge, } \\
\text { worker and public dose. }\end{array}$ & STS vent and purge ports, port tools & $\begin{array}{l}\text { Administrative } \\
\text { requirement to monitor } \\
\text { pressure and vent STS } \\
\text { when delayed. }\end{array}$ & \\
\hline $\mathrm{R}-2$ & $\begin{array}{l}\text { Heavy rainfall and winds } \\
\text { result in wetting of } \\
\text { electrical equipment }\end{array}$ & $\begin{array}{l}\text { Heavy } \\
\text { rainfall }\end{array}$ & deflagration & $\begin{array}{l}\text { Wet electrical components cause failure of } \\
\text { ventilation system, leading to buildup of } \\
\text { hydrogen in STSC and Uncontrolled release } \\
\text { of sludge, worker and public dose. }\end{array}$ & $\begin{array}{l}\text { Structure design to maintain operation in heavy } \\
\text { rainfall, } \\
\text { STS vent and purge ports, port tools }\end{array}$ & $\begin{array}{l}\text { Administrative } \\
\text { requirement to monitor } \\
\text { pressure and vent STS } \\
\text { when delayed. }\end{array}$ & \\
\hline R-3 & $\begin{array}{l}\text { Heavy rainfall leads to } \\
\text { LOEP }\end{array}$ & \begin{tabular}{|l|}
$\begin{array}{l}\text { Heavy } \\
\text { rainfall }\end{array}$ \\
\end{tabular} & $\begin{array}{l}\text { LOEP related hazardous } \\
\text { conditions listed below }\end{array}$ & $\begin{array}{l}\text { See LOEP-1 through } 5 \text { conditions, } \\
\text { consequences and candidate controls. }\end{array}$ & $\mathrm{N} / \mathrm{A}$ & N/A & \\
\hline \multicolumn{8}{|c|}{ LIGHTNING } \\
\hline $\mathrm{L}-1$ & \begin{tabular}{|l|} 
Initiates LOEP \\
\end{tabular} & \begin{tabular}{|l|} 
Lightning \\
\end{tabular} & $\begin{array}{l}\text { LOEP related hazardous } \\
\text { conditions listed below }\end{array}$ & $\begin{array}{l}\text { See LOEP-1 through } 5 \text { conditions, } \\
\text { consequences and candidate controls. }\end{array}$ & N/A & $\mathrm{N} / \mathrm{A}$ & \\
\hline$\overline{\mathrm{L}-2}$ & $\begin{array}{l}\text { Lightning damages } \\
\text { Modified Annex HVAC } \\
\text { switchgear or control } \\
\text { system }\end{array}$ & Lightning & deflagration & $\begin{array}{l}\text { Loss of ventilation flow to loaded STSC leads } \\
\text { to hydrogen buildup, and Uncontrolled release } \\
\text { of sludge, worker and public dose. }\end{array}$ & \begin{tabular}{|l|} 
Lightning protection of structure, \\
Lightning protection of inert gas purge equipment
\end{tabular} & $\begin{array}{l}\text { Administrative control } \\
\text { to purge STSC if } \\
\text { ventilation is lost. }\end{array}$ & \\
\hline $\mathrm{L}-3$ & $\begin{array}{l}\text { Lightning initiates a fire } \\
\text { resulting in any of the } \\
\text { hazardous conditions } \\
\text { under Fire below. }\end{array}$ & Lightning & $\begin{array}{l}\text { Fire related hazardous } \\
\text { condition listed below }\end{array}$ & $\begin{array}{l}\text { Process upset and Uncontrolled release of } \\
\text { sludge, worker and public dose. }\end{array}$ & $\begin{array}{l}\text { Lightning protection of structure, } \\
\text { Fire protection features listed in events below. }\end{array}$ & None & \\
\hline $\mathrm{L}-4$ & $\begin{array}{l}\text { Transport of loaded STS } \\
\text { delayed by lightning }\end{array}$ & Lightning & overpressure & $\begin{array}{l}\text { Gas accumulation and Uncontrolled release of } \\
\text { sludge, worker and public dose. }\end{array}$ & $\begin{array}{l}\text { Lightning protection of structure, } \\
\text { STS vent and purge ports, port tools }\end{array}$ & $\begin{array}{l}\text { Administrative } \\
\text { requirement to monitor } \\
\text { pressure and vent STS } \\
\text { when delayed. }\end{array}$ & \\
\hline L-5 & $\begin{array}{l}\text { Lightning damages } \\
\text { interlocks or controls }\end{array}$ & Lightning & Splash and splatter & $\begin{array}{l}\text { Lightning strike makes interlocks for process } \\
\text { inoperable leading to overfill and leak, dose to } \\
\text { worker and public. Operational upset and } \\
\text { TSR Operability issue }\end{array}$ & $\begin{array}{l}\text { Lightning protection for structure and circuitry. } \\
\text { Fail-safe design of interlocks }\end{array}$ & $\begin{array}{l}\text { Operability checks prior } \\
\text { to operation, } \\
\text { Operations restrictions } \\
\text { during lightning storms } \\
\end{array}$ & \\
\hline \multicolumn{8}{|c|}{ HIGH AMBIENT TEMPERATURE } \\
\hline HAT-1 & $\begin{array}{l}\text { Heat impacts shipping } \\
\text { window }\end{array}$ & $\begin{array}{l}\text { High ambient } \\
\text { temperature }\end{array}$ & overpressure & $\begin{array}{l}\text { Gas accumulation and Uncontrolled release of } \\
\text { sludge, worker and public dose. }\end{array}$ & $\begin{array}{l}\text { Design of STSC and Cask to accommodate high } \\
\text { ambient temperature as part of shipping window } \\
\text { analysis }\end{array}$ & None & \\
\hline
\end{tabular}




\begin{tabular}{|c|c|c|c|c|c|c|c|}
\hline \multicolumn{8}{|c|}{ TABLE C-1 "WHAT/IF" HAZARD ANALYSIS STUDY RESULTS - NPH AND EXTERNAL EVENTS } \\
\hline Identifier & "What/If" & Causes & Hazardous Condition & Consequences & $\begin{array}{c}\text { Candidate Controls } \\
\text { Engineered Features }\end{array}$ & Admin Controls & Remarks/Actions \\
\hline LAT-1 & $\begin{array}{l}\text { Freezing conditions } \\
\text { impact shipping window }\end{array}$ & $\begin{array}{l}\text { Low ambient } \\
\text { temperature }\end{array}$ & overpressure & $\begin{array}{l}\text { Roll-up door inoperability or road closure } \\
\text { delays shipping leading to gas accumulation } \\
\text { and Uncontrolled release of sludge, worker } \\
\text { and public dose. }\end{array}$ & $\begin{array}{l}\text { Design of door ensures operability in freezing } \\
\text { conditions; } \\
\text { STS vent and purge ports, port tools }\end{array}$ & $\begin{array}{l}\text { Administrative } \\
\text { requirement to monitor } \\
\text { pressure and vent STS } \\
\text { when delayed. }\end{array}$ & \\
\hline LAT-2 & $\begin{array}{l}\text { Freezing conditions } \\
\text { impact shipping }\end{array}$ & $\begin{array}{l}\text { Low ambient } \\
\text { temperature }\end{array}$ & $\begin{array}{l}\text { STSC freezes during delay } \\
\text { on road, resulting in damage } \\
\text { to vessel. }\end{array}$ & Operational upset. & None & $\begin{array}{l}\text { Transport times and } \\
\text { limits on transport } \\
\text { during extreme weather. } \\
\text { Emergency response } \\
\text { procedures } \\
\end{array}$ & \\
\hline LAT-3 & $\begin{array}{l}\text { Low temperatures freeze } \\
\text { liquid in transfer line } \\
\text { resulting in transfer line } \\
\text { failure. }\end{array}$ & $\begin{array}{l}\text { Low ambient } \\
\text { temperature }\end{array}$ & $\begin{array}{l}\text { Inner hose low temperature } \\
\text { freezes, leaking liquid and } \\
\text { prevents leak detection. }\end{array}$ & $\begin{array}{l}\text { Operational upset, perhaps leading to } \\
\text { additional failure. }\end{array}$ & $\begin{array}{l}\text { Freeze damage resistance of hose, } \\
\text { Freeze protection for leak detection } \\
\text { Freeze protection for transfer line }\end{array}$ & $\begin{array}{l}\text { Administrative control } \\
\text { to shut down transfers } \\
\text { during extreme weather } \\
\text { conditions. }\end{array}$ & \\
\hline LAT-4 & $\begin{array}{l}\text { Freezing condition } \\
\text { impacts STSC vent }\end{array}$ & $\begin{array}{l}\text { Low ambient } \\
\text { temperature }\end{array}$ & deflagration. & $\begin{array}{l}\text { Moisture separator freezes preventing gas } \\
\text { flow. Buildup of hydrogen leading to } \\
\text { Uncontrolled release of sludge, worker and } \\
\text { public dose. }\end{array}$ & $\begin{array}{l}\text { New annex HVAC conditioning capability, } \\
\text { freeze protected inert gas purge system }\end{array}$ & $\begin{array}{l}\text { Administrative control } \\
\text { to purge STSC if } \\
\text { ventilation is lost. }\end{array}$ & \\
\hline LAT-5 & $\begin{array}{l}\text { Fire initiates during } \\
\text { freezing condition which } \\
\text { had frozen Modified } \\
\text { Annex sprinkler system, }\end{array}$ & $\begin{array}{l}\text { Low ambient } \\
\text { temperature }\end{array}$ & $\begin{array}{l}\text { deflagration } \\
\text { Spray leak } \\
\text { Splash and splatter. }\end{array}$ & $\begin{array}{l}\text { Operational upset. Loss of safety SSC, } \\
\text { potential for unmitigated fire. Unmitigated } \\
\text { fire leads to loss of ventilation, leak and } \\
\text { potential for Uncontrolled release of sludge, } \\
\text { worker and public dose. }\end{array}$ & $\begin{array}{l}\text { New annex HVAC conditioning capability } \\
\text { Design of sprinkler system to prevent freeze }\end{array}$ & $\begin{array}{l}\text { Administrative control } \\
\text { to shut down or preclude } \\
\text { transfers if temp below } \\
\text { some value. } \\
\text { Administrative control } \\
\text { to purge STSC if } \\
\text { ventilation is lost. }\end{array}$ & \\
\hline LAT-6 & $\begin{array}{l}\text { Freezing Condition holds } \\
\text { external door closed }\end{array}$ & $\begin{array}{l}\text { Low ambient } \\
\text { temperature }\end{array}$ & Industrial hazard & $\begin{array}{l}\text { Frozen door jamb or lock prevents egress or } \\
\text { access Life safety code issue, operational } \\
\text { upset }\end{array}$ & None & None & \\
\hline \multicolumn{8}{|c|}{ LOSS OF ELECTRICAL POWER } \\
\hline LOEP-1 & $\begin{array}{l}\text { Loss of power during } \\
\text { transfer or overfill } \\
\text { recovery }\end{array}$ & LOEP & $\begin{array}{l}\text { Spray leak } \\
\text { Splash and splatter. }\end{array}$ & $\begin{array}{l}\text { Sludge settles in line, or valve closes resulting } \\
\text { in blocked line on restart and system } \\
\text { overpressure with attendant uncontrolled } \\
\text { release of sludge, worker and public dose. }\end{array}$ & $\begin{array}{l}\text { Bladder tank flush of transfer line. Line design } \\
\text { pressure prevents deadhead failure. }\end{array}$ & $\begin{array}{l}\text { Emergency response } \\
\text { program to control } \\
\text { recovery }\end{array}$ & $\begin{array}{l}\text { Valves are FL (fail as is) in transfer line. } \\
\text { LOEP may reasonably result in LOP and } \\
\text { LOW }\end{array}$ \\
\hline LOEP-2 & $\begin{array}{l}\text { Loss of power to } \\
\text { ventilation system when } \\
\text { STSC is loaded }\end{array}$ & LOEP & deflagration. & $\begin{array}{l}\text { Accumulation of hydrogen leading to } \\
\text { uncontrolled release of sludge, worker and } \\
\text { public dose. }\end{array}$ & $\begin{array}{l}\text { Backup power to ventilation system, } \\
\text { Inert gas purge capability }\end{array}$ & $\begin{array}{l}\text { Administrative } \\
\text { requirement to establish } \\
\text { purge following loss of } \\
\text { ventilation. }\end{array}$ & \\
\hline LOEP-3 & $\begin{array}{l}\text { Loss of power when STS } \\
\text { loaded. }\end{array}$ & LOEP & overpressure of STS. & $\begin{array}{l}\text { Closed roll-up door prevents departure, } \\
\text { leading to gas accumulation and uncontrolled } \\
\text { release of sludge, worker and public dose. }\end{array}$ & $\begin{array}{l}\text { Backup power to door, } \\
\text { STS vent and purge ports, } \\
\text { port tools, } \\
\text { manual inert gas purge capability }\end{array}$ & $\begin{array}{l}\text { Administrative } \\
\text { requirement to monitor } \\
\text { pressure and vent STS } \\
\text { when delayed. }\end{array}$ & \\
\hline
\end{tabular}




\begin{tabular}{|c|c|c|c|c|c|c|c|}
\hline \multicolumn{8}{|c|}{ TABLE C-1 “WHAT/IF” HAZARD ANALYSIS STUDY RESULTS - NPH AND EXTERNAL EVENTS } \\
\hline Identifier & "What/If" & Causes & Hazardous Condition & Consequences & $\begin{array}{l}\text { Candidate Controls } \\
\text { Engineered Features }\end{array}$ & Admin Controls & Remarks/Actions \\
\hline LOEP-4 & $\begin{array}{l}\text { Loss of power when } \\
\text { closing STS }\end{array}$ & LOEP & deflagration. & $\begin{array}{l}\text { Stuck or dropped STS lid causes inability to } \\
\text { purge or ventilate leading to hydrogen } \\
\text { accumulation and uncontrolled release of } \\
\text { sludge, worker and public dose. }\end{array}$ & Backup power to crane to clear and then purge. & & \\
\hline LOEP-5 & $\begin{array}{l}\text { Loss of power during } \\
\text { cold weather }\end{array}$ & LOEP & $\begin{array}{l}\text { Freeze of lines, or liquid in } \\
\text { STSC damaging equipment }\end{array}$ & Operational upset & None & None & \\
\hline \multicolumn{8}{|c|}{ RANGE FIRE } \\
\hline RF-1 & $\begin{array}{l}\text { Delays departure of } \\
\text { loaded STS }\end{array}$ & Range Fire & overpressure & $\begin{array}{l}\text { Range fire prevents departure, leading to gas } \\
\text { accumulation and Uncontrolled release of } \\
\text { sludge, worker and public dose. }\end{array}$ & STS vent and purge ports, port tools & $\begin{array}{l}\text { Administrative } \\
\text { requirement to monitor } \\
\text { pressure and vent STS } \\
\text { when delayed. }\end{array}$ & \\
\hline RF-2 & $\begin{array}{l}\text { Initiates Modified Annex } \\
\text { facility fire }\end{array}$ & Range Fire & $\begin{array}{l}\text { Range fire leads to facility } \\
\text { fire and attendant hazardous } \\
\text { conditions }\end{array}$ & $\begin{array}{l}\text { See Facility Fire FF-1 through } 5 \text { hazardous } \\
\text { conditions, consequences and candidate } \\
\text { controls. }\end{array}$ & N/A & N/A & \\
\hline RF-3 & Initiates a LOEP & Range Fire & $\begin{array}{l}\text { Range fire leads to LOEP } \\
\text { and attendant hazardous } \\
\text { conditions }\end{array}$ & $\begin{array}{l}\text { See LOEP-1 through } 5 \text { for hazardous } \\
\text { conditions, consequences and candidate } \\
\text { controls }\end{array}$ & N/A & N/A & \\
\hline $\mathrm{RF}-4$ & $\begin{array}{l}\text { Modified Annex } \\
\text { Structure failure }\end{array}$ & Range Fire & \begin{tabular}{|l|} 
deflagration \\
STSC overturn, spill
\end{tabular} & $\begin{array}{l}\text { Range fire fails structure and prevents purge } \\
\text { of STSC or overturns STSC leading to } \\
\text { Uncontrolled release of sludge, worker and } \\
\text { public dose. }\end{array}$ & $\begin{array}{l}\text { Fire resistant structure and components, } \\
\text { Fire resistance of inert gas purge }\end{array}$ & $\begin{array}{l}\text { Administrative control } \\
\text { to purge STSC if } \\
\text { ventilation is lost. }\end{array}$ & \\
\hline RF-5 & $\begin{array}{l}\text { Modified Annex } \\
\text { Structure failure }\end{array}$ & Range Fire & Industrial hazard & Personnel hazard from structural collapse & None & None & \\
\hline $\mathrm{RF}-6$ & $\begin{array}{l}\text { Fire smoke and soot plugs } \\
\text { HVAC filters }\end{array}$ & Range Fire & deflagration & $\begin{array}{l}\text { Smoke and soot plugs HVAC, preventing } \\
\text { ventilation of the STSC, buildup of hydrogen } \\
\text { and Uncontrolled release of sludge, worker } \\
\text { and public dose. }\end{array}$ & $\begin{array}{l}\text { HVAC design that accommodates or mitigates } \\
\text { range fire smoke, } \\
\text { STSC inerting system that functions during and } \\
\text { after range fire }\end{array}$ & $\begin{array}{l}\text { Administrative } \\
\text { requirement to establish } \\
\text { purge if ventilation is } \\
\text { lost }\end{array}$ & \\
\hline \multicolumn{8}{|c|}{ VEHICLE IMPACT } \\
\hline VI-1 & $\begin{array}{l}\text { Modified Annex } \\
\text { Structure failure while } \\
\text { backing trailer in }\end{array}$ & \begin{tabular}{|l} 
Vehicle \\
Impact
\end{tabular} & Industrial hazard & $\begin{array}{l}\text { Annex structure damaged while placing trailer } \\
\text { in bay. Operational upset, programmatic delay }\end{array}$ & None & None & \\
\hline VI-2 & $\begin{array}{l}\text { Vehicle impacts Modified } \\
\text { Annex structure while } \\
\text { loading STSC or overfill } \\
\text { recovery }\end{array}$ & $\begin{array}{l}\text { Vehicle } \\
\text { impact }\end{array}$ & $\begin{array}{l}\text { Spray leak } \\
\text { Splash and splatter leak }\end{array}$ & $\begin{array}{l}\text { Vehicle impact to structure results in transfer } \\
\text { line failure and Uncontrolled release of } \\
\text { sludge, worker and public dose. }\end{array}$ & Vehicle barriers around structure & $\begin{array}{l}\text { Administrative traffic } \\
\text { restrictions. }\end{array}$ & \\
\hline
\end{tabular}




\begin{tabular}{|c|c|c|c|c|c|c|c|}
\hline \multicolumn{8}{|c|}{ TABLE C-1 “WHAT/IF" HAZARD ANALYSIS STUDY RESULTS - NPH AND EXTERNAL EVENTS } \\
\hline Identifier & "What/If" & Causes & Hazardous Condition & Consequences & $\begin{array}{c}\text { Candidate Controls } \\
\text { Engineered Features }\end{array}$ & Admin Controls & Remarks/Actions \\
\hline VI-3 & $\begin{array}{l}\text { Vehicle Impacts Modified } \\
\text { Annex structure with } \\
\text { loaded STS inside }\end{array}$ & $\begin{array}{l}\text { Vehicle } \\
\text { impact }\end{array}$ & overpressure & $\begin{array}{l}\text { Vehicle impact prevents loaded STS transport } \\
\text { with gas accumulation and Uncontrolled } \\
\text { release of sludge, worker and public dose. }\end{array}$ & $\begin{array}{l}\text { Vehicle barriers around structure } \\
\text { STS vent and purge ports, port tools } \\
\text { Inert gas purge capability }\end{array}$ & $\begin{array}{l}\text { Administrative traffic } \\
\text { restrictions } \\
\text { Administrative } \\
\text { requirement to monitor } \\
\text { pressure and vent STS } \\
\text { when delayed. }\end{array}$ & \\
\hline VI-4 & $\begin{array}{l}\text { Vehicle impact causes } \\
\text { LOEP }\end{array}$ & $\begin{array}{l}\text { Vehicle } \\
\text { impact }\end{array}$ & $\begin{array}{l}\text { Vehicle impact leads to } \\
\text { LOEP and attendant } \\
\text { hazardous conditions }\end{array}$ & $\begin{array}{l}\text { See LOEP-1 through } 5 \text { for hazardous } \\
\text { conditions, consequences and candidate } \\
\text { controls }\end{array}$ & N/A & N/A & \\
\hline VI-5 & $\begin{array}{l}\text { Vehicle impact causes } \\
\text { facility fire }\end{array}$ & \begin{tabular}{|l|} 
Vehicle \\
impact
\end{tabular} & $\begin{array}{l}\text { Vehicle impact leads to } \\
\text { facility fire and attendant } \\
\text { hazardous conditions }\end{array}$ & $\begin{array}{l}\text { See Facility fire FF-1 through } 5 \text { for hazardous } \\
\text { conditions, consequences and candidate } \\
\text { controls }\end{array}$ & Vehicle barriers around structure & $\begin{array}{l}\text { Administrative traffic } \\
\text { restrictions. }\end{array}$ & \\
\hline VI- 6 & $\begin{array}{l}\text { Tractor impacts fuel tank } \\
\text { during prep for departure }\end{array}$ & $\begin{array}{l}\text { Vehicle } \\
\text { impact }\end{array}$ & $\begin{array}{l}\text { Fire } \\
\text { overpressure }\end{array}$ & $\begin{array}{l}\text { Vehicle fuel fire and tire fire impacts STS and } \\
\text { facility. Programmatic impact and potential } \\
\text { impact to STS if only type A qualified. } \\
\text { Uncontrolled release of sludge, worker and } \\
\text { public dose. }\end{array}$ & $\begin{array}{l}\text { Sloped access pad that directs leaking fuel away } \\
\text { from building. }\end{array}$ & & $\begin{array}{l}\text { STS analysis/qualification to survive the } \\
\text { crash and fire would provide acceptable } \\
\text { containment in this scenario. Not clear } \\
\text { whether that analysis will be retained } \\
\text { under the SPA. }\end{array}$ \\
\hline VI-7 & $\begin{array}{l}\text { Vehicle impacts bottle } \\
\text { storage }\end{array}$ & $\begin{array}{l}\text { Vehicle } \\
\text { Impact }\end{array}$ & deflagration & $\begin{array}{l}\text { Uncontrolled release Loss of inerting } \\
\text { capability, potential bottle missile damage to } \\
\text { ventilation capability and hydrogen buildup to } \\
\text { potential Uncontrolled release of sludge, } \\
\text { worker and public dose. }\end{array}$ & $\begin{array}{l}\text { Vehicle barriers around structure, especially } \\
\text { inerting equipment; } \\
\text { Robust structure around HVAC equipment }\end{array}$ & None. & \\
\hline VI-8 & \begin{tabular}{|l|}
$\begin{array}{l}\text { Vehicle impacts } \\
\text { personnel }\end{array}$ \\
\end{tabular} & \begin{tabular}{|l|} 
Vehicle \\
Impact \\
\end{tabular} & Industrial hazard & Personnel could be injured by vehicle impact & None & None & \\
\hline \multicolumn{8}{|c|}{ AIR CRAFT CRASH } \\
\hline ACC-1 & $\begin{array}{l}\text { Aircraft impacts Modified } \\
\text { Annex }\end{array}$ & $\begin{array}{l}\text { Air Craft } \\
\text { Crash }\end{array}$ & \begin{tabular}{|l|} 
Spray leak \\
Splash and splatter, \\
spill, \\
deflagration, \\
overpressure \\
\end{tabular} & $\begin{array}{l}\text { Potential source of uncontrolled release of } \\
\text { sludge, worker and public dose. Is expected to } \\
\text { be BEU. }\end{array}$ & N/A & $\mathrm{N} / \mathrm{A}$ & $\begin{array}{l}\text { Aircraft crash is beyond extremely } \\
\text { unlikely (need to provide updated analysis } \\
\text { for annex profile). }\end{array}$ \\
\hline \multicolumn{8}{|c|}{ EXTERNAL EXPLOSION } \\
\hline EE-1 & $\begin{array}{l}\text { Damage to Modified } \\
\text { Annex during departure } \\
\text { prep }\end{array}$ & \begin{tabular}{|l|} 
External \\
Explosion \\
(see remarks)
\end{tabular} & overpressure. & $\begin{array}{l}\text { Damage prevents STS departure allowing gas } \\
\text { accumulation and Uncontrolled release of } \\
\text { sludge, worker and public dose. }\end{array}$ & $\begin{array}{l}\text { Inert gas purge capability, } \\
\text { STS vent and purge ports, port tools }\end{array}$ & $\begin{array}{l}\text { Administrative } \\
\text { requirement to monitor } \\
\text { pressure and vent STS } \\
\text { when delayed. }\end{array}$ & $\begin{array}{l}\text { External explosion involves fueling } \\
\text { vehicle or propane utility vehicles }\end{array}$ \\
\hline EE-2 & $\begin{array}{l}\text { Initiates Modified Annex } \\
\text { Facility Fire }\end{array}$ & \begin{tabular}{|l|} 
External \\
Explosion
\end{tabular} & $\begin{array}{l}\text { External Explosion leads to } \\
\text { facility fire and attendant } \\
\text { hazardous conditions }\end{array}$ & $\begin{array}{l}\text { See Facility Fire FF-1 through } 5 \text { for hazardous } \\
\text { conditions, consequences, and candidate } \\
\text { controls }\end{array}$ & $\begin{array}{l}\text { Non combustible materials of construction (2 hour } \\
\text { barrier) }\end{array}$ & $\begin{array}{l}\text { Administrative traffic } \\
\text { restrictions. }\end{array}$ & \\
\hline
\end{tabular}




\begin{tabular}{|c|c|c|c|c|c|c|c|}
\hline \multicolumn{8}{|c|}{ TABLE C-1 "WHAT/IF" HAZARD ANALYSIS STUDY RESULTS - NPH AND EXTERNAL EVENTS } \\
\hline Identifier & "What/If" & Causes & Hazardous Condition & Consequences & $\begin{array}{l}\text { Candidate Controls } \\
\text { Engineered Features }\end{array}$ & Admin Controls & Remarks/Actions \\
\hline EE-3 & Initiates LOEP & $\begin{array}{l}\text { External } \\
\text { Explosion }\end{array}$ & $\begin{array}{l}\text { External Explosion leads to } \\
\text { LOEP and attendant } \\
\text { hazardous conditions } \mathrm{s}\end{array}$ & $\begin{array}{l}\text { See LOEP-1 through } 5 \text { for hazardous } \\
\text { conditions, consequences and candidate } \\
\text { controls }\end{array}$ & N/A & N/A & \\
\hline EE-4 & $\begin{array}{l}\text { Damage to Modified } \\
\text { Annex structure, } \\
\text { impacting transfer lines in } \\
\text { service }\end{array}$ & $\begin{array}{l}\text { External } \\
\text { Explosion }\end{array}$ & $\begin{array}{l}\text { Spray leak } \\
\text { Splash and splatter }\end{array}$ & $\begin{array}{l}\text { Blocked or failed transfer lines result in } \\
\text { Uncontrolled release of sludge, worker and } \\
\text { public dose. }\end{array}$ & Robust structure design resists external explosion & $\begin{array}{l}\text { Administrative traffic } \\
\text { restrictions. }\end{array}$ & \\
\hline EE-5 & $\begin{array}{l}\text { Damage to Modified } \\
\text { Annex structure }\end{array}$ & $\begin{array}{l}\text { External } \\
\text { Explosion }\end{array}$ & Industrial hazard & $\begin{array}{l}\text { Personnel could be injured by structural } \\
\text { failure }\end{array}$ & None & None & \\
\hline \multicolumn{8}{|c|}{ EXTERNAL FACILITY FIRE } \\
\hline EFF-1 & $\begin{array}{l}\text { Initiates Modified Annex } \\
\text { Facility Fire }\end{array}$ & \begin{tabular}{|l} 
External \\
Facility Fire
\end{tabular} & $\begin{array}{l}\text { External facility fire leads to } \\
\text { facility fire and attendant } \\
\text { hazardous conditions }\end{array}$ & $\begin{array}{l}\text { See facility fire for hazardous conditions and } \\
\text { consequences }\end{array}$ & 2 hour and 3 hour barriers, fire suppression system & $\begin{array}{l}\text { Combustible loading } \\
\text { restrictions }\end{array}$ & $\begin{array}{l}\text { Existing KW Basin Annex is the assumed } \\
\text { location of the external facility fire }\end{array}$ \\
\hline EFF-2 & $\begin{array}{l}\text { Initiates KW Basin super- } \\
\text { structure collapse against } \\
\text { modified Annex }\end{array}$ & $\begin{array}{l}\text { External } \\
\text { Facility Fire }\end{array}$ & $\begin{array}{l}\text { deflagration } \\
\text { overpressure. }\end{array}$ & $\begin{array}{l}\text { Impacted structure prevents ventilation of } \\
\text { STSC or departure of loaded STS, hydrogen } \\
\text { accumulation and Uncontrolled release of } \\
\text { sludge, worker and public dose. }\end{array}$ & $\begin{array}{l}3 \text { hour barrier between structures, } \\
\text { Structure separation from existing annex, }\end{array}$ & None & \\
\hline EFF-3 & $\begin{array}{l}\text { Initiates KW Basin super- } \\
\text { structure collapse against } \\
\text { modified Annex }\end{array}$ & $\begin{array}{l}\text { External } \\
\text { Facility Fire }\end{array}$ & Industrial hazard & $\begin{array}{l}\text { Personnel could be injured by structural } \\
\text { collapse }\end{array}$ & None & None & \\
\hline EFF-4 & Initiates LOEP & $\begin{array}{l}\text { External } \\
\text { Facility Fire }\end{array}$ & $\begin{array}{l}\text { LOEP Related hazardous } \\
\text { conditions listed above. }\end{array}$ & LOEP Related consequences listed above. & N/A & $\mathrm{N} / \mathrm{A}$ & \\
\hline EFF-5 & Smoke plugs ventilation & $\begin{array}{l}\text { External } \\
\text { Facility Fire }\end{array}$ & deflagration & $\begin{array}{l}\text { Smoke and soot plugs HVAC, preventing } \\
\text { active ventilation of the STSC, buildup of } \\
\text { hydrogen and Uncontrolled release of sludge, } \\
\text { worker and public dose. }\end{array}$ & $\begin{array}{l}\text { HVAC design that accommodates or mitigates } \\
\text { range fire smoke, } \\
\text { STSC inerting system that functions during and } \\
\text { after range fire }\end{array}$ & $\begin{array}{l}\text { Administrative control } \\
\text { to purge STSC if } \\
\text { ventilation is lost }\end{array}$ & \\
\hline \multicolumn{8}{|c|}{ FACILITY FIRE } \\
\hline $\mathrm{FF}-1$ & $\begin{array}{l}\text { Fire leads to transfer hose } \\
\text { damage }\end{array}$ & Facility fire & $\begin{array}{l}\text { Splash and Splatter } \\
\text { Spray leak }\end{array}$ & $\begin{array}{l}\text { Fire damages transfer, decant or overfill } \\
\text { recovery transfer line (either directly or via } \\
\text { structure failure) resulting in Uncontrolled } \\
\text { release of sludge, worker and public dose. }\end{array}$ & $\begin{array}{l}\text { Facility materials of construction, } \\
\text { Shielded enclosure for transfer hoses, } \\
\text { Fire suppression system }\end{array}$ & $\begin{array}{l}\text { Limited combustible } \\
\text { loading } \\
\text { Control ignition sources }\end{array}$ & \\
\hline $\mathrm{FF}-2$ & $\begin{array}{l}\text { Fire causes loss of } \\
\text { Modified Annex } \\
\text { ventilation fans or } \\
\text { plugging filters }\end{array}$ & Facility Fire & deflagration. & $\begin{array}{l}\text { Loss of STSC active ventilation allows } \\
\text { hydrogen accumulation and Uncontrolled } \\
\text { release of sludge, worker and public dose. }\end{array}$ & Inert gas purge capability, & $\begin{array}{l}\text { Limited combustible } \\
\text { loading } \\
\text { Control ignition sources } \\
\text { Administrative control } \\
\text { to purge STSC if } \\
\text { ventilation is lost. }\end{array}$ & Assumes post fire access \\
\hline
\end{tabular}




\begin{tabular}{|c|c|c|c|c|c|c|c|}
\hline \multicolumn{8}{|c|}{ TABLE C-1 “WHAT/IF” HAZARD ANALYSIS STUDY RESULTS - NPH AND EXTERNAL EVENTS } \\
\hline Identifier & "What/If" & Causes & Hazardous Condition & Consequences & $\begin{array}{c}\text { Candidate Controls } \\
\text { Engineered Features }\end{array}$ & Admin Controls & Remarks/Actions \\
\hline FF-3 & $\begin{array}{l}\text { Modified Annex Facility } \\
\text { fire impacts loaded STSC } \\
\text { or STS }\end{array}$ & Facility Fire & $\begin{array}{c}\text { Overpressure } \\
\text { Deflagration }\end{array}$ & $\begin{array}{l}\text { Fire heats STSC, fails hoses, or heats loaded } \\
\text { STS. Leads to loss of active ventilation for } \\
\text { STSC/deflagration and potentially an } \\
\text { overpressure for the STS if loaded. } \\
\text { Uncontrolled release of sludge, worker and } \\
\text { public dose. }\end{array}$ & $\begin{array}{l}\text { Material of construction limit combustibles, } \\
\text { STS trailer resistance to fire, } \\
\text { Fire suppression system, } \\
\text { Inert gas purge system; } \\
\text { STS vent and purge ports, port tools }\end{array}$ & $\begin{array}{l}\text { Limited combustible } \\
\text { loading, } \\
\text { Control ignition sources } \\
\text { Administrative control } \\
\text { to purge STSC if } \\
\text { ventilation is lost; } \\
\text { Administrative } \\
\text { requirement to monitor } \\
\text { pressure and vent STS } \\
\text { when delayed }\end{array}$ & $\begin{array}{l}\text { Flammable conditions in STSC and } \\
\text { overpressure in STS occur after substantial } \\
\text { time based on CSDR analysis allowing fire } \\
\text { suppression followed by hydrogen/gas } \\
\text { vent and purge. }\end{array}$ \\
\hline FF-4 & $\begin{array}{l}\text { Modified Annex Facility } \\
\text { fire impacts structure }\end{array}$ & \begin{tabular}{|l} 
Facility Fire \\
\end{tabular} & Overturn STSC, Spill & $\begin{array}{l}\text { Structure failure leads to overturn of loaded } \\
\text { STSC and Uncontrolled release of sludge, } \\
\text { worker and public dose. }\end{array}$ & $\begin{array}{l}\text { Material of construction and fire resistance of } \\
\text { structure; } \\
\text { Fire suppression system }\end{array}$ & $\begin{array}{l}\text { Limited combustible } \\
\text { loading, } \\
\text { Control ignition sources }\end{array}$ & \\
\hline FF-5 & $\begin{array}{l}\text { Modified Annex Facility } \\
\text { fire impacts structure }\end{array}$ & Facility Fire & Industrial hazard & $\begin{array}{l}\text { Personnel could be injured by structural } \\
\text { failures }\end{array}$ & $\begin{array}{l}\text { Material of construction and fire resistance of } \\
\text { structure; } \\
\text { Fire suppression system }\end{array}$ & $\begin{array}{l}\text { Limited combustible } \\
\text { loading, } \\
\text { Control ignition sources }\end{array}$ & \\
\hline FF-6 & $\begin{array}{l}\text { Loaded STS not able to } \\
\text { leave }\end{array}$ & Facility Fire & \begin{tabular}{|l} 
overpressure. \\
.
\end{tabular} & $\begin{array}{l}\text { Fire damage prevents transfer of loaded STS, } \\
\text { resulting in gas accumulation and potential } \\
\text { Uncontrolled release of sludge, worker and } \\
\text { public dose. }\end{array}$ & $\begin{array}{l}\text { Material of construction and fire resistance of } \\
\text { structure; } \\
\text { Fire suppression system } \\
\text { STS vent and purge ports, port tools; } \\
\text { Inert gas purge capability }\end{array}$ & $\begin{array}{l}\text { Limited combustible } \\
\text { loading, } \\
\text { Control ignition sources } \\
\text { Administrative } \\
\text { requirement to monitor } \\
\text { pressure and vent STS } \\
\text { when delayed. }\end{array}$ & $\begin{array}{l}\text { Flammable conditions in STSC and } \\
\text { overpressure in STS occur after substantial } \\
\text { time allowing fire suppression followed by } \\
\text { hydrogen/gas vent and purge. }\end{array}$ \\
\hline FF-7 & $\begin{array}{l}\text { Mechanical room fire } \\
\text { causes loss of pneumatics } \\
\text { (LOP) }\end{array}$ & Facility Fire & $\begin{array}{l}\text { Loss of pneumatics from } \\
\text { various operating conditions } \\
\text { to be considered as part of } \\
\text { FMEA }\end{array}$ & $\mathrm{N} / \mathrm{A}$ & $\mathrm{N} / \mathrm{A}$ & N/A & \\
\hline
\end{tabular}


PRC-STP-00473 REV 0

APPENDIX D

"WHAT/IF" HAZARD ANALYSIS RESULTS

OPERATION SEQUENCES (GAP ANALYSIS)

D-i 
Table D-1 "What/If" HAZARDS ANALYSIS RESULTS- OPERATION SEQUENCES (GAP ANALYSIS)

\begin{tabular}{|c|c|c|c|c|c|c|c|}
\hline \multirow[t]{2}{*}{ Identifier* } & \multirow[t]{2}{*}{ "What/If" } & \multirow[t]{2}{*}{ Causes } & \multirow{2}{*}{$\begin{array}{l}\text { Hazardous } \\
\text { Condition }\end{array}$} & \multirow[t]{2}{*}{ Consequences } & \multicolumn{2}{|c|}{ Candidate Controls } & \multirow[t]{2}{*}{ Remarks/Actions } \\
\hline & & & & & Engineered Features & Admin Controls & \\
\hline \multicolumn{8}{|c|}{ Mode 1 PREPARE STSC } \\
\hline $1-2$ & \multicolumn{7}{|c|}{ Position and align STS trailer on weigh scales } \\
\hline $1-2.1$ & $\begin{array}{l}\text { Tractor exhaust } \\
\text { in building }\end{array}$ & $\begin{array}{l}\text { Tractor exhaust location, not } \\
\text { connected to hose to route } \\
\text { outside }\end{array}$ & industrial hazard & $\begin{array}{l}\text { Exhaust is asphyxiant in high concentration, leading to } \\
\text { personnel injury or death }\end{array}$ & $\mathrm{N} / \mathrm{A}$ & N/A & \\
\hline $1-2.2$ & $\begin{array}{l}\text { Back over person } \\
\text { due to lack of } \\
\text { visibility }\end{array}$ & $\begin{array}{l}\text { Error during placement of } \\
\text { tractor }\end{array}$ & industrial hazard & Moving vehicle impact results in personnel injury or death & N/A & N/A & \\
\hline $1-3$ & \multicolumn{7}{|l|}{ Secure STS trailer } \\
\hline $1-3.1$ & $\begin{array}{l}\text { Trailer not } \\
\text { secured }\end{array}$ & Steps not taken & industrial hazard & Trailer moves, potential impact to personnel or equipment & N/A & $\mathrm{N} / \mathrm{A}$ & Step not performed \\
\hline $1-3.2$ & $\begin{array}{l}\text { Trailer secured } \\
\text { improperly }\end{array}$ & Steps not taken & None & $\begin{array}{l}\text { Operational upset. Weight bias or erroneous results lead to } \\
\text { wrong shipping weight. }\end{array}$ & N/A & N/A & $\begin{array}{l}\text { Not a tare or loaded weight. Reference } \\
\text { weights will be well known and easy to } \\
\text { check. }\end{array}$ \\
\hline $1-4$ & \multicolumn{7}{|c|}{ Disconnect STS tractor and exit loading bay ramp } \\
\hline $1-4.1$ & $\begin{array}{l}\text { Driver falls off } \\
\text { tractor }\end{array}$ & Lack of railing & industrial hazard & Inadequate fall protection, personnel injury & N/A & N/A & \\
\hline $1-4.2$ & $\begin{array}{l}\text { Tractor will not } \\
\text { start }\end{array}$ & Mechanical failure & None & $\begin{array}{l}\text { Operational upset. Door cannot close for confinement, fueled } \\
\text { equipment cannot leave, cannot start processing. }\end{array}$ & N/A & N/A & \\
\hline $1-5$ & \multicolumn{7}{|c|}{ Close Annex loading bay door } \\
\hline $1-5.1$ & $\begin{array}{l}\text { Door doesn't } \\
\text { close }\end{array}$ & Mechanical failure & None & $\begin{array}{l}\text { Operational upset. Lack of confinement, cannot proceed to } \\
\text { load, confinement not established }\end{array}$ & N/A & N/A & \\
\hline $1-6$ & \multicolumn{7}{|c|}{ Record trailer weight (WIT-740-401) and verify shipping manifest. } \\
\hline $1-6.1$ & $\begin{array}{l}\text { Trailer mis- } \\
\text { weighed }\end{array}$ & trailer mis-positioned & None & Operational upset. Inadequate monitoring of load weight. & N/A & N/A & $\begin{array}{l}\text { Reference weights will be well known } \\
\text { and easy to check. This is not the key tare } \\
\text { value. }\end{array}$ \\
\hline $1-7$ & \multicolumn{7}{|c|}{ Remove IP-2 bag from Drain Port } \\
\hline $1-7.1$ & $\begin{array}{l}\text { Zipper stuck on } \\
\text { outer IP-2 }\end{array}$ & Faulty bag & None & Operational upset. Process delay, repair bag & N/A & N/A & \\
\hline
\end{tabular}


Table D-1 "What/If" HAZARDS ANALYSIS RESULTS- OPERATION SEQUENCES (GAP ANALYSIS)

\begin{tabular}{|c|c|c|c|c|c|c|c|}
\hline \multirow[t]{2}{*}{ Identifier* } & \multirow[t]{2}{*}{ "What/If" } & \multirow[t]{2}{*}{ Causes } & \multirow{2}{*}{$\begin{array}{c}\text { Hazardous } \\
\text { Condition }\end{array}$} & \multirow[t]{2}{*}{ Consequences } & \multicolumn{2}{|c|}{ Candidate Controls } & \multirow[t]{2}{*}{ Remarks/Actions } \\
\hline & & & & & Engineered Features & Admin Controls & \\
\hline $1-7.2$ & \begin{tabular}{|l} 
Rip or damage \\
IP-2
\end{tabular} & Error, operational event & None & Operational upset. Process delay, repair bag & N/A & N/A & \\
\hline $1-8$ & \multicolumn{7}{|c|}{ Remove Cask drain port cap and install drain port tool } \\
\hline $1-8.1$ & $\begin{array}{l}\text { Contamination } \\
\text { under cap }\end{array}$ & Previously contaminated & $\begin{array}{l}\text { Spread of } \\
\text { contamination, }\end{array}$ & Operational upset, RadCon event & N/A & N/A & \\
\hline $1-8.2$ & Tool won't seat & Threads fouled & None & Operational upset, Repair & N/A & N/A & \\
\hline $1-9$ & \multicolumn{6}{|c|}{ Attach Purge Line H-602 to drain port tool. Align V-609 to "Cask" position. Verify V-607 and V-608 are closed. } & \\
\hline $1-9.1$ & $\begin{array}{l}\text { Purge line won’t } \\
\text { connect }\end{array}$ & Connector failure & None & Operational upset, Repair & $\mathrm{N} / \mathrm{A}$ & $\mathrm{N} / \mathrm{A}$ & \\
\hline $1-9.2$ & $\begin{array}{l}\text { Purge line } \\
\text { connected, but } \\
\text { leaking }\end{array}$ & Connector failure, error & None & Operational upset, Repair & N/A & & \\
\hline $1-9.3$ & \begin{tabular}{|l|} 
Valves not \\
properly \\
positioned closed
\end{tabular} & Error & None & Inerting gas to work area, personnel hazard at Step 1-11 & N/A & $\mathrm{N} / \mathrm{A}$ & \\
\hline 1.10 & \multicolumn{6}{|c|}{ Attach drain line $\mathrm{H}-603$ to drain port tool } & \\
\hline 1.10 .1 & $\begin{array}{l}\text { Purge line won't } \\
\text { connect }\end{array}$ & Connector failure & None & Operational upset, Repair & N/A & N/A & \\
\hline $1-10.2$ & \begin{tabular}{|l} 
Purge line \\
connected, but \\
leaking \\
\end{tabular} & Connector failure, error & None & Operational upset, Repair & N/A & N/A & \\
\hline $1-11$ & \multicolumn{6}{|l|}{ Open drain port } & \\
\hline $1-11.1$ & $\begin{array}{l}\text { Drain port won't } \\
\text { open }\end{array}$ & Frozen/locked threads & None & Operational upset, Repair & N/A & N/A & \\
\hline $1-11.2$ & \begin{tabular}{|l|} 
Gas exits without \\
being routed to \\
drain tank
\end{tabular} & Pressure vents to operating area & $\begin{array}{l}\text { spread of } \\
\text { contamination }\end{array}$ & Operational upset, RadCon event & N/A & N/A & \\
\hline $1-12$ & \multicolumn{7}{|c|}{ Verify STS cask pressure is 0 psig (PI-760-606) } \\
\hline $1-12-1$ & $\begin{array}{l}\text { Pressure indicator } \\
\text { doesn't read zero }\end{array}$ & HVAC pressure less than zero & None & Operational upset & N/A & N/A & \\
\hline
\end{tabular}


Table D-1 "What/If" HAZARDS ANALYSIS RESULTS- OPERATION SEQUENCES (GAP ANALYSIS)

\begin{tabular}{|c|c|c|c|c|c|c|c|}
\hline \multirow[t]{2}{*}{ Identifier* } & \multirow[t]{2}{*}{ "What/If" } & \multirow[t]{2}{*}{ Causes } & \multirow{2}{*}{$\begin{array}{l}\text { Hazardous } \\
\text { Condition }\end{array}$} & \multirow[t]{2}{*}{ Consequences } & \multicolumn{2}{|c|}{ Candidate Controls } & \multirow[t]{2}{*}{ Remarks/Actions } \\
\hline & & & & & Engineered Features & Admin Controls & \\
\hline $1-13$ & \multicolumn{7}{|c|}{ Unzip/remove Outer IP-2 bag. } \\
\hline $1-13.1$ & \begin{tabular}{|l|}
$\begin{array}{l}\text { Operator falls off } \\
\text { platform on } \\
\text { trailer }\end{array}$ \\
\end{tabular} & $\begin{array}{l}\text { Fall protection not provided on } \\
\text { trailer }\end{array}$ & Industrial hazard & \begin{tabular}{|l|} 
Personnel injury \\
\end{tabular} & N/A & $\mathrm{N} / \mathrm{A}$ & \\
\hline $1-13.2$ & $\begin{array}{l}\text { Zipper stuck on } \\
\text { outer IP-2 }\end{array}$ & Faulty bag & None & Operational upset. Process delay, repair bag & N/A & N/A & \\
\hline $1-13.3$ & $\begin{array}{l}\text { Rip or damage } \\
\text { IP-2 }\end{array}$ & Error, operational event & None & Operational upset. Process delay, repair bag & N/A & $\mathrm{N} / \mathrm{A}$ & Replace in this operating window \\
\hline $1-14$ & \multicolumn{6}{|c|}{ Unzip/remove Inner IP-2 bag. } & \\
\hline $1-14.1$ & \begin{tabular}{|l|} 
Operator falls off \\
platform on \\
trailer
\end{tabular} & $\begin{array}{l}\text { Fall protection not provided on } \\
\text { trailer }\end{array}$ & Industrial hazard & Personnel injury & $\mathrm{N} / \mathrm{A}$ & N/A & \\
\hline $1-14.2$ & $\begin{array}{l}\text { Zipper stuck on } \\
\text { outer IP-2 }\end{array}$ & Faulty bag & None & Operational upset. Process delay, repair bag & $\mathrm{N} / \mathrm{A}$ & N/A & \\
\hline $1-14.3$ & $\begin{array}{l}\text { Rip or damage } \\
\text { IP-2 }\end{array}$ & Error, operational event & None & Operational upset. Process delay, repair bag & N/A & N/A & \\
\hline $1-14.4$ & \begin{tabular}{|l|} 
Outside cask \\
contaminated \\
under IP-2 \\
\end{tabular} & Previously contaminated & $\begin{array}{l}\text { Spread of } \\
\text { contamination }\end{array}$ & Operational upset, RadCon event & $\mathrm{N} / \mathrm{A}$ & N/A & Stop process and decon \\
\hline $1-15$ & \multicolumn{7}{|c|}{ Install Lid Lift Fixture on STS cask lid } \\
\hline $1-15.1$ & \begin{tabular}{|l|} 
Lift fixture not \\
properly attached
\end{tabular} & Rigging error & Industrial hazard & $\begin{array}{l}\text { Operational upset. Drop of load, Damage to cask, STSC, } \\
\text { personnel }\end{array}$ & $\mathrm{N} / \mathrm{A}$ & N/A & \\
\hline $1-16$ & \multicolumn{7}{|c|}{ Remove the STS Lid bolts } \\
\hline $1-16.1$ & \begin{tabular}{|l|l|}
$\begin{array}{l}\text { A bolt not } \\
\text { removed }\end{array}$ \\
\end{tabular} & Error & None & Operational upset, Lid not free to remove & $\mathrm{N} / \mathrm{A}$ & N/A & $\begin{array}{l}\text { Lift with bolt on leads to cask or lid } \\
\text { damage, see } 1-17.2\end{array}$ \\
\hline $1-17$ & \multicolumn{7}{|c|}{$\begin{array}{l}\text { Remove the STS Lid and install onto the Lid Inspection } \\
\text { Station. }\end{array}$} \\
\hline $1-17.1$ & $\begin{array}{l}\text { Under side of lid } \\
\text { contaminated }\end{array}$ & Previously contaminated & $\begin{array}{l}\text { Spread of } \\
\text { contamination }\end{array}$ & Operational upset, RadCon event & N/A & N/A & Add step to paper the inspection station \\
\hline $1-17.2$ & $\begin{array}{l}\text { Lid stuck, crane } \\
\text { overload or shear } \\
\text { bolt }\end{array}$ & Bolts not all removed & Industrial hazard & Operational upset, Damage to cask or crane, personnel injury & N/A & N/A & Possible? \\
\hline
\end{tabular}


Table D-1 "What/If" HAZARDS ANALYSIS RESULTS- OPERATION SEQUENCES (GAP ANALYSIS)

\begin{tabular}{|c|c|c|c|c|c|c|c|}
\hline \multirow[t]{2}{*}{ Identifier* } & \multirow[t]{2}{*}{ "What/If" } & \multirow[t]{2}{*}{ Causes } & \multirow{2}{*}{$\begin{array}{l}\text { Hazardous } \\
\text { Condition }\end{array}$} & \multirow[t]{2}{*}{ Consequences } & \multicolumn{2}{|c|}{ Candidate Controls } & \multirow[t]{2}{*}{ Remarks/Actions } \\
\hline & & & & & Engineered Features & Admin Controls & \\
\hline $1-17.3$ & $\begin{array}{l}\text { Access to lid } \\
\text { inspection station } \\
\text { leads to fall. }\end{array}$ & No fall protection & Industrial hazard & Personnel injury & $\mathrm{N} / \mathrm{A}$ & N/A & \\
\hline $1-17.4$ & $\begin{array}{l}\text { Wrong STSC } \\
\text { type (annulus } \\
\text { versus not) }\end{array}$ & Error at T Plant & None & $\begin{array}{l}\text { Cask overpressure Potential to load material in wrong STSC } \\
\text { type with future thermal transient, cask overpressure if not } \\
\text { detected, or return to T Plant to get proper STSC. }\end{array}$ & $\mathrm{N} / \mathrm{A}$ & N/A & $\begin{array}{l}\text { Differences in loaded weights and Tares } \\
\text { make detection of error fairly certain. }\end{array}$ \\
\hline $1-18$ & \multicolumn{7}{|c|}{ Verify V-103, V-109 are OPEN. Verify V-101, AOV-104 and AOV-105 are CLOSED } \\
\hline $1-18.1$ & $\begin{array}{l}\text { Valves not } \\
\text { properly } \\
\text { positioned open }\end{array}$ & Error & None & $\begin{array}{l}\text { Operational upset, Line not vented to transfer box, Potential } \\
\text { spread of contamination at step 1-20 }\end{array}$ & $\mathrm{N} / \mathrm{A}$ & N/A & \\
\hline $1-18.2$ & $\begin{array}{l}\text { Valve V-101 not } \\
\text { properly } \\
\text { positioned closed }\end{array}$ & Error & None & $\begin{array}{l}\text { Operational upset, RadCon event Transfer line to basin open to } \\
\text { transfer box, Some potential to spread contamination into } \\
\text { valve box, leak detection at future step }\end{array}$ & $\mathrm{N} / \mathrm{A}$ & N/A & \\
\hline $1-18.3$ & $\begin{array}{l}\text { Valve AOV-104 } \\
\text { not properly } \\
\text { positioned closed }\end{array}$ & Error & None & Operational upset premature filling of STSC with IXM water & $\mathrm{N} / \mathrm{A}$ & N/A & \\
\hline $1-19$ & \multicolumn{7}{|c|}{ Remove and store the 3-bolt blind flange from STSC Nozzle A. } \\
\hline $1-19.1$ & $\begin{array}{l}\text { Outside of } \\
\text { STSC/flange } \\
\text { contaminated }\end{array}$ & Previously contaminated flange & $\begin{array}{l}\text { Spread of } \\
\text { contamination }\end{array}$ & Operational upset, RadCon event & $\mathrm{N} / \mathrm{A}$ & $\mathrm{N} / \mathrm{A}$ & \\
\hline 1.20 & \multicolumn{7}{|c|}{ Remove contamination controls and end cap from Sludge line connector. Air flows from the room into the TLSB through H- 107 and H-107 annulus. IL-125 turns ON. } \\
\hline $1-20.1$ & $\begin{array}{l}\text { Contaminated } \\
\text { liquid in line } \\
\text { connector }\end{array}$ & $\begin{array}{l}\text { Thermal expansion or } \\
\text { inadequate drying }\end{array}$ & $\begin{array}{l}\text { spread of } \\
\text { contamination }\end{array}$ & $\begin{array}{l}\text { Operational upset, RadCon event Spill of contaminated liquid } \\
\text { in work area, onto STSC, or into STS cask }\end{array}$ & $\mathrm{N} / \mathrm{A}$ & N/A & \\
\hline $1-20.2$ & $\begin{array}{l}\text { No noticeable } \\
\text { vacuum }\end{array}$ & $\begin{array}{l}\text { Valve alignment error at step 1- } \\
19\end{array}$ & $\begin{array}{l}\text { Spread of } \\
\text { contamination }\end{array}$ & $\begin{array}{l}\text { Operational upset, RadCon event } \\
\text { Dry material in hose not contained by air flow leading to local } \\
\text { contamination spread }\end{array}$ & $\mathrm{N} / \mathrm{A}$ & N/A & \\
\hline $1-20.3$ & $\begin{array}{l}\text { Light doesn't } \\
\text { light }\end{array}$ & $\begin{array}{l}\text { Light burned out, connection } \\
\text { not made. }\end{array}$ & None & Operational upset, repair & $\mathrm{N} / \mathrm{A}$ & N/A & \\
\hline $1-20.4$ & $\begin{array}{l}\text { Light doesn't } \\
\text { light }\end{array}$ & Flow instrument failure & None & Operational upset, repair & $\mathrm{N} / \mathrm{A}$ & $\mathrm{N} / \mathrm{A}$ & \\
\hline $1-21$ & \multicolumn{7}{|c|}{ Connect the Sludge Inlet Riverbend connector (Hose H-107) to the 3 bolt PUP connector on Nozzle A and torque the acorn nuts. Verify IL-118 is ON. } \\
\hline 1.21 .1 & $\begin{array}{l}\text { Light doesn't } \\
\text { light }\end{array}$ & $\begin{array}{l}\text { Light burned out, connection } \\
\text { not made }\end{array}$ & None & Operational upset, interlock not cleared, Rework & $\mathrm{N} / \mathrm{A}$ & N/A & \\
\hline
\end{tabular}


Table D-1 "What/If" HAZARDS ANALYSIS RESULTS- OPERATION SEQUENCES (GAP ANALYSIS)

\begin{tabular}{|c|c|c|c|c|c|c|c|}
\hline \multirow[t]{2}{*}{ Identifier* } & \multirow[t]{2}{*}{ "What/If" } & \multirow[t]{2}{*}{ Causes } & \multirow{2}{*}{$\begin{array}{l}\text { Hazardous } \\
\text { Condition }\end{array}$} & \multirow[t]{2}{*}{ Consequences } & \multicolumn{2}{|c|}{ Candidate Controls } & \multirow[t]{2}{*}{ Remarks/Actions } \\
\hline & & & & & Engineered Features & Admin Controls & \\
\hline $1-21.2$ & $\begin{array}{l}\text { Connector will } \\
\text { not seat }\end{array}$ & Faulty connector on STSC & None & Operational upset, interlock not cleared, Repair & N/A & N/A & \\
\hline $1-21.3$ & $\begin{array}{l}\text { Inner line does } \\
\text { not seal }\end{array}$ & $\begin{array}{l}\text { Faulty o-ring or connector at } \\
\text { STSC }\end{array}$ & None & $\begin{array}{l}\text { Operational upset, Radcon event, potential leak at STSC upon } \\
\text { start of pumping at step 2-7 }\end{array}$ & $\begin{array}{l}\text { Connector design and } \\
\text { testing }\end{array}$ & N/A & $\begin{array}{l}\text { Not clear how to detect seal is effective } \\
\text { prior to pumping. Area not occupied } \\
\text { when pumping initiated. Leak detector } \\
\text { would detect leak. A second failure such } \\
\text { as back pressure from within the STSC } \\
\text { (e.g., plugged discharge header) or } \\
\text { overfill would be required to result in a } \\
\text { leak. } \\
\end{array}$ \\
\hline $1-21.4$ & $\begin{array}{l}\text { Acorn nuts not } \\
\text { torqued }\end{array}$ & Error, step skipped & None & $\begin{array}{l}\text { Operational upset, Radcon event, potential leak upon backing } \\
\text { out. Leak at STSC upon start of pumping at step 2-7. }\end{array}$ & $\begin{array}{l}\text { Connector design and } \\
\text { testing }\end{array}$ & N/A & $\begin{array}{l}\text { Not clear how to detect seal is effective } \\
\text { prior to pumping. Area not occupied } \\
\text { when pumping initiated. Leak detector } \\
\text { would detect leak. A second failure such } \\
\text { as back pressure from within the STSC } \\
\text { (e.g., plugged discharge header) or } \\
\text { overfill would be required to result in a } \\
\text { leak. } \\
\end{array}$ \\
\hline $1-22$ & \multicolumn{7}{|c|}{ Verify V-205 and V-201 are OPEN. Verify V-202 and V-206 are CLOSED } \\
\hline $1-22.1$ & $\begin{array}{l}\text { Valves not } \\
\text { opened }\end{array}$ & Error, step skipped & None & $\begin{array}{l}\text { Line not vented to decant box, Potential spread of } \\
\text { contamination at step } 1-25\end{array}$ & N/A & N/A & \\
\hline $1-22.2$ & V-202 not closed & Error, step skipped & None & $\begin{array}{l}\text { Operational upset, RadCon event, ventilation suction of box } \\
\text { connected to decant transfer line and decant pump, potential to } \\
\text { lead to spread of contamination into box, potential liquid spill } \\
\text { to box, leak detection. }\end{array}$ & N/A & N/A & $\begin{array}{l}\text { The intent is to ensure backflow from the } \\
\text { decantbackflush line into the decant box } \\
\text { when removing cover and connecting to } \\
\text { the STSC. If V-202 is open, } \\
\text { contaminated fluids may be sucked into } \\
\text { the decant box. F-201 would likely filter, } \\
\text { but contamination is possible. }\end{array}$ \\
\hline $1-22.3$ & \begin{tabular}{|l|} 
Valve V-206 not \\
properly \\
positioned closed \\
\end{tabular} & Error & None & Operational upset premature filling of STSC with IXM water & N/A & N/A & HVAC valve mis-positioned \\
\hline $1-23$ & \multicolumn{7}{|c|}{ Remove and store the 4-bolt blind flange from STSC Nozzle B. } \\
\hline $1-23.1$ & $\begin{array}{l}\text { Outside of } \\
\text { STSC/flange } \\
\text { contaminated }\end{array}$ & Previously contaminated flange & $\begin{array}{l}\text { Spread of } \\
\text { contamination }\end{array}$ & Operational upset, RadCon event & N/A & $\mathrm{N} / \mathrm{A}$ & \\
\hline $1-24$ & \\
\hline
\end{tabular}


Table D-1 "What/If" HAZARDS ANALYSIS RESULTS- OPERATION SEQUENCES (GAP ANALYSIS)

\begin{tabular}{|c|c|c|c|c|c|c|c|}
\hline \multirow[t]{2}{*}{ Identifier* } & \multirow[t]{2}{*}{ "What/If" } & \multirow[t]{2}{*}{ Causes } & \multirow{2}{*}{$\begin{array}{l}\text { Hazardous } \\
\text { Condition }\end{array}$} & \multirow[t]{2}{*}{ Consequences } & \multicolumn{2}{|c|}{ Candidate Controls } & \multirow[t]{2}{*}{ Remarks/Actions } \\
\hline & & & & & Engineered Features & Admin Controls & \\
\hline $1-24.1$ & $\begin{array}{l}\text { Contaminated } \\
\text { liquid in line } \\
\text { connector }\end{array}$ & $\begin{array}{l}\text { Thermal expansion or } \\
\text { inadequate drying }\end{array}$ & $\begin{array}{l}\text { Spread of } \\
\text { contamination }\end{array}$ & $\begin{array}{l}\text { Operational upset, RadCon event Spill of contaminated liquid } \\
\text { in work area, onto STSC, or into STS cask }\end{array}$ & N/A & N/A & $\begin{array}{l}\text { Thermal expansion of liquid in line from } \\
\text { last draining leads to liquid in hose at } \\
\text { end. Consider adding a valve at P-101 } \\
\text { outlet to drain the slurry transfer line } \\
\text { back to the basin. }\end{array}$ \\
\hline $1-24.2$ & $\begin{array}{l}\text { Light doesn't } \\
\text { light }\end{array}$ & $\begin{array}{l}\text { Light burned out, connection } \\
\text { not made. }\end{array}$ & None & Operational upset, repair & N/A & N/A & \\
\hline $1-24.3$ & $\begin{array}{l}\text { Light doesn't } \\
\text { light }\end{array}$ & Flow instrument failure & None & Operational upset, repair & N/A & N/A & \\
\hline $1-25$ & \multicolumn{7}{|c|}{ Connect the Decant Outlet/Backflush Inlet Riverbend connector (Hose H-203) to the 4 bolt PUP connector on Nozzle B and torque the acorn nuts. Verify IL-218 is ON. } \\
\hline $1-25.1$ & $\begin{array}{l}\text { Light doesn't } \\
\text { light }\end{array}$ & $\begin{array}{l}\text { Light burned out, connection } \\
\text { not made }\end{array}$ & None & Operational upset, interlock not cleared, Rework & N/A & N/A & \\
\hline $1-25.2$ & $\begin{array}{l}\text { Connector will } \\
\text { not seat }\end{array}$ & Faulty connector on STSC & None & Operational upset, interlock not cleared, Repair & N/A & N/A & \\
\hline $1-25.3$ & $\begin{array}{l}\text { Inner line does } \\
\text { not seal }\end{array}$ & $\begin{array}{l}\text { Faulty o-ring or connector at } \\
\text { STSC }\end{array}$ & $\begin{array}{l}\text { Spread of } \\
\text { contamination }\end{array}$ & $\begin{array}{l}\text { Operational upset, Air Leak at STSC upon start of pumping, } \\
\text { ineffective decant at } 4-3 \text { or } 5-2 \text {. } \\
\text { Leak at backflush 6-4, RadCon event }\end{array}$ & N/A & N/A & $\begin{array}{l}\text { Not clear how to detect seal is effective } \\
\text { prior to pumping/ backflush. Leak } \\
\text { detector would detect leak. }\end{array}$ \\
\hline $1-25.4$ & $\begin{array}{l}\text { Acorn nuts not } \\
\text { torqued }\end{array}$ & Error, step skipped & $\begin{array}{l}\text { Spread of } \\
\text { contamination }\end{array}$ & $\begin{array}{l}\text { Operational upset. Leak upon backing out. Air Leak at STSC } \\
\text { upon start of pumping, ineffective decant 4-3 or 5-2. } \\
\text { Leak at backflush 6-4, RadCon event }\end{array}$ & N/A & N/A & $\begin{array}{l}\text { Not clear how to detect seal is effective } \\
\text { prior to pumping/ backflush. Leak } \\
\text { detector would detect leak. }\end{array}$ \\
\hline $1-26$ & \multicolumn{7}{|c|}{ Remove and store the 2 inch Camlok cap from Nozzle F2 and connect the IXM Water Inlet onto the 2 inch Camlok connection (Hose H-752). } \\
\hline $1-26.1$ & $\begin{array}{l}\text { Connector not } \\
\text { properly } \\
\text { connected }\end{array}$ & Error, defective connector & $\begin{array}{l}\text { Spread of } \\
\text { contamination }\end{array}$ & $\begin{array}{l}\text { Operational upset. RadCon event. Leak IXM water when used } \\
\text { at 1-36, water on STSC, in STS }\end{array}$ & N/A & N/A & Low level of contamination \\
\hline $1-27$ & \multicolumn{7}{|c|}{ Remove and store the 4 inch Camlok cap from Nozzle F1 and connect the 4 inch STSC Vent Camlok connection (Hose H- 501). } \\
\hline $1-27.1$ & $\begin{array}{l}\text { Improper } \\
\text { connection }\end{array}$ & Enror, defective connector & N/A & & N/A & N/A & $\begin{array}{l}\text { This would provide a leak path if there } \\
\text { were a way to overfill the STSC up into } \\
\text { the head connectors. }\end{array}$ \\
\hline $1-28$ & \multicolumn{7}{|c|}{ Connect the Purge Inlet 1 inch Quick Connect (Hose H-604). } \\
\hline $1-28.1$ & $\begin{array}{l}\text { Improper, leaky } \\
\text { connection }\end{array}$ & Error, defective connector & Industrial hazard. & $\begin{array}{l}\text { Operational upset; Slow or ineffective inert; may not be } \\
\text { effective if used for backup hydrogen control, potential for } \\
\text { hydrogen buildup and deflagration; inerting gas discharge to } \\
\text { work area }\end{array}$ & Connector design & N/A & $\begin{array}{l}\text { Should hear leak due to low STSC } \\
\text { pressure; if used as backup inerting }\end{array}$ \\
\hline
\end{tabular}


Table D-1 "What/If" HAZARDS ANALYSIS RESULTS- OPERATION SEQUENCES (GAP ANALYSIS)

\begin{tabular}{|c|c|c|c|c|c|c|c|}
\hline \multirow[t]{2}{*}{ Identifier* } & \multirow[t]{2}{*}{ "What/If" } & \multirow[t]{2}{*}{ Causes } & \multirow{2}{*}{$\begin{array}{c}\text { Hazardous } \\
\text { Condition }\end{array}$} & \multirow[t]{2}{*}{ Consequences } & \multicolumn{2}{|c|}{ Candidate Controls } & \multirow[t]{2}{*}{ Remarks/Actions } \\
\hline & & & & & Engineered Features & Admin Controls & \\
\hline $1-28.2$ & $\begin{array}{l}\text { No flow due to } \\
\text { plugged } \\
\text { connector }\end{array}$ & Manufacturing defect & deflagration & $\begin{array}{l}\text { Operational upset; Ineffective inert; not effective if used for } \\
\text { backup hydrogen control, potential for hydrogen buildup and } \\
\text { deflagration at future step }\end{array}$ & $\begin{array}{l}\text { Connector design, purge } \\
\text { flowmeter }\end{array}$ & $\begin{array}{l}\text { Procedures } \\
\text { Training } \\
\text { Conduct of operations }\end{array}$ & Is the connection tested? \\
\hline $1-29$ & \multicolumn{7}{|c|}{ Connect I\&C connector to LDE-710-103 (Leak Detector) and LE-740-401 (Level Detector) Safety Significant instruments } \\
\hline 1-29.1 & $\begin{array}{l}\text { Improper } \\
\text { connection }\end{array}$ & Error & None & $\begin{array}{l}\text { Operational upset, repair/replace Required instruments not } \\
\text { available }\end{array}$ & $\mathrm{N} / \mathrm{A}$ & N/A & $\begin{array}{l}\text { Add in step description to turn on switch } \\
\text { and check connection. Color coded and } \\
\text { keyed to prevent cross connect }\end{array}$ \\
\hline $1-29.2$ & $\begin{array}{l}\text { Detector(s) don't } \\
\text { work }\end{array}$ & $\begin{array}{l}\text { Manufacturing defect, failed } \\
\text { instrument }\end{array}$ & None & $\begin{array}{l}\text { Operational upset, repair/replace Required instruments not } \\
\text { available }\end{array}$ & N/A & N/A & \\
\hline 1.30 & \multicolumn{7}{|c|}{ Connect I\&C connector to LDE-720-204 (Leak Detector) and LSH-740-402 (Level Switch) General Service instruments } \\
\hline $1-30.1$ & $\begin{array}{l}\text { Improper } \\
\text { connection }\end{array}$ & Error & None & Operational upset, repair/replace Instruments not available & N/A & $\mathrm{N} / \mathrm{A}$ & $\begin{array}{l}\text { Add in step description to turn on switch } \\
\text { and check connection. Color coded and } \\
\text { keyed to prevent cross connect. One } \\
\text { switch controls all four instrument } \\
\text { connections. Faulty connection will } \\
\text { cause I-1, I-2, I-3, or LIT Health light to } \\
\text { turn on. }\end{array}$ \\
\hline $1-30.2$ & $\begin{array}{l}\text { Detector(s) don't } \\
\text { work }\end{array}$ & $\begin{array}{l}\text { Manufacturing defect, failed } \\
\text { instrument }\end{array}$ & None & Operational upset, repair/replace Instruments not available & N/A & N/A & \\
\hline $1-31$ & \multicolumn{6}{|c|}{ Turn on STSC Instrument disconnect switch. Verify instrument Health is OK (IL-408 and IL-412 are ON) } & \\
\hline $1-31.1$ & $\begin{array}{l}\text { Fail to turn on } \\
\text { switch }\end{array}$ & Error & None & Operational upset & N/A & N/A & \\
\hline $1-31.2$ & $\begin{array}{l}\text { Light doesn't } \\
\text { light }\end{array}$ & $\begin{array}{l}\text { Light burned out, connection } \\
\text { not made. }\end{array}$ & None & Operational upset, repair & $\mathrm{N} / \mathrm{A}$ & $\mathrm{N} / \mathrm{A}$ & \\
\hline $1-32$ & \multicolumn{7}{|c|}{ Weigh and record initial filling condition weight (WIT-740-401). } \\
\hline $1-32.1$ & $\begin{array}{l}\text { Initial condition } \\
\text { not manually } \\
\text { recorded }\end{array}$ & Error, step skipped & None & Operational upset & $\mathrm{N} / \mathrm{A}$ & N/A & $\begin{array}{l}\text { Data automatically recorded }- \text { need to } \\
\text { verify that recording is functional in steps } \\
1-30,1-31 \text { above }\end{array}$ \\
\hline $1-33$ & \multicolumn{7}{|c|}{ Select Sludge Type on PNL-401 (ECRT-KS-401 to 'K-East', 'KWest', or 'Settler' position) and re } \\
\hline
\end{tabular}


Table D-1 "What/If" HAZARDS ANALYSIS RESULTS- OPERATION SEQUENCES (GAP ANALYSIS)

\begin{tabular}{|c|c|c|c|c|c|c|c|}
\hline \multirow[t]{2}{*}{ Identifier* } & \multirow[t]{2}{*}{ "What/If" } & \multirow[t]{2}{*}{ Causes } & \multirow{2}{*}{$\begin{array}{c}\text { Hazardous } \\
\text { Condition }\end{array}$} & \multirow[t]{2}{*}{ Consequences } & \multicolumn{2}{|c|}{ Candidate Controls } & \multirow[t]{2}{*}{ Remarks/Actions } \\
\hline & & & & & Engineered Features & Admin Controls & \\
\hline $1-33.1$ & Wrong selection & Error & Overpressure & $\begin{array}{l}\text { Wrong calculations made in automatic system, potential to } \\
\text { overload STSC, resulting in lower transport window and lesser } \\
\text { thermal stability. Potential overpressure, worker and public } \\
\text { dose after loading if manual calculation is also in error. }\end{array}$ & & $\begin{array}{l}\text { Reliance on manual } \\
\text { calculation for safety } \\
\text { analysis/TSR } \\
\text { Procedures } \\
\text { Training } \\
\text { Conduct of operations } \\
\end{array}$ & $\begin{array}{l}\text { This anticipates potential overload of } \\
\text { settler sludge based on use of automatec } \\
\text { recording system to determine load, } \\
\text { which may be in error. } \\
\text { May indicate error in manual } \\
\text { recording/calculating } \\
\end{array}$ \\
\hline $1-34$ & \multicolumn{7}{|c|}{ Initiate operability of LSH-740-402: Place ECRT-HS-113 in 'DISABLE' position. } \\
\hline $1-34.1$ & $\begin{array}{l}\text { Fail to put in } \\
\text { DISABLE } \\
\text { position }\end{array}$ & Error & None & $\begin{array}{l}\text { Operational upset. High level interlock will initiate bladder } \\
\text { flush at future step (1-38) or plant condition }\end{array}$ & N/A & N/A & May result in bladder flush attempt \\
\hline $1-35$ & \multicolumn{7}{|c|}{ Record starting value of water meter $\mathrm{FQI}-770-701$} \\
\hline $1-35.1$ & $\begin{array}{l}\text { Wrong number } \\
\text { recorded or not } \\
\text { recorded }\end{array}$ & Error & None & Operational upset, Failure to measure water volume & N/A & N/A & $\begin{array}{l}\text { Does not appear to be automatically } \\
\text { logged }\end{array}$ \\
\hline $1-36$ & \multicolumn{7}{|c|}{ Open Valve STP-V-731 and begin initial fill of STSC with IXM water. } \\
\hline $1-36.1$ & $\begin{array}{l}\text { Water spray/in } \\
\text { cask if } \\
\text { misconnected } \\
\text { flange }\end{array}$ & $\begin{array}{l}\text { Incomplete connection at step } \\
1-27\end{array}$ & $\begin{array}{l}\text { Spread of } \\
\text { contamination }\end{array}$ & $\begin{array}{l}\text { Operational upset, some RadCon consequence due to leaked } \\
\text { IXM water in STSC head and STS cask }\end{array}$ & $\mathrm{N} / \mathrm{A}$ & N/A & Low level of contamination. \\
\hline $1-37$ & \multicolumn{6}{|c|}{ Close V-109 and V-205 } & \\
\hline $1-37.1$ & $\begin{array}{l}\text { Fail to close V- } \\
109\end{array}$ & error & $\begin{array}{l}\text { Leak sludge to } \\
\text { transfer box., } \\
\text { Hydrogen } \\
\text { Deflagration } \\
\end{array}$ & $\begin{array}{l}\text { Operational upset, RadCon upset with sludge in transfer box } \\
\text { Future routing of sludge to transfer box, trip leak detector, } \\
\text { interlock shutdown pump }\end{array}$ & $\begin{array}{l}\text { Secondary confinement } \\
\text { Leak detection }\end{array}$ & \begin{tabular}{|l|} 
Procedures \\
Training \\
Conduct of operations
\end{tabular} & \\
\hline $1-37.2$ & $\begin{array}{l}\text { Fail to close V- } \\
109\end{array}$ & error & $\begin{array}{l}\text { Spray leak } \\
\text { Splash and splatter }\end{array}$ & $\begin{array}{l}\text { Operational upset. Potential for Worker and public dose; } \\
\text { Future routing of sludge to transfer box, trip leak detector, } \\
\text { interlock shutdown pump }\end{array}$ & $\begin{array}{l}\text { Secondary confinement } \\
\text { Leak detection }\end{array}$ & $\begin{array}{l}\text { Procedures } \\
\text { Training } \\
\text { Conduct of operations }\end{array}$ & \\
\hline $1-37.3$ & $\begin{array}{l}\text { Fail to close V- } \\
205\end{array}$ & error & None & Operational upset; air into suction of decant pump & N/A & N/A & \\
\hline $1-38$ & \multicolumn{7}{|c|}{$\begin{array}{l}\text { Level reaches LSH-740-402 level. IL-13 and IL-402 turn ON. Interlock I-3 trips and shuts off IXM water supply valve AOV-701. Bladder flush valve AOV-102 opens. HS-113 'DISABLE' mode prevents bladder flush from occurring) AOV-113 } \\
\text { held closed. }\end{array}$} \\
\hline
\end{tabular}


Table D-1 "What/If" HAZARDS ANALYSIS RESULTS- OPERATION SEQUENCES (GAP ANALYSIS)

\begin{tabular}{|c|c|c|c|c|c|c|c|}
\hline \multirow[t]{2}{*}{ Identifier* } & \multirow[t]{2}{*}{ "What/If" } & \multirow[t]{2}{*}{ Causes } & \multirow{2}{*}{$\begin{array}{l}\text { Hazardous } \\
\text { Condition }\end{array}$} & \multirow[t]{2}{*}{ Consequences } & \multicolumn{2}{|c|}{ Candidate Controls } & \multirow[t]{2}{*}{ Remarks/Actions } \\
\hline & & & & & Engineered Features & Admin Controls & \\
\hline $1-38.1$ & $\begin{array}{l}\text { Level switch does } \\
\text { not function }\end{array}$ & Non functioning instrument & $\begin{array}{l}\text { Spread of } \\
\text { contamination }\end{array}$ & $\begin{array}{l}\text { Operational upset, RadCon event } \\
\text { Overfill STSC, into HVAC, potentially no indication, extensive } \\
\text { water intrusion }\end{array}$ & N/A & N/A & $\begin{array}{l}\text { Need to ensure that level indication is } \\
\text { tracking fill volume incase switch does } \\
\text { not function (manual back up). }\end{array}$ \\
\hline $1-38.2$ & Bladder flush & Test switch does not function & None & $\begin{array}{l}\text { Operational upset, Extra water into STSC, but within head } \\
\text { volume }\end{array}$ & $\mathrm{N} / \mathrm{A}$ & N/A & \\
\hline $1-39$ & \multicolumn{7}{|c|}{ Close Valve STP-V-731 when indicator reflects full STSC. } \\
\hline $1-39.1$ & $\begin{array}{l}\text { Fail to close V- } \\
731\end{array}$ & error & None & $\begin{array}{l}\text { Operational upset, Water flow will restart when decant starts at } \\
\text { step 1-45/1-46 and run until interlock re-asserts }\end{array}$ & N/A & N/A & $\begin{array}{l}\text { AOV-701 reset/open with high interlock } \\
\text { clear. }\end{array}$ \\
\hline $1-40$ & \multicolumn{7}{|c|}{ Record ending value of water meter FQI-770-701 } \\
\hline $1-40.1$ & $\begin{array}{l}\text { Record wrong } \\
\text { value }\end{array}$ & error & None & $\begin{array}{l}\text { Operational upset, Calculate water volume that will differ from } \\
\text { volume calculation for STSC }\end{array}$ & N/A & N/A & \\
\hline $1-41$ & \multicolumn{7}{|c|}{ Drain (V-736) and disconnect the IXM Water (H-752) from Nozzle F2 } \\
\hline $1-41.1$ & $\begin{array}{l}\text { Potential line } \\
\text { water drain }\end{array}$ & Open connector & $\begin{array}{l}\text { Spread of } \\
\text { contamination }\end{array}$ & $\begin{array}{l}\text { Operational upset, RadCon upset } \\
\text { IXM water on STSC and into STS }\end{array}$ & N/A & $\mathrm{N} / \mathrm{A}$ & $\begin{array}{l}\text { No indication of closure on this } \\
\text { connector when in the stored position. }\end{array}$ \\
\hline $1-42$ & \multicolumn{7}{|c|}{ Connect the Purge Outlet 1 inch Quick Connect (Hose H-659) to Nozzle F2. } \\
\hline $1-42.1$ & $\begin{array}{l}\text { Not fully } \\
\text { connected or } \\
\text { action not taken }\end{array}$ & Faulty connector, step omitted & None & $\begin{array}{l}\text { Operational upset, Unable to provide inerting oxygen } \\
\text { monitoring when inerting at end of operation. }\end{array}$ & N/A & $\mathrm{N} / \mathrm{A}$ & $\begin{array}{l}\text { Should have an audible sound from open } \\
\text { or partially open hole. }\end{array}$ \\
\hline $1-43$ & \multicolumn{7}{|c|}{ Verify pressure gauge PI-760-652 is reading at least 1 inch of water vacuum. } \\
\hline $1-43.1$ & $\begin{array}{l}\text { Reading less than } \\
\text { 1"wg vacuum }\end{array}$ & $\begin{array}{l}\text { Faulty connector, step 1- } \\
\text { 4lomitted }\end{array}$ & None & $\begin{array}{l}\text { Operational upset, Unable to provide good inerting oxygen } \\
\text { monitoring when inerting at end of operation. }\end{array}$ & N/A & N/A & $\begin{array}{l}\text { Leaky connection may cause poor } \\
\text { inerting result if not detected during } \\
\text { connection. } \\
\text { Add step to vacate trailer and platform. } \\
\text { Nobody can be on trailer when tare } \\
\text { weights are measured. } \\
\end{array}$ \\
\hline $1-44$ & \multicolumn{7}{|c|}{ Record first STSC tare weight and level W1 and L1 (WIT-740-401 and LIT-740-401). Initialize PNL-401 by pressing 'high tare' pushbutton HS-403. (Safety significant Measurement) } \\
\hline $1-44.1$ & $\begin{array}{l}\text { Record weight } \\
\text { with person on } \\
\text { trailer }\end{array}$ & Error & None & $\begin{array}{l}\text { Operational upset Future weights biased to allow too much } \\
\text { sludge }\end{array}$ & N/A & N/A & Wrong recordings evaluated in HAZOP \\
\hline
\end{tabular}


Table D-1 "What/If” HAZARDS ANALYSIS RESULTS- OPERATION SEQUENCES (GAP ANALYSIS)

\begin{tabular}{|c|c|c|c|c|c|c|c|}
\hline \multirow[t]{2}{*}{ Identifier* } & \multirow[t]{2}{*}{ "What/ff" } & \multirow[t]{2}{*}{ Causes } & \multirow{2}{*}{$\begin{array}{l}\text { Hazardous } \\
\text { Condition }\end{array}$} & \multirow[t]{2}{*}{ Consequences } & \multicolumn{2}{|c|}{ Candidate Controls } & \multirow[t]{2}{*}{ Remarks/Actions } \\
\hline & & & & & Engineered Features & Admin Controls & \\
\hline $1-45$ & \multicolumn{7}{|c|}{ Verify AOV-201 is in 'filter' position (HS-201 'filter') Verify IL- 201 indicates 'filter' Open V-202 } \\
\hline $1-45.1$ & $\begin{array}{l}\text { Position not set to } \\
\text { filter. }\end{array}$ & Error & None & $\begin{array}{l}\text { Operational upset, } \\
\text { Water will recirculate back to STSC }\end{array}$ & N/A & N/A & \\
\hline $1-45.2$ & $\begin{array}{l}\text { V-202 fails to } \\
\text { open }\end{array}$ & Error & None & Operational upset, suction flow blocked, & N/A & N/A & \\
\hline & \multicolumn{7}{|c|}{ Start decant pump P-201 by opening AOV-760 (HS-760 'start') Verify IL-761 indicates 'running' } \\
\hline $1-46$ & $\begin{array}{l}\text { pump does not } \\
\text { function }\end{array}$ & Pump diaphragm failure & None & \begin{tabular}{|l|} 
Operational upset \\
Can't remove water from STSC
\end{tabular} & N/A & N/A & \\
\hline $1-46.1$ & P-201 sucks air & $\begin{array}{l}\text { V-205 not closed during } \\
\text { hookup }\end{array}$ & None & $\begin{array}{l}\text { Operational upset } \\
\text { Can't remove water from STSC }\end{array}$ & N/A & N/A & \\
\hline $1-47$ & \multicolumn{7}{|c|}{ STSC level drops, resetting interlock I-3. Verify IL-13 and IL- 402 turn OFF. } \\
\hline $1-47.1$ & $\begin{array}{l}\text { Interlock does not } \\
\text { reset. }\end{array}$ & I\&C fault & None & Operational upset & $\mathrm{N} / \mathrm{A}$ & N/A & \\
\hline $1-47.2$ & $\begin{array}{l}\text { IXM water flows } \\
\text { out blank } \\
\text { connector }\end{array}$ & $\begin{array}{l}\text { V-731 not closed due to missed } \\
\text { step 1-38 above }\end{array}$ & $\begin{array}{l}\text { Spread of } \\
\text { contamination }\end{array}$ & $\begin{array}{l}\text { Operational upset, RadCon upset Water on STSC and into } \\
\text { STS. If valve V-731 not closed at } 1-38 \text {, IXM water flow that } \\
\text { was stopped by interlock will flow through open hose onto } \\
\text { STSC, STS and operators }\end{array}$ & N/A & N/A & Consider capping hose. \\
\hline $1-48$ & \multicolumn{7}{|c|}{ 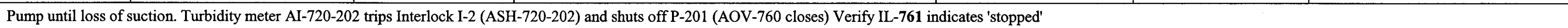 } \\
\hline $1-48.1$ & $\begin{array}{l}\text { not shut down by } \\
\text { turbidity meter }\end{array}$ & $\begin{array}{l}\text { Turbidity meter not valved in or } \\
\text { failed low reading }\end{array}$ & None & $\begin{array}{l}\text { Operational upset } \\
\text { pump runs dry, fails diaphragm }\end{array}$ & N/A & N/A & \\
\hline $1-49$ & \multicolumn{7}{|c|}{ Record second STSC tare weight and level W2 and L2(WIT-740-401 and LIT-740-401). Initialize PNL-401 by pressing 'low tare' pushbutton HS-406. Calculate and verify STSC geometry factor. (Safety Significant Measurement) } \\
\hline $1-49.1$ & $\begin{array}{l}\text { Low tare not set/ } \\
\text { geometry factor } \\
\text { not calculated. }\end{array}$ & Error & None & $\begin{array}{l}\text { Operational upset } \\
\text { Hand calculation and instrument calculation will not be in } \\
\text { synch }\end{array}$ & N/A & N/A & Recording errors identified in HAZOP. \\
\hline $1-49.2$ & $\begin{array}{l}\text { Record weight } \\
\text { with person on } \\
\text { trailer }\end{array}$ & Error & Overpressure & $\begin{array}{l}\text { Potential to overfill container, beyond analyzed value, leading } \\
\text { to reduced transportation window and potential for cask } \\
\text { overpressure, potential worker and public dose }\end{array}$ & None & \begin{tabular}{|l} 
Procedures \\
Training \\
Conduct of operations
\end{tabular} & $\begin{array}{l}\text { This anticipates overfill with settler } \\
\text { sludge shortening the transport window } \\
\text { Recording errors identified in HAZOP. } \\
\text { No one should be on trailer after step 1- } \\
43 \text {. }\end{array}$ \\
\hline & & & & & & & \\
\hline
\end{tabular}


Table D-1 "What/If" HAZARDS ANALYSIS RESULTS- OPERATION SEQUENCES (GAP ANALYSIS)

\begin{tabular}{|c|c|c|c|c|c|c|c|}
\hline \multirow[t]{2}{*}{ Identifier* } & \multirow[t]{2}{*}{ "What/If" } & \multirow[t]{2}{*}{ Causes } & \multirow{2}{*}{$\begin{array}{l}\text { Hazardous } \\
\text { Condition }\end{array}$} & \multirow[t]{2}{*}{ Consequences } & \multicolumn{2}{|c|}{ Candidate Controls } & \multirow[t]{2}{*}{ Remarks/Actions } \\
\hline & & & & & Engineered Features & Admin Controls & \\
\hline $1-50$ & \multicolumn{7}{|c|}{ Secure from 'test' mode } \\
\hline $1-50.1$ & $\begin{array}{l}\text { Not secured from } \\
\text { "test" means that } \\
\text { auto flush from } \\
\text { tank not actuated }\end{array}$ & Error & $\begin{array}{l}\text { Spray leak } \\
\text { Splash and splatter }\end{array}$ & $\begin{array}{l}\text { Operational upset, potential to plug line in loss of power } \\
\text { transfer shut down, overpressure on restart: blow rupture disc } \\
\text { or spray leak, worker or public dose }\end{array}$ & $\begin{array}{l}\text { Rupture discs, } \\
\text { Line design pressure } \\
\text { Secondary confinement } \\
\text { Leak detection } \\
\text { Block/ check valves }\end{array}$ & $\begin{array}{l}\text { Procedures } \\
\text { Training } \\
\text { Conduct of operations }\end{array}$ & $\begin{array}{l}\text { This anticipates a worst case blockage } \\
\text { from a failure to bladder tank flush when } \\
\text { called upon at a future time. }\end{array}$ \\
\hline $1-51$ & \multicolumn{7}{|c|}{ Close V-109. Open V-101 and visually verify alignment of all manual valves in TLSB (V-101,V-103 OPEN, V-102 in 'transfer' position, all others closed). Verify IL-125 is OFF. } \\
\hline $1-51.1$ & $\begin{array}{l}\text { Valve mis- } \\
\text { positioned Both } \\
\text { Valves not closed }\end{array}$ & Error & $\begin{array}{l}\text { Uncontrolled Release } \\
\text { Leak sludge to } \\
\text { transfer box. }\end{array}$ & $\begin{array}{l}\text { Operational upset, RadCon upset with sludge in transfer box } \\
\text { Potential routing of sludge to valve box, trip leak detector, } \\
\text { interlock shutdown pump }\end{array}$ & N/A & N/A & $\begin{array}{l}\text { This anticipates a misdirected flow at a } \\
\text { time during transfer if both valves are left } \\
\text { open. }\end{array}$ \\
\hline $1-51.2$ & $\begin{array}{l}\text { Valve mis- } \\
\text { positioned - } \\
\text { Valve not opened }\end{array}$ & Error & $\begin{array}{l}\text { Spray leak } \\
\text { Splash and splatter }\end{array}$ & $\begin{array}{l}\text { Operational upset. Potential for Worker and public dose; } \\
\text { Deadhead transfer pump when started at step 2-7, potential } \\
\text { overpressure, spray leak. Or blow rupture disc and cloud basin } \\
\text { water with discharged sludge }\end{array}$ & $\begin{array}{l}\text { Rupture discs and interlock } \\
\text { Primary line design } \\
\text { pressure } \\
\text { Secondary confinement } \\
\text { Leak detection } \\
\text { Block/check valves }\end{array}$ & $\begin{array}{l}\text { Procedures } \\
\text { Training } \\
\text { Conduct of operations }\end{array}$ & $\begin{array}{l}\text { This anticipates a line blockage and } \\
\text { overpressure when the booster pump is } \\
\text { started. } \\
\text { V-102 not an "open" valve (it is a 3-way } \\
\text { valve) as indicate in step description. } \\
\text { Dead head/overpressure identified in } \\
\text { HAZOP }\end{array}$ \\
\hline $1-51.2$ & Light is not off & Incorrect circuit & None & Operational upset. repair & & & \\
\hline $1-52$ & \multicolumn{7}{|c|}{ Close V-205. Open V-202, and visually verify alignment of all manual valves in decant box (V-201, V-202, V-204, V-210, V- 227 OPEN, all others closed) IL-223 is OFF. } \\
\hline $1-52.1$ & $\begin{array}{l}\text { Valve mis- } \\
\text { positioned V-205 } \\
\text { left open }\end{array}$ & Error & None & $\begin{array}{l}\text { Operational upset, potential Radcon upset } \\
\text { Future misroute of flow to decant box or suck air at decant } \\
\text { pump }\end{array}$ & $\mathrm{N} / \mathrm{A}$ & N/A & \\
\hline $1-52.2$ & $\begin{array}{l}\text { Valve not } \\
\text { opened. }\end{array}$ & Error & $\begin{array}{l}\text { Spray leak } \\
\text { Splash and splatter }\end{array}$ & $\begin{array}{l}\text { Operational upset; or worker and public dose } \\
\text { Decant or recirc not aligned, pump dead headed }\end{array}$ & $\begin{array}{l}\text { Limited pump discharge } \\
\text { pressure } \\
\text { Primary line design } \\
\text { pressure, } \\
\text { secondary confinement } \\
\text { leak detection } \\
\text { Block/check valves } \\
\end{array}$ & $\begin{array}{l}\text { Procedures } \\
\text { Training } \\
\text { Conduct of operations }\end{array}$ & $\begin{array}{l}\text { V-227 intended to be a throttle valve, not } \\
\text { "open" }\end{array}$ \\
\hline $1-52.2$ & Light is not off & Incorrect circuit & None & Operational upset. repair & & & \\
\hline $1-53$ & \multicolumn{6}{|c|}{ Exit and secure Annex loading bay } & \\
\hline
\end{tabular}


Table D-1 "What/If" HAZARDS ANALYSIS RESULTS- OPERATION SEQUENCES (GAP ANALYSIS)

\begin{tabular}{|c|c|c|c|c|c|c|c|}
\hline \multirow[t]{2}{*}{ Identifier* } & \multirow[t]{2}{*}{ "What/If" } & \multirow[t]{2}{*}{ Causes } & \multirow{2}{*}{$\begin{array}{l}\text { Hazardous } \\
\text { Condition }\end{array}$} & \multirow[t]{2}{*}{ Consequences } & \multicolumn{2}{|c|}{ Candidate Controls } & \multirow[t]{2}{*}{ Remarks/Actions } \\
\hline & & & & & Engineered Features & Admin Controls & \\
\hline $1-53-1$ & $\begin{array}{l}\text { Not everyone } \\
\text { leaves }\end{array}$ & Error & Direct dose & $\begin{array}{l}\text { RadCon Upset } \\
\text { Potential high dose during transfer }\end{array}$ & Shielded lines & \begin{tabular}{|l} 
Procedures \\
Training \\
Conduct of operations \\
Administrative \\
restriction on access to \\
annex as part of RadCon \\
program
\end{tabular} & \\
\hline \multicolumn{7}{|c|}{ Mode 8 DISCONNECT STSC } & \\
\hline $8-1$ & \multicolumn{6}{|c|}{ Enter Annex loading bay } & \\
\hline $8-1.1$ & $\begin{array}{l}\text { Airborne } \\
\text { contamination }\end{array}$ & Leaked sludge material & $\begin{array}{l}\text { Spread of } \\
\text { contamination }\end{array}$ & Operational upset, Radcon event, Recovery plan & $\begin{array}{l}\text { Primary confinement } \\
\text { Secondary confinement } \\
\text { Leak detection } \\
\text { Ventilation zones }\end{array}$ & \begin{tabular}{|l|} 
RadCon monitoring \\
Procedures \\
Training \\
Conduct of operations
\end{tabular} & \\
\hline $8-1.2$ & $\begin{array}{l}\text { Elevated direct } \\
\text { dose rates }\end{array}$ & $\begin{array}{l}\text { Lines not flushed or leaked } \\
\text { material }\end{array}$ & $\begin{array}{l}\text { Personnel direct dose } \\
\text { Spread of } \\
\text { contamination }\end{array}$ & Operational upset, Radcon event, Recovery plan & $\begin{array}{l}\text { Primary confinement } \\
\text { Secondary confinement } \\
\text { Leak detection } \\
\text { Ventilation zones }\end{array}$ & \begin{tabular}{|l|} 
RadCon monitoring \\
Procedures \\
Training \\
Conduct of operations \\
\end{tabular} & \\
\hline $8-1.3$ & $\begin{array}{l}\text { Ventilation not } \\
\text { running }\end{array}$ & Ventilation system fault & Industrial hazard & $\begin{array}{l}\text { Operational upset, Recovery plan. Assume not habitable from } \\
\text { industrial or Radcon perspective. }\end{array}$ & $\mathrm{N} / \mathrm{A}$ & N/A & \\
\hline $8-2$ & \multicolumn{7}{|c|}{ Perform final IXM flush of sludge line: Close V-101, open AOV-104 } \\
\hline $8-2.1$ & $\begin{array}{l}\text { Valve-101 not } \\
\text { closed }\end{array}$ & Step skipped, error & None & $\begin{array}{l}\text { Operational upset, Recovery plan } \\
\text { May get bladder flush, excess fill of STSC }\end{array}$ & N/A & N/A & $\begin{array}{l}\text { Requires access to transfer box. } \\
\text { Need to consider piping modification to } \\
\text { enable remote IXM flush before entering } \\
\text { Annex. }\end{array}$ \\
\hline $8-2.2$ & $\begin{array}{l}\text { Valves- } 712 \text { and } \\
\text { AOV-104 not } \\
\text { opened }\end{array}$ & Step skipped, error & Personnel direct dose & $\begin{array}{l}\text { Operational upset, Recovery plan Flush not performed, higher } \\
\text { dose rates during access }\end{array}$ & N/A & N/A & $\begin{array}{l}\text { Need to consider piping modification to } \\
\text { enable remote IXM flush before entering } \\
\text { Annex. }\end{array}$ \\
\hline $8-3$ & \multicolumn{7}{|c|}{ Secure from final sludge line flush: Close AOV-104 } \\
\hline $8-3.1$ & Valves not closed & Step skipped, error & None & $\begin{array}{l}\text { Operational upset, recovery plan Flush continues, interlock } 3 \\
\text { shuts down flush }\end{array}$ & $\mathrm{N} / \mathrm{A}$ & N/A & \\
\hline $8-3.2$ & Valves not closed & Step skipped, error & $\begin{array}{l}\text { Spread of } \\
\text { contamination }\end{array}$ & $\begin{array}{l}\text { Operational upset, radcon upset } \\
\text { Next step potentially introduces liquid to transfer box. }\end{array}$ & N/A & \begin{tabular}{|l} 
Procedures \\
Training \\
Conduct of operations
\end{tabular} & \\
\hline $8-4$ & \multicolumn{7}{|c|}{ Drain sludge line: Open V-109 } \\
\hline
\end{tabular}


Table D-1 "What/If" HAZARDS ANALYSIS RESULTS- OPERATION SEQUENCES (GAP ANALYSIS)

\begin{tabular}{|c|c|c|c|c|c|c|c|}
\hline \multirow[t]{2}{*}{ Identifier* } & \multirow[t]{2}{*}{ "What/ff" } & \multirow[t]{2}{*}{ Causes } & \multirow{2}{*}{$\begin{array}{l}\text { Hazardous } \\
\text { Condition }\end{array}$} & \multirow[t]{2}{*}{ Consequences } & \multicolumn{2}{|c|}{ Candidate Controls } & \multirow[t]{2}{*}{ Remarks/Actions } \\
\hline & & & & & Engineered Features & Admin Controls & \\
\hline $8-4.1$ & $\begin{array}{l}\text { Valve not } \\
\text { operated }\end{array}$ & & $\begin{array}{l}\text { Spread of } \\
\text { contamination }\end{array}$ & $\begin{array}{l}\text { Operational upset, RadCon upset, } \\
\text { Line remains with liquid in it when disconnected }\end{array}$ & $\mathrm{N} / \mathrm{A}$ & $\begin{array}{l}\text { Procedures } \\
\text { Training } \\
\text { Conduct of operations }\end{array}$ & \\
\hline $8-5$ & \multicolumn{7}{|c|}{ Perform final Decant/backwash line flush: Close V-202, Open V-206 } \\
\hline $8-5.1$ & $\begin{array}{l}\text { V-202 not closed, } \\
\text { V-206 opened }\end{array}$ & Performance error & None & \begin{tabular}{|l|} 
Operational upset \\
Flush both directions: to STSC and through filters
\end{tabular} & N/A & N/A & V-206 open all the way is a large flow. \\
\hline $8-6$ & \multicolumn{7}{|c|}{ Top off STSC with IXM water to final level for shipping using V-206 } \\
\hline $8-6.1$ & Not topped off & Step skipped & Personnel dose & $\begin{array}{l}\text { RadCon upset } \\
\text { Dose not minimized with maximum shielding }\end{array}$ & N/A & $\begin{array}{l}\text { Procedures } \\
\text { Training } \\
\text { Conduct of operations } \\
\text { RadCon Monitoring }\end{array}$ & $\begin{array}{l}\text { Not clear why previous step did not } \\
\text { accomplish this action. }\end{array}$ \\
\hline $8-7$ & \multicolumn{7}{|c|}{ Secure from final Decant/backwash line flush: Close V-206 } \\
\hline $8-7.1$ & Valves not closed & Performance error & None & $\begin{array}{l}\text { Operational upset } \\
\text { Flush continues, interlock } 3 \text { shuts down flush }\end{array}$ & N/A & N/A & \\
\hline $8-8$ & \multicolumn{7}{|c|}{ Drain decant/backwash line: Open V-205 } \\
\hline $8-8.1$ & $\begin{array}{l}\text { Step not done, } \\
\text { line not drained }\end{array}$ & Performance error & $\begin{array}{l}\text { spread of } \\
\text { contamination }\end{array}$ & $\begin{array}{l}\text { RadCon Upset, Decant/backwash line retains water for } \\
\text { disconnect error, see 8-18.1 }\end{array}$ & N/A & $\begin{array}{l}\text { Procedures } \\
\text { Training } \\
\text { Conduct of operations }\end{array}$ & \\
\hline $8-9$ & \multicolumn{7}{|c|}{ Record STSC Weight and level(WIT-740-401 and LIT-740-401). Calculate and verify final buoyant weight of sludge transferred (DWI-740-401). Begin preparation of shipping manifest. (Safety Significant Measurement) } \\
\hline $8-9.1$ & Record wrongly & Performance error & Covered in HAZOP & & N/A & N/A & $\begin{array}{l}\text { There is also an automatic recording of } \\
\text { weight and level. } \\
\text { Nobody should be allowed on the truck } \\
\text { until this step is completed. }\end{array}$ \\
\hline $8-9.2$ & $\begin{array}{l}\text { Calculate } \\
\text { wrongly }\end{array}$ & Performance error & Covered in HAZOP & & $\mathrm{N} / \mathrm{A}$ & N/A & $\begin{array}{l}\text { There is also an automatic calculation } \\
\text { and recording }\end{array}$ \\
\hline $8-10$ & \multicolumn{7}{|c|}{ Unbolt the Sludge Inlet Connection. Raise flange 2" to open the connector internal vent port. Verify IL-1 18 turns OFF. } \\
\hline
\end{tabular}


Table D-1 "What/If" HAZARDS ANALYSIS RESULTS- OPERATION SEQUENCES (GAP ANALYSIS)

\begin{tabular}{|c|c|c|c|c|c|c|c|}
\hline \multirow[t]{2}{*}{ Identifier* } & \multirow[t]{2}{*}{ "What/If" } & \multirow[t]{2}{*}{ Causes } & \multirow{2}{*}{$\begin{array}{l}\text { Hazardous } \\
\text { Condition }\end{array}$} & \multirow[t]{2}{*}{ Consequences } & \multicolumn{2}{|c|}{ Candidate Controls } & \multirow{2}{*}{ Remarks/Actions } \\
\hline & & & & & Engineered Features & Admin Controls & \\
\hline $8-10.1$ & $\begin{array}{l}\text { Not disconnected } \\
\text { when lifted }\end{array}$ & Step not performed properly & None & $\begin{array}{l}\text { Operational upset, recovery plan Potential to damage hose, } \\
\text { break hose, damage bail }\end{array}$ & N/A & N/A & \\
\hline $8-10.2$ & $\begin{array}{l}\text { Lift too high, } \\
\text { drips }\end{array}$ & Step not performed properly & $\begin{array}{l}\text { Spread of } \\
\text { contamination }\end{array}$ & $\begin{array}{l}\text { Operational upset, RadCon event } \\
\text { Contaminated liquid drips on top of STSC }\end{array}$ & N/A & \begin{tabular}{|l|} 
Procedures \\
Training \\
Conduct of operations
\end{tabular} & \\
\hline $8-10.3$ & $\begin{array}{l}\text { Lift wrong } \\
\text { hose/flange }\end{array}$ & Step not performed properly & $\begin{array}{l}\text { Spread of } \\
\text { contamination }\end{array}$ & $\begin{array}{l}\text { Operational upset, RadCon event } \\
\text { Potential to damage hose or bail, leak contamination }\end{array}$ & N/A & \begin{tabular}{|l|} 
Procedures \\
Training \\
Conduct of operations \\
\end{tabular} & \\
\hline $8-11$ & \multicolumn{7}{|c|}{ Verify positive airflow through TLSB high point vent (IL-223 turns ON) } \\
\hline 8-11.1 & \begin{tabular}{|l|} 
No flow \\
indication
\end{tabular} & $\begin{array}{l}\text { Failed element, failed indicator, } \\
\text { V-201 not open }\end{array}$ & None & $\begin{array}{l}\text { Operational upset, Recovery plan required } \\
\text { Line may not be drained down, or draining slowly }\end{array}$ & $\mathrm{N} / \mathrm{A}$ & $\mathrm{N} / \mathrm{A}$ & \\
\hline $8-12$ & \multicolumn{7}{|c|}{ Unbolt the Decant/backwash line Connection. Raise flange 2" to open the connector internal vent port. Verify IL-218 turns OFF. } \\
\hline $8-12.1$ & \begin{tabular}{|l|}
$\begin{array}{l}\text { Not disconnected } \\
\text { when lifted }\end{array}$ \\
\end{tabular} & Step not performed properly & None & $\begin{array}{l}\text { Operational upset, recovery plan Potential to damage hose, } \\
\text { break hose, damage bail }\end{array}$ & N/A & $\mathrm{N} / \mathrm{A}$ & \\
\hline $8-12.2$ & $\begin{array}{l}\text { Liff too high, } \\
\text { drips }\end{array}$ & Step not performed properly & $\begin{array}{l}\text { Spread of } \\
\text { contamination }\end{array}$ & $\begin{array}{l}\text { Operational upset, RadCon event } \\
\text { Contaminated liquid drips on STSC }\end{array}$ & N/A & \begin{tabular}{|l|} 
Procedures \\
Training \\
Conduct of operations
\end{tabular} & \\
\hline $8-12.3$ & $\begin{array}{l}\text { Lift wrong } \\
\text { hose/flange }\end{array}$ & Step not performed properly & $\begin{array}{l}\text { Spread of } \\
\text { contamination }\end{array}$ & $\begin{array}{l}\text { Operational upset, potential RadCon event } \\
\text { Potential to damage hose or bail, or release contamination }\end{array}$ & N/A & \begin{tabular}{|l|} 
Procedures \\
Training \\
Conduct of operations \\
\end{tabular} & \\
\hline $8-13$ & \multicolumn{7}{|c|}{ Verify positive airflow through Decant box high point vent (IL- 125 turns ON) } \\
\hline $8-13.1$ & \begin{tabular}{|l|l|} 
No flow \\
indication
\end{tabular} & \begin{tabular}{|l} 
Failed element, failed indicator, \\
V-205 not open \\
\end{tabular} & None & $\begin{array}{l}\text { Operational upset, Recovery plan required } \\
\text { Line may not be drained down, or draining slowly }\end{array}$ & N/A & N/A & \\
\hline $8-14$ & \multicolumn{7}{|c|}{$\begin{array}{l}\text { Note: At this point, air is flowing from the room into the decant box and the TLSB through the HIH annulus, and from the air breaks in the decant box and TLSB through the inner hose of HIH hoses H-107 and H-203 into the STSC. Leave nozzles } \\
\text { in this configuration until the inner hoses are drip-free. }\end{array}$} \\
\hline $8-14.1$ & N/A & N/A & N/A & $\mathrm{N} / \mathrm{A}$ & N/A & N/A & \\
\hline $8-15$ & \multicolumn{7}{|c|}{ Disconnect the Sludge line connector from Nozzle A. Install 3- bolt blind flange on Nozzle A. } \\
\hline
\end{tabular}


Table D-1 "What/If" HAZARDS ANALYSIS RESULTS- OPERATION SEQUENCES (GAP ANALYSIS)

\begin{tabular}{|c|c|c|c|c|c|c|c|}
\hline \multirow[t]{2}{*}{ Identifier* } & \multirow[t]{2}{*}{ "What/If" } & \multirow[t]{2}{*}{ Causes } & \multirow{2}{*}{$\begin{array}{l}\text { Hazardous } \\
\text { Condition }\end{array}$} & \multirow[t]{2}{*}{ Consequences } & \multicolumn{2}{|c|}{ Candidate Controls } & \multirow[t]{2}{*}{ Remarks/Actions } \\
\hline & & & & & Engineered Features & Admin Controls & \\
\hline 8-15.1 & $\begin{array}{l}\text { Drop hose and } \\
\text { damage nozzle or } \\
\text { impact personnel }\end{array}$ & Rigging or crane operation error & Industrial hazard & Operational upset, personnel injury, delay for repair & N/A & N/A & \\
\hline $8-15.2$ & $\begin{array}{l}\text { Lift hose out of } \\
\text { glove bag }\end{array}$ & Crane operation error & $\begin{array}{l}\text { spread of } \\
\text { contamination }\end{array}$ & Operational upset. Delay for RadCon recovery, & N/A & $\begin{array}{l}\text { Procedures } \\
\text { Training } \\
\text { Conduct of operations }\end{array}$ & \\
\hline 8-16 & \multicolumn{7}{|c|}{ Disconnect the Decant/backwash line connector from Nozzle B. Install 4-bolt blind flange on Nozzle B. } \\
\hline $8-16.1$ & $\begin{array}{l}\text { Drop hose and } \\
\text { damage nozzle or } \\
\text { impact personnel }\end{array}$ & Rigging or crane operation error & Industrial hazard & Operational upset, Injure personnel, recovery plan & N/A & N/A & \\
\hline $8-16.2$ & $\begin{array}{l}\text { Lift hose out of } \\
\text { glove bag }\end{array}$ & Crane operation error & $\begin{array}{l}\text { Spread of } \\
\text { contamination }\end{array}$ & Operational upset, delay for RadCon recovery & N/A & $\begin{array}{l}\text { Procedures } \\
\text { Training } \\
\text { Conduct of operations }\end{array}$ & \\
\hline 8-17 & \multicolumn{7}{|c|}{ Note: At this point, air is flowing from the room up through both the inner hose and the annulus of $\mathrm{H}-107$ and $\mathrm{H}-203$ into the TLSB and the decant box } \\
\hline $8-17.1$ & N/A & N/A & N/A & N/A & N/A & $\mathrm{N} / \mathrm{A}$ & $\begin{array}{l}\text { This step will be evaluated at MASF } \\
\text { since dry hose may lead to fugitive } \\
\text { emissions. }\end{array}$ \\
\hline $8-18$ & \multicolumn{7}{|c|}{ Install the end caps and contamination controls on the sludge and decant/backwash line connectors. Place the connectors in the storage position. } \\
\hline $8-18.1$ & $\begin{array}{l}\begin{array}{l}\text { Some liquid } \\
\text { drops from lines }\end{array} \\
\end{array}$ & $\begin{array}{l}\text { Thermal expansion of liquid in } \\
\text { system, line not dry }\end{array}$ & $\begin{array}{l}\text { Spread of } \\
\text { contamination }\end{array}$ & Operational upset, RadCon recovery & N/A & None & Plan to be working in a glove bag. \\
\hline $8-19$ & \multicolumn{7}{|c|}{ Secure from Sludge line draining/venting: Close V-109 } \\
\hline 8-19.1 & N/A & $\mathrm{N} / \mathrm{A}$ & N/A & N/A & N/A & $\mathrm{N} / \mathrm{A}$ & $\begin{array}{l}\text { If not closed, may be an issue in future } \\
\text { valve alignment, but HVAC sucking on } \\
\text { hose via box and open valve. }\end{array}$ \\
\hline $8-20$ & \multicolumn{7}{|c|}{ Secure from Decant/backwash line draining/venting: Close V-205 } \\
\hline $8-20.1$ & $\mathrm{~N} / \mathrm{A}$ & $\mathrm{N} / \mathrm{A}$ & N/A & N/A & N/A & N/A & $\begin{array}{l}\text { If not closed, may be an issue in future } \\
\text { valve alignment, but HVAC sucking on } \\
\text { hose via box and open valve. }\end{array}$ \\
\hline
\end{tabular}


Table D-1 "What/If" HAZARDS ANALYSIS RESULTS- OPERATION SEQUENCES (GAP ANALYSIS)

\begin{tabular}{|c|c|c|c|c|c|c|c|}
\hline \multirow[t]{2}{*}{ Identifier* } & \multirow[t]{2}{*}{ "What/ff" } & \multirow[t]{2}{*}{ Causes } & \multirow{2}{*}{$\begin{array}{l}\text { Hazardous } \\
\text { Condition }\end{array}$} & \multirow[t]{2}{*}{ Consequences } & \multicolumn{2}{|c|}{ Candidate Controls } & \multirow[t]{2}{*}{ Remarks/Actions } \\
\hline & & & & & Engineered Features & Admin Controls & \\
\hline $8-21$ & \multicolumn{7}{|c|}{ Disconnect the 4 inch STSC Vent connection and install the Camlok cap on Nozzle F1 } \\
\hline $8-21.1$ & $\begin{array}{l}\text { Drop line and } \\
\text { damage nozzle, } \\
\text { impact personnel }\end{array}$ & Rigging or crane operation error & Industrial hazard & Operational upset, personnel injury, recovery plan & $\mathrm{N} / \mathrm{A}$ & N/A & \\
\hline $8-21.2$ & $\begin{array}{l}\text { Lift hose out of } \\
\text { glove bag }\end{array}$ & Crane operation error & $\begin{array}{l}\text { Spread of } \\
\text { contamination }\end{array}$ & Operational upset, delay for RadCon recovery & N/A & $\begin{array}{l}\text { Procedures } \\
\text { Training } \\
\text { Conduct of operations } \\
\end{array}$ & May not have glove bag \\
\hline $8-22$ & \multicolumn{7}{|c|}{ Initiate STSC Inert Gas Purge: } \\
\hline $8-22.1$ & $\begin{array}{l}\text { Covered in } \\
\text { HAZOP }\end{array}$ & N/A & N/A & $\mathrm{N} / \mathrm{A}$ & N/A & N/A & \\
\hline $8-23$ & \multicolumn{7}{|c|}{ Align inert gas 3-way valve V-609 to 'STSC' position } \\
\hline 8-23.1 & $\begin{array}{l}\text { Covered in } \\
\text { HAZOP }\end{array}$ & N/A & $\mathrm{N} / \mathrm{A}$ & N/A & N/A & N/A & \\
\hline $8-24$ & \multicolumn{7}{|c|}{ Open V-608. Inert gas flows into the STSC. } \\
\hline 8-24.1 & $\begin{array}{l}\text { Covered in } \\
\text { HAZOP }\end{array}$ & N/A & N/A & $\mathrm{N} / \mathrm{A}$ & N/A & N/A & \\
\hline $8-25$ & \multicolumn{7}{|c|}{ Verify/adjust inert gas flow rate with rotameter FI-760-601 } \\
\hline $8-25.1$ & $\begin{array}{l}\text { Covered in } \\
\text { HAZOP }\end{array}$ & N/A & $\mathrm{N} / \mathrm{A}$ & $\mathrm{N} / \mathrm{A}$ & N/A & N/A & \\
\hline $8-26$ & \multicolumn{7}{|c|}{ Close V-732. Air purge flow stops. Verify IL-651 is OFF. } \\
\hline $8-26.1$ & $\begin{array}{l}\text { Covered in } \\
\text { HAZOP } \\
\end{array}$ & N/A & N/A & $\mathrm{N} / \mathrm{A}$ & N/A & N/A & \\
\hline $8-27$ & \multicolumn{7}{|c|}{ Prior to securing from STSC Inerting, insure shipping window availability. } \\
\hline $8-27.1$ & $\begin{array}{l}\text { Covered in } \\
\text { HAZOP }\end{array}$ & N/A & N/A & $\mathrm{N} / \mathrm{A}$ & $\mathrm{N} / \mathrm{A}$ & $\mathrm{N} / \mathrm{A}$ & \\
\hline & & & & & & & \\
\hline
\end{tabular}


Table D-1 "What/If" HAZARDS ANALYSIS RESULTS- OPERATION SEQUENCES (GAP ANALYSIS)

\begin{tabular}{|c|c|c|c|c|c|c|c|}
\hline \multirow[t]{2}{*}{ Identifier ${ }^{*}$} & \multirow[t]{2}{*}{ "What/ff" } & \multirow[t]{2}{*}{ Causes } & \multirow{2}{*}{$\begin{array}{l}\text { Hazardous } \\
\text { Condition }\end{array}$} & \multirow[t]{2}{*}{ Consequences } & \multicolumn{2}{|c|}{ Candidate Controls } & \multirow[t]{2}{*}{ Remarks/Actions } \\
\hline & & & & & Engineered Features & Admin Controls & \\
\hline $8-28$ & \multicolumn{7}{|c|}{ Secure from STSC Inert Gas Purge upon O2 Sensor AI-760-601 reading less than 1 vol\%: Close V-608.. Start the Cask Purge Clock. } \\
\hline 8-28.1 & $\begin{array}{l}\text { Covered in } \\
\text { HAZOP }\end{array}$ & N/A & N/A & N/A & N/A & N/A & $\begin{array}{l}\text { Note: If V-608 is not closed, purge flow } \\
\text { would continue after disconnecting purge } \\
\text { outlet connection (Step } 8-30 \text { ) and STSC } \\
\text { could pressurize up to } 65 \text { psig. This is } \\
\text { less than the design pressure of } 150 \text { psig. } \\
\text { This will only create a hazard upon } \\
\text { inserting of STSC vent filter which } \\
\text { would fail and depressurize the STSC } \\
\text { above a dp of approximately } 9 \text { psi. }\end{array}$ \\
\hline $8-29$ & \multicolumn{7}{|c|}{ Disconnect the Purge Outlet and install shipping Filter onto the 2 inch Quick Connect Nozzle F2 } \\
\hline 8-29.1 & $\begin{array}{l}\text { Fail to install } \\
\text { filter }\end{array}$ & Skip step or error & $\begin{array}{l}\text { Spread of } \\
\text { contamination } \\
\text { Deflagration }\end{array}$ & $\begin{array}{l}\text { RadCon event } \\
\text { Inerting degraded due to air exchange, hydrogen } \\
\text { accumulation, worker and public dose }\end{array}$ & None & $\begin{array}{l}\text { Procedures } \\
\text { Training } \\
\text { Conduct of operations } \\
\text { Administrative window }\end{array}$ & $\begin{array}{l}\text { Test oxygen inert decay at MASF, affects } \\
\text { duration of "clock" }\end{array}$ \\
\hline 8-29.2 & Take too long & Delay in operation & Deflagration & $\begin{array}{l}\text { Inerting degraded due to air exchange, hydrogen accumulation } \\
\text { worker and public dose }\end{array}$ & None & $\begin{array}{l}\text { Procedures } \\
\text { Training } \\
\text { Conduct of operations } \\
\text { Administrative window }\end{array}$ & $\begin{array}{l}\text { Test oxygen inert decay at MASF, } \\
\text { affects duration of "clock" }\end{array}$ \\
\hline 8-30 & \multicolumn{7}{|c|}{ Disconnect the STSC Purge Gas inlet hose and install the shipping filter onto the 1 inch Quick Connect Nozzle S2 } \\
\hline 8-30.1 & $\begin{array}{l}\text { Fail to install } \\
\text { filter }\end{array}$ & Skip step or error & $\begin{array}{l}\text { Spread of } \\
\text { contamination } \\
\text { Deflagration }\end{array}$ & $\begin{array}{l}\text { RadCon event. } \\
\text { Inerting degraded due to air exchange, hydrogen accumulation } \\
\text {,worker and public dose }\end{array}$ & None & $\begin{array}{l}\text { Procedures } \\
\text { Training } \\
\text { Conduct of operations } \\
\text { Administrative window }\end{array}$ & $\begin{array}{l}\text { Test oxygen inert decay at MASF, affects } \\
\text { duration of "clock" }\end{array}$ \\
\hline 8-30.2 & Take too long & Delay in operation & Deflagration & $\begin{array}{l}\text { Inerting degraded due to air exchange, hydrogen accumulation, } \\
\text { worker and public dose }\end{array}$ & None & $\begin{array}{l}\text { Procedures } \\
\text { Training } \\
\text { Conduct of operations } \\
\text { Administrative window }\end{array}$ & $\begin{array}{l}\text { Test oxygen inert decay at MASF, } \\
\text { affects duration of "clock" }\end{array}$ \\
\hline $8-31$ & \multicolumn{7}{|c|}{ Disconnect I\&C connector to LDE-710-103 (Leak Detector) and LE-740-401 (Level Detector) Safety Significant instruments } \\
\hline 8-31.1 & N/A & N/A & N/A & N/A & N/A & N/A & $\begin{array}{l}\text { Filling and Recording complete. } \\
\text { Add step to turn off instrument } \\
\text { disconnect switch. }\end{array}$ \\
\hline $8-32$ & \multicolumn{7}{|c|}{ onnect I\&C connector $\mathrm{t}$} \\
\hline
\end{tabular}


Table D-1 "What/If" HAZARDS ANALYSIS RESULTS- OPERATION SEQUENCES (GAP ANALYSIS)

\begin{tabular}{|c|c|c|c|c|c|c|c|}
\hline \multirow[t]{2}{*}{ Identifier* } & \multirow[t]{2}{*}{ "What/If" } & \multirow[t]{2}{*}{ Causes } & \multirow{2}{*}{$\begin{array}{l}\text { Hazardous } \\
\text { Condition }\end{array}$} & \multirow[t]{2}{*}{ Consequences } & \multicolumn{2}{|c|}{ Candidate Controls } & \multirow[t]{2}{*}{ Remarks/Actions } \\
\hline & & & & & Engineered Features & Admin Controls & \\
\hline $8-32.1$ & $\mathrm{~N} / \mathrm{A}$ & $\mathrm{N} / \mathrm{A}$ & $\mathrm{N} / \mathrm{A}$ & N/A & $\mathrm{N} / \mathrm{A}$ & N/A & \\
\hline $8-33$ & \multicolumn{7}{|c|}{ Concurrent with Purging operations, Inspect the STS Lid o-ring and replace Helicoflex seal. } \\
\hline 8-33.1 & Skip replace & Error & None & Operational Upset, Lid won't seal. Unable to inert and ship & $\mathrm{N} / \mathrm{A}$ & $\mathrm{N} / \mathrm{A}$ & $\begin{array}{l}\text { Trailer access issue, consider replace seal } \\
\text { at setup }\end{array}$ \\
\hline $8-33.2$ & \begin{tabular}{|l|l|} 
Encounter \\
contamination
\end{tabular} & Previous contamination & None & $\begin{array}{l}\text { Operational Upset, Requires decon prior to maintenance adds } \\
\text { to time against inerting clock. }\end{array}$ & N/A & N/A & \\
\hline $8-34$ & \multicolumn{7}{|c|}{ Install the STS Lid and torque Lid Bolts. } \\
\hline $8-34.1$ & Bolts won't seat & Bad threads & None & $\begin{array}{l}\text { Operational upset, Requires rework, added time against } \\
\text { inerting clock }\end{array}$ & $\mathrm{N} / \mathrm{A}$ & N/A & \\
\hline $8-35$ & \multicolumn{7}{|c|}{ Remove Lid Lift Fixture from STS cask lid } \\
\hline $8-35.1$ & $\begin{array}{l}\text { Drop it, damage } \\
\text { to STS, personnel }\end{array}$ & Rigging error & Industrial hazard & $\begin{array}{l}\text { Personnel injury, Require rework added time against inerting } \\
\text { clock }\end{array}$ & $\mathrm{N} / \mathrm{A}$ & N/A & \\
\hline $8-36$ & \multicolumn{7}{|c|}{ Perform leak test on STS Lid: } \\
\hline $8-37$ & \multicolumn{7}{|c|}{ Remove leak test plug } \\
\hline $8-37.1$ & $\begin{array}{l}\text { Plug won't come } \\
\text { out. }\end{array}$ & Threads damaged & None & $\begin{array}{l}\text { Operational upset Repair/Rework. May need to remove cask } \\
\text { lid and re-inert STSC or purge cask while repairing seal test. }\end{array}$ & $\mathrm{N} / \mathrm{A}$ & N/A & \\
\hline $8-38$ & \multicolumn{7}{|c|}{ Install leak test tool in test port } \\
\hline $8-38.1$ & Tool not installed & Threads damaged & None & $\begin{array}{l}\text { Operational upset, Repair/Rework. May need to remove cask } \\
\text { lid and re-inert STSC or purge cask while repairing seal test. }\end{array}$ & $\mathrm{N} / \mathrm{A}$ & $\mathrm{N} / \mathrm{A}$ & \\
\hline $8-39$ & \multicolumn{7}{|c|}{ Pressurize the lid seal annulus and monitor for specified time } \\
\hline $8-39.1$ & $\begin{array}{l}\text { Step not } \\
\text { performed }\end{array}$ & Error & None & $\begin{array}{l}\text { Cask boundary not verified. Cask not ready to ship. Potential } \\
\text { for cask to leak if a fault occurs. }\end{array}$ & N/A & \begin{tabular}{|l|} 
Procedures \\
Training \\
Conduct of operations \\
\end{tabular} & \\
\hline $8-39.2$ & Fail leak test & & & & & & \\
\hline $8-40$ & \multicolumn{7}{|c|}{ Release pressure and remove leak test tool } \\
\hline
\end{tabular}


Table D-1 "What/If" HAZARDS ANALYSIS RESULTS- OPERATION SEQUENCES (GAP ANALYSIS)

\begin{tabular}{|c|c|c|c|c|c|c|c|}
\hline \multirow[t]{2}{*}{ Identifier* } & \multirow[t]{2}{*}{ "What/If" } & \multirow[t]{2}{*}{ Causes } & \multirow{2}{*}{$\begin{array}{l}\text { Hazardous } \\
\text { Condition }\end{array}$} & \multirow[t]{2}{*}{ Consequences } & \multicolumn{2}{|c|}{ Candidate Controls } & \multirow[t]{2}{*}{ Remarks/Actions } \\
\hline & & & & & Engineered Features & Admin Controls & \\
\hline $8-40.1$ & $\begin{array}{l}\text { Step not } \\
\text { performed }\end{array}$ & Error & None & Operational upset. Tool must be removed to ship. & N/A & N/A & \\
\hline $8-41$ & \multicolumn{7}{|c|}{ Install leak test port plug } \\
\hline $8-41.1$ & \begin{tabular}{|l|}
$\begin{array}{l}\text { Step not } \\
\text { performed }\end{array}$ \\
\end{tabular} & Error, or bad threads & None & $\begin{array}{l}\text { Operational upset, Cask boundary not complete, added time } \\
\text { against inerting clock }\end{array}$ & $\mathrm{N} / \mathrm{A}$ & N/A & \\
\hline \multicolumn{8}{|c|}{ Mode 9 PREPARE FOR SHIPPING } \\
\hline $9-1$ & \multicolumn{7}{|c|}{ Remove STS Cask drain port cap and install drain port tool. } \\
\hline $9-1.1$ & Thread problems & Error or bad threads & None & $\begin{array}{l}\text { Operational upset, Delay start of STS purge, rework, added } \\
\text { time against inerting clock, }\end{array}$ & N/A & $\mathrm{N} / \mathrm{A}$ & Need to check for liquid \\
\hline $9-2$ & \multicolumn{7}{|c|}{ Attach vent line $(\mathrm{H}-659)$ to vent port tool } \\
\hline $9-2.1$ & \begin{tabular}{|l|}
$\begin{array}{l}\text { Not connected or } \\
\text { partially } \\
\text { connected }\end{array}$ \\
\end{tabular} & Error, faulty connector & None & $\begin{array}{l}\text { Operational upset, Delay start of STS purge, rework added } \\
\text { time against inerting clock, }\end{array}$ & N/A & N/A & $\begin{array}{l}\text { Leak of nitrogen identified below in step } \\
9-7 \text {. }\end{array}$ \\
\hline $9-3$ & \multicolumn{7}{|c|}{ Open STS drain port and STS vent port } \\
\hline $9-3.1$ & $\begin{array}{l}\text { STS drain port } \\
\text { not opened }\end{array}$ & Error & None & Operational Upset, No flow to STS at next step & N/A & $\mathrm{N} / \mathrm{A}$ & \\
\hline $9-3.2$ & \begin{tabular}{|l|}
$\begin{array}{l}\text { Vent port not } \\
\text { opened, }\end{array}$ \\
\end{tabular} & Error & None & Operational Upset, Pressurize STS at next step & N/A & N/A & \\
\hline $9-4$ & \multicolumn{7}{|c|}{ Align inert gas three way valve V-609 to STS Cask position } \\
\hline $9-4.1$ & $\begin{array}{l}\text { three way valve } \\
\text { not aligned to } \\
\text { STS }\end{array}$ & Error & None & $\begin{array}{l}\text { operational upset, inerting gas to working area if } \mathrm{V}-610 \text { not } \\
\text { closed; if closed, no flow }\end{array}$ & N/A & $\mathrm{N} / \mathrm{A}$ & $\begin{array}{l}\text { Inerting gas in operating area addressed } \\
\text { in step 9-5 }\end{array}$ \\
\hline $9-5$ & \multicolumn{7}{|c|}{ Open V-608 to Purge STS cask with inert gas. Stop the Cask Purge Clock. } \\
\hline $9-5.1$ & No flow & \begin{tabular}{|l|} 
Valve alignment not \\
accomplished \\
\end{tabular} & None & $\begin{array}{l}\text { Operational upset, STS purge not started, added time against } \\
\text { inerting clock. }\end{array}$ & N/A & N/A & \\
\hline $9-5.2$ & \begin{tabular}{|l|} 
Flow through \\
poor connection \\
or wrong \\
pathway \\
\end{tabular} & Errors in steps 9-3, 9-5, 9-6 & Industrial hazard & Inerting gas to work area, personnel hazard & N/A & N/A & \\
\hline
\end{tabular}


Table D-1 "What/If" HAZARDS ANALYSIS RESULTS- OPERATION SEQUENCES (GAP ANALYSIS)

\begin{tabular}{|c|c|c|c|c|c|c|c|}
\hline \multirow[t]{2}{*}{ Identifier* } & \multirow[t]{2}{*}{ "What/If" } & \multirow[t]{2}{*}{ Causes } & \multirow{2}{*}{$\begin{array}{l}\text { Hazardous } \\
\text { Condition }\end{array}$} & \multirow[t]{2}{*}{ Consequences } & \multicolumn{2}{|c|}{ Candidate Controls } & \multirow[t]{2}{*}{ Remarks/Actions } \\
\hline & & & & & Engineered Features & Admin Controls & \\
\hline $9-6$ & \multicolumn{7}{|c|}{ Secure from STS Cask Inert Gas Purge upon O2 Sensor AI-760- 601 reading less than 1 vol\%: Close V-608. Start the Shipping Window Clock } \\
\hline $9-6.1$ & $\begin{array}{l}\text { Oxygen sensor } \\
\text { not functioning }\end{array}$ & $\begin{array}{l}\text { Faulty instrument or valves not } \\
\text { open or sampling pump not } \\
\text { operation }\end{array}$ & Deflagration & $\begin{array}{l}\text { Operational upset, Not acceptable for transport (TSR) } \\
\text { STS not inert, may have flammable environment in cask, } \\
\text { leading to potential deflagration in cask. }\end{array}$ & None & $\begin{array}{l}\text { Procedures } \\
\text { Training } \\
\text { Conduct of operations }\end{array}$ & \\
\hline $9-6.2$ & $\begin{array}{l}\text { Stop early on } \\
\text { purge }\end{array}$ & error & Deflagration & $\begin{array}{l}\text { Operational upset, Not acceptable for transport (TSR) } \\
\text { STS not inert, may have flammable environment in cask, } \\
\text { leading to potential deflagration in cask }\end{array}$ & Oxygen sensor & $\begin{array}{l}\text { Procedures } \\
\text { Training } \\
\text { Conduct of operations }\end{array}$ & \\
\hline 9-7 & \multicolumn{7}{|c|}{ Close STS vent port } \\
\hline $9-7.1$ & $\begin{array}{l}\text { Step not } \\
\text { performed }\end{array}$ & Error & None & $\begin{array}{l}\text { Operational upset } \\
\text { Cask will not pressurize on next step }\end{array}$ & N/A & N/A & \\
\hline $9-8$ & \multicolumn{7}{|c|}{ Open needle valve V-607 to pressurize the STS with Inert Gas to approximately 3 psi (PI-760-606). Close V-607. } \\
\hline $9-8.1$ & $\begin{array}{l}\text { Too much } \\
\text { pressure }\end{array}$ & Error & overpressure & $\begin{array}{l}\text { Not acceptable for transport (TSR) Shorten shipping window } \\
\text { based on pressure }\end{array}$ & Pressure gauge & \begin{tabular}{|l|} 
Procedures \\
Training \\
Conduct of operations
\end{tabular} & . \\
\hline $9-8.2$ & $\begin{array}{l}\text { Can't measure } \\
\text { pressure }\end{array}$ & V-608 not open & None & Not acceptable for transport (TSR) & N/A & N/A & \\
\hline $9-9$ & \multicolumn{7}{|c|}{ Disconnect vent line from vent port tool } \\
\hline $9-9.1$ & $\begin{array}{l}\text { Contaminated } \\
\text { tool }\end{array}$ & $\begin{array}{l}\text { Connector contaminate when } \\
\text { connected to STSC }\end{array}$ & $\begin{array}{l}\text { Spread of } \\
\text { contamination }\end{array}$ & Operational upset, recovery/decon & None & \begin{tabular}{|l|} 
RadCon Program \\
\end{tabular} & \\
\hline $9-10$ & \multicolumn{7}{|c|}{ Close STS drain port } \\
\hline $9-10.1$ & Doesn't work & Threads fouled & None & $\begin{array}{l}\text { Operational Upset, Rework, delay } \\
\text { Potential shipment delay }\end{array}$ & N/A & N/A & How to know? \\
\hline $9-11$ & \multicolumn{7}{|c|}{ Relieve inert gas line pressure and disconnect inert gas supply line from drain port tool } \\
\hline $9-11.1$ & $\begin{array}{l}\text { Vent the line and } \\
\text { the STS if port } \\
\text { not closed }\end{array}$ & Error in order of operations & None & Operational Upset, Rework, delay, Potential shipment delay & $\mathrm{N} / \mathrm{A}$ & $\mathrm{N} / \mathrm{A}$ & How to know that pressurization is lost? \\
\hline $9-12$ & \multicolumn{7}{|c|}{ ve vent port to } \\
\hline
\end{tabular}


Table D-1 "What/If" HAZARDS ANALYSIS RESULTS- OPERATION SEQUENCES (GAP ANALYSIS)

\begin{tabular}{|c|c|c|c|c|c|c|c|}
\hline \multirow[t]{2}{*}{ Identifier* } & \multirow[t]{2}{*}{ "What/If" } & \multirow[t]{2}{*}{ Causes } & \multirow{2}{*}{$\begin{array}{l}\text { Hazardous } \\
\text { Condition }\end{array}$} & \multirow[t]{2}{*}{ Consequences } & \multicolumn{2}{|c|}{ Candidate Controls } & \multirow[t]{2}{*}{ Remarks/Actions } \\
\hline & & & & & Engineered Features & Admin Controls & \\
\hline $9-12.1$ & Cap not installed & Threads fouled, error & None & $\begin{array}{l}\text { Operational upset. Rework, delay Must be capped for transport } \\
\text { (TSR) }\end{array}$ & N/A & N/A & \\
\hline $9-13$ & \multicolumn{7}{|c|}{ Install/zip up Inner IP-2 STS lid bag } \\
\hline $9-13.1$ & Fail bag & Error, operational event & None & Operational Upset, Rework, delay & N/A & N/A & \\
\hline $9-14$ & \multicolumn{7}{|c|}{ Install/zip up Outer IP-2 STS lid bag } \\
\hline $9-14.1$ & Fail bag & Error, operational event & None & Operational Upset, Rework, delay, & $\mathrm{N} / \mathrm{A}$ & N/A & \\
\hline $9-15$ & \multicolumn{7}{|c|}{ Remove drain port tool and install drain port cap } \\
\hline $9-15.1$ & $\begin{array}{l}\text { Drain port not } \\
\text { capped }\end{array}$ & Threads fouled ,error & None & $\begin{array}{l}\text { Operational upset, must be capped for transport (TSR) Rework, } \\
\text { delay }\end{array}$ & $\mathrm{N} / \mathrm{A}$ & N/A & \\
\hline $9-16$ & \multicolumn{7}{|c|}{ Close/install IP-2 closure on STS drain port } \\
\hline $9-16.1$ & $\begin{array}{l}\text { Failure to } \\
\text { perform }\end{array}$ & Step skipped, error & None & $\begin{array}{l}\text { Operational upset, must be capped for transport (TSR, rework, } \\
\text { delay }\end{array}$ & N/A & N/A & \\
\hline $9-17$ & \multicolumn{7}{|c|}{ Weigh and record final STS loaded weight (WIT-740-401). } \\
\hline $9-17.1$ & Failure to record & Error & None & $\begin{array}{l}\text { Operational upset, Transportation issue - required on manifest } \\
\text { Rework, delay }\end{array}$ & N/A & N/A & \\
\hline $9-18$ & \multicolumn{7}{|c|}{ Complete preparation of shipping manifest } \\
\hline $9-18.1$ & $\begin{array}{l}\text { Failure to } \\
\text { complete }\end{array}$ & Error & None & $\begin{array}{l}\text { Operational upset, Transportation issue, required to transport, } \\
\text { Rework, delay }\end{array}$ & N/A & N/A & \\
\hline $9-19$ & \multicolumn{7}{|c|}{ Open loading bay door } \\
\hline $9-19.1$ & Won't open & Mechanism problem & None & $\begin{array}{l}\text { Operational upset } \\
\text { Cannot connect tractor to trailer, shipping window clock } \\
\text { ticking }\end{array}$ & N/A & N/A & \\
\hline $9-20$ & \multicolumn{7}{|c|}{ Position tractor and connect to STS trailer } \\
\hline
\end{tabular}


Table D-1 "What/If" HAZARDS ANALYSIS RESULTS- OPERATION SEQUENCES (GAP ANALYSIS)

\begin{tabular}{|c|c|c|c|c|c|c|c|}
\hline \multirow[t]{2}{*}{ Identifier* } & \multirow[t]{2}{*}{ "What/ff" } & \multirow[t]{2}{*}{ Causes } & \multirow{2}{*}{$\begin{array}{l}\text { Hazardous } \\
\text { Condition }\end{array}$} & \multirow[t]{2}{*}{ Consequences } & \multicolumn{2}{|c|}{ Candidate Controls } & \multirow[t]{2}{*}{ Remarks/Actions } \\
\hline & & & & & Engineered Features & Admin Controls & \\
\hline $9-20.1$ & $\begin{array}{l}\text { Tractor mis- } \\
\text { positioned, won't } \\
\text { connect }\end{array}$ & Error, impact & None & $\begin{array}{l}\text { Operational upset, Cannot connect tractor to trailer, shipping } \\
\text { window clock ticking }\end{array}$ & N/A & N/A & \\
\hline 9-21 & \multicolumn{7}{|c|}{ Receive and verify shipping manifest } \\
\hline $9-21.1$ & $\begin{array}{l}\text { Manifest not } \\
\text { complete/ correct }\end{array}$ & error & None & $\begin{array}{l}\text { Operational upset } \\
\text { Cannot leave, shipping window clock ticking }\end{array}$ & $\mathrm{N} / \mathrm{A}$ & N/A & \\
\hline 9-22 & \multicolumn{7}{|c|}{ Remove loaded STS trailer from annex loading bay } \\
\hline 9-22.1 & $\begin{array}{l}\text { Damage } \\
\text { structure/trailer }\end{array}$ & Error & None & $\begin{array}{l}\text { Operational Upset, } \\
\text { Cannot transport, shipping window clock ticking }\end{array}$ & N/A & N/A & \\
\hline $9-23$ & \multicolumn{7}{|c|}{ Close loading bay door } \\
\hline 9-23.1 & Won't close & Mechanism failure & None & $\begin{array}{l}\text { Operational upset } \\
\text { Potential delay for setup of next STSC }\end{array}$ & N/A & N/A & \\
\hline & $\mathrm{nar}$ & defined in Appe & & & & & \\
\hline
\end{tabular}


PRC-STP-00473 REV 0

APPENDIX E

FREQUENCY AND CONSEQUENCE LEVEL RESULTS

E-i 
TABLE E-1. FREQUENCY AND CONSEQUENCE LEVEL RESULTS - SPRAY LEAK/ SPLASH AND SPLATTER SCENARIOS

\begin{tabular}{|c|c|c|c|c|c|c|}
\hline \multirow{2}{*}{ Node } & \multirow{2}{*}{ What-If } & \multirow{2}{*}{ Unmitigated Consequence } & \multirow{2}{*}{$\begin{array}{l}\text { Event } \\
\text { Freq }\end{array}$} & \multicolumn{3}{|c|}{ Consequence } \\
\hline & & & & FW & Onsite & Offsite \\
\hline \multicolumn{7}{|l|}{ SEISMIC } \\
\hline S-2 & $\begin{array}{l}\text { Modified Annex structure or crane collapses due to basin } \\
\text { structure failure during sludge retrieval and loading or } \\
\text { overfill recovery }\end{array}$ & \multirow{3}{*}{$\begin{array}{l}\text { Uncontrolled Release of Sludge: Impact on transfer/overfill recovery line in annex leads to loss of primary } \\
\text { containment, then a spray or splash and splatter release of slurry could occur. }\end{array}$} & \multirow{4}{*}{$\mathrm{U}$} & \multirow{4}{*}{$\mathrm{H}$} & \multirow{4}{*}{$\mathrm{H}$} & \multirow{4}{*}{$\mathrm{L}$} \\
\hline S-4 & $\begin{array}{l}\text { Modified Annex Structure or crane collapses during } \\
\text { transfer or overfill recovery }\end{array}$ & & & & & \\
\hline S-5 & $\begin{array}{l}\text { Hoses disconnected at transfer box during transfer or } \\
\text { overfill recovery }\end{array}$ & & & & & \\
\hline S-3 & $\begin{array}{l}\mathrm{KW} \text { Basin super-structure failure during sludge retrieval } \\
\text { and loading or overfill recovery }\end{array}$ & $\begin{array}{l}\text { Uncontrolled Release of Sludge: Impact on transfer/overfill recovery line in KW Basin area leads to loss of primary } \\
\text { containment, then a spray or splash and splatter release of slurry could occur. }\end{array}$ & & & & \\
\hline S-6 & $\begin{array}{l}\text { Modified Annex structure collapses due to basin structure } \\
\text { failure during decant }\end{array}$ & \multirow{3}{*}{$\begin{array}{l}\text { Uncontrolled Release of Sludge: Impact on decant/backwash line in annex leads to loss of primary containment, then a } \\
\text { spray or splash and splatter release of slurry could occur. }\end{array}$} & \multirow{3}{*}{$\mathrm{U}$} & \multirow{3}{*}{$\mathrm{L}$} & \multirow{3}{*}{$\mathrm{L}$} & \multirow{3}{*}{$\mathrm{L}$} \\
\hline S-7 & Basin super-structure collapses during decant & & & & & \\
\hline S-8 & $\begin{array}{l}\text { Hoses disconnected at decant box or sand filter or STSC } \\
\text { during decant or circulation to inject floc }\end{array}$ & & & & & \\
\hline S-09 & $\begin{array}{l}\text { Modified Annex structure collapses due to KW basin } \\
\text { super-structure failure during backwash }\end{array}$ & \multirow{3}{*}{$\begin{array}{l}\text { Uncontrolled Release of Sludge: Impact on decantbackwash line in annex leads to loss of primary containment, then a } \\
\text { spray or splash and splatter release of slurry could occur. }\end{array}$} & \multirow{3}{*}{$\mathrm{U}$} & \multirow{3}{*}{$\mathrm{L}$} & \multirow{3}{*}{$\mathrm{L}$} & \multirow{3}{*}{$\mathrm{L}$} \\
\hline S-10 & Modified Annex structure collapses during backwash & & & & & \\
\hline S-11 & $\begin{array}{l}\text { Hoses disconnected at sand filter or decant box or STSC } \\
\text { during backwash }\end{array}$ & & & & & \\
\hline S-16 & $\begin{array}{l}\text { Damage to nitrogen bottle rack leads to generation of } \\
\text { bottle missile }\end{array}$ & Uncontrolled Release of Sludge: Potential to impact transfer lines, to event comparable to S-4, $5, \& 8$. & $\mathrm{U}$ & $\mathrm{H}$ & H & $\mathrm{L}$ \\
\hline $\mathrm{S}-20$ & Fire initiated by DBE & $\begin{array}{l}\text { Uncontrolled Release of Sludge: Fire event FF-1 is initiated by and taken in combination with seismic event failure. } \\
\text { Fire damages transfer, decant or overfill recovery transfer line (either directly or via structure failure) }\end{array}$ & $\mathrm{U}$ & $\mathrm{H}$ & $\mathrm{H}$ & $\mathrm{L}$ \\
\hline $\mathrm{S}-21$ & $\begin{array}{l}\text { LOEP initiated by DBE (structural damage to electrical } \\
\text { equipment or distribution) }\end{array}$ & $\begin{array}{l}\text { Uncontrolled Release of Sludge: LOEP-1 event below are initiated by and taken in combination with seismic event } \\
\text { failures. Sludge settles in line, or valve closes resulting in blocked line on restart and system overpressure and line } \\
\text { failure }\end{array}$ & $\mathrm{U}$ & $\mathrm{L}$ & $\mathrm{L}$ & $\mathrm{L}$ \\
\hline S-27 & $\begin{array}{l}\text { Seismic event causes Modified Annex structure or } \\
\text { equipment collapse on to transfer, decant or overfill } \\
\text { recovery transfer lines }\end{array}$ & $\begin{array}{l}\text { Uncontrolled Release of Sludge: Transfer line blockage results in high pressure failure and backward flow in lines } \\
\text { interfacing with the transfer line/decant/overfill recovery lines. }\end{array}$ & $\mathrm{U}$ & $\mathrm{H}$ & $\mathrm{H}$ & $\mathrm{L}$ \\
\hline \multicolumn{7}{|l|}{ HIGH WIND } \\
\hline W-2 & $\begin{array}{l}\text { Modified Annex structure or crane collapses due to KW } \\
\text { basin super-structure failure during sludge retrieval and } \\
\text { loading, decant or overfill recovery }\end{array}$ & $\begin{array}{l}\text { Uncontrolled Release of Sludge: Impact on transfer/overfill recovery/decant/backwash line in annex leads to loss of } \\
\text { primary containment, then a spray or splash and splatter release of slurry could occur. }\end{array}$ & $\mathrm{U}$ & $\mathrm{H}$ & $\mathrm{H}$ & $\mathrm{L}$ \\
\hline
\end{tabular}


TABLE E-1. FREQUENCY AND CONSEQUENCE LEVEL RESULTS - SPRAY LEAK/ SPLASH AND SPLATTER SCENARIOS

\begin{tabular}{|c|c|c|c|c|c|c|}
\hline \multirow{2}{*}{ Node } & \multirow{2}{*}{ What-If } & \multirow{2}{*}{ Unmitigated Consequence } & \multirow{6}{*}{$\begin{array}{l}\text { Event } \\
\text { Freq }\end{array}$} & \multicolumn{3}{|c|}{ Consequence } \\
\hline & & & & FW & Onsite & Offsite \\
\hline W-3 & $\begin{array}{l}\text { KW Basin super-structure failure during sludge retrieval } \\
\text { and loading, decant or overfill recovery }\end{array}$ & $\begin{array}{l}\text { Uncontrolled Release of Sludge: Impact on transfer/overfill recovery/decant/backwash line outside annex leads to loss } \\
\text { of primary containment, then a spray or splash and splatter release of slurry could occur. }\end{array}$ & & & & \\
\hline W-4 & $\begin{array}{l}\text { Missile impact to sludge piping external to the Modified } \\
\text { Annex during loading, decant or overfill recovery }\end{array}$ & \multirow{3}{*}{$\begin{array}{l}\text { Uncontrolled Release of Sludge: Impact on transfer/overfill recovery/decant/backwash line in annex leads to loss of } \\
\text { primary containment, then a spray or splash and splatter release of slurry could occur. }\end{array}$} & & & & \\
\hline W-5 & $\begin{array}{l}\text { Modified Annex Structure collapses during transfer, } \\
\text { decant or overfill recovery }\end{array}$ & & & & & \\
\hline W-6 & $\begin{array}{l}\text { Hoses impacted or disconnected at transfer box or sand } \\
\text { filter or decant box or STSC during transfer, decant, } \\
\text { backwash, overfill recovery }\end{array}$ & & & & & \\
\hline W-8 & Nitrogen bottle overturns, knocks off valve & $\begin{array}{l}\text { Uncontrolled Release of Sludge: Impact on transfer/overfill recovery/decantbackwash line in annex from gas bottle } \\
\text { missile leads to loss of primary containment, then a spray or splash and splatter release of slurry. }\end{array}$ & $\mathrm{U}$ & $\mathrm{H}$ & $\mathrm{H}$ & $\mathrm{L}$ \\
\hline W-19 & $\begin{array}{l}\text { Wind load damages Modified Annex structure leading to } \\
\text { collapse onto transfer lines, decant, backwash, overfill } \\
\text { recovery }\end{array}$ & $\begin{array}{l}\text { Uncontrolled Release of Sludge: Impact on transfer/overfill recovery/decant/backwash line in annex leads to loss of } \\
\text { primary containment, then a spray or splash and splatter release of slurry could occur. }\end{array}$ & $\mathrm{U}$ & $\mathrm{H}$ & $\mathrm{H}$ & $\mathrm{L}$ \\
\hline \multicolumn{7}{|l|}{ SNOW } \\
\hline SF-1 & $\begin{array}{l}\text { Snow load fails Modified Annex structure during transfer, } \\
\text { decant or overfill recovery }\end{array}$ & $\begin{array}{l}\text { Uncontrolled Release of Sludge: Impact on transfer//verfill recovery/decant/backwash line in annex leads to loss of } \\
\text { primary containment, then a spray or splash and splatter release of slurry could occur. }\end{array}$ & $\mathrm{U}$ & $\mathrm{H}$ & $\mathrm{H}$ & $\mathrm{L}$ \\
\hline SF-9 & $\begin{array}{l}\text { Snowfall fails Modified Annex structure and impacts } \\
\text { transfer lines }\end{array}$ & $\begin{array}{l}\text { Uncontrolled Release of Sludge: Transfer lines are impacted by a structure or equipment collapse, dead heading the } \\
\text { transfer or decant or overfill recovery, and over pressuring an interfacing service system pipe with resulting spray or } \\
\text { splash and splatter }\end{array}$ & $\mathrm{U}$ & $\mathrm{H}$ & $\mathbf{H}$ & $\mathrm{L}$ \\
\hline \multicolumn{7}{|l|}{ ASHFALL } \\
\hline AF-1 & $\begin{array}{l}\text { Ashfall load fails Modified Annex structure during } \\
\text { transfer, decant or overfill recovery }\end{array}$ & $\begin{array}{l}\text { Uncontrolled Release of Sludge: Impact on transfer/overfill recovery/decant/backwash line in annex leads to loss of } \\
\text { primary containment, then a spray or splash and splatter release of slurry could occur. }\end{array}$ & $\mathrm{U}$ & $\mathrm{H}$ & $\mathrm{H}$ & $\mathrm{L}$ \\
\hline AF-9 & $\begin{array}{l}\text { Ashfall fails Modified Annex structure and impacts } \\
\text { transfer lines }\end{array}$ & $\begin{array}{l}\text { Uncontrolled Release of Sludge: Transfer lines are impacted by a structure or equipment collapse, dead heading the } \\
\text { transfer or decant or overfill recovery, and over pressuring an interfacing service system pipe with resulting spray or } \\
\text { splash and splatter }\end{array}$ & $\mathrm{U}$ & $\mathbf{H}$ & $\mathbf{H}$ & $\mathrm{L}$ \\
\hline \multicolumn{7}{|c|}{ LIGHTNING } \\
\hline L-5 & Lightning damages interlocks or controls & Uncontrolled Release of Sludge: Lightning strike makes interlocks for process inoperable leading to overfill and leak & $\mathrm{U}$ & $\mathrm{L}$ & $\mathrm{L}$ & L. \\
\hline \multicolumn{7}{|c|}{ LOW AMBIENT TEMPERATURE } \\
\hline LAT-5 & $\begin{array}{l}\text { Fire initiates during freezing condition which had frozen } \\
\text { Modified Annex sprinkler system, }\end{array}$ & $\begin{array}{l}\text { Uncontrolled release of sludge: Loss of safety SSC, potential for unmitigated fire. Unmitigated fire leads to loss of } \\
\text { ventilation or leak. }\end{array}$ & $\mathrm{U}$ & $\mathrm{H}$ & $\mathrm{H}$ & $\mathrm{L}$ \\
\hline
\end{tabular}


TABLE E-1. FREQUENCY AND CONSEQUENCE LEVEL RESULTS - SPRAY LEAK/ SPLASH AND SPLATTER SCENARIOS

\begin{tabular}{|c|c|c|c|c|c|c|}
\hline \multirow{2}{*}{ Node } & \multirow{2}{*}{ What-If } & \multirow{2}{*}{ Unmitigated Consequence } & \multirow{2}{*}{$\begin{array}{l}\text { Event } \\
\text { Freq }\end{array}$} & \multicolumn{3}{|c|}{ Consequence } \\
\hline & & & & FW & Onsite & Offsite \\
\hline \multicolumn{7}{|c|}{ LOSS OF ELECTRICAL POWER } \\
\hline LOEP-1 & Loss of power during transfer or overfill recovery & $\begin{array}{l}\text { Uncontrolled Release of Sludge: Sludge settles in line, or valve closes resulting in blocked line on restart and system } \\
\text { overpressure with attendant overpressure with resulting spray or splash and splatter }\end{array}$ & $\mathrm{U}$ & $\mathbf{H}$ & $\mathrm{H}$ & $\mathrm{L}$ \\
\hline \multicolumn{7}{|c|}{ VEHICLE IMPACT } \\
\hline VI-2 & $\begin{array}{l}\text { Vehicle impacts Modified Annex structure while loading } \\
\text { STSC or overfill recovery }\end{array}$ & $\begin{array}{l}\text { Uncontrolled Release of Sludge: Vehicle impact to structure results in transfer line failure resulting in a spray or splash } \\
\text { and splatter release of slurry }\end{array}$ & $\mathrm{U}$ & $\mathrm{H}$ & $\mathrm{H}$ & $\mathrm{L}$ \\
\hline \multicolumn{7}{|c|}{ AIR CRAFT CRASH } \\
\hline $\mathrm{ACC}-1$ & Aircraft impacts Modified Annex & $\begin{array}{l}\text { Uncontrolled Release of Sludge: Impact on transfer/overfill recovery/decant/backwash line in annex leads to loss of } \\
\text { primary containment, then a spray or splash and splatter release of slurry could occur. }\end{array}$ & BEU & $\mathrm{H}$ & $\mathrm{H}$ & $\mathrm{L}$ \\
\hline \multicolumn{7}{|c|}{ EXTERNAL EXPLOSION } \\
\hline $\mathrm{EE}-4$ & $\begin{array}{l}\text { Damage to Modified Annex structure, impacting transfer } \\
\text { lines in service }\end{array}$ & Uncontrolled Release of Sludge: Blocked or failed transfer lines result in a spray or splash and splatter release of slurry & $\mathrm{U}$ & $\mathrm{H}$ & $\mathrm{H}$ & $\mathrm{L}$ \\
\hline \multicolumn{7}{|c|}{ FACILITY FIRE } \\
\hline FF-1 & Fire leads to transfer hose damage & $\begin{array}{l}\text { Uncontrolled Release of Sludge: Fire damages transfer, decant or overfill recovery transfer line (either directly or via } \\
\text { structure failure) leads to a spray or splash and splatter release of slurry }\end{array}$ & $\mathrm{U}$ & $\mathrm{H}$ & $\mathrm{H}$ & $\mathrm{L}$ \\
\hline
\end{tabular}


Table E-2. FREQUENCY AND CONSEQUENCE LEVEL RESULTS -HYDROGEN DEFLAGRATION

\begin{tabular}{|c|c|c|c|c|c|c|}
\hline \multirow{2}{*}{ Node } & \multirow{2}{*}{ What-If } & \multirow{2}{*}{ Unmitigated Consequence } & \multirow{2}{*}{$\begin{array}{l}\text { Event } \\
\text { Freq }\end{array}$} & \multicolumn{3}{|c|}{ Consequence } \\
\hline & & & & FW & Onsite & Offsite \\
\hline \multicolumn{7}{|c|}{ SEISMIC } \\
\hline S-12 & $\begin{array}{l}\text { Modified Annex Structure collapses due to KW basin } \\
\text { super-structure failure after sludge retrieval and loading }\end{array}$ & \multirow{4}{*}{$\begin{array}{l}\text { Hydrogen Deflagration: Impact to ventilation hoses, ducting, stack, or ventilation equipment shuts down STSC } \\
\text { headspace purge, resulting in buildup of hydrogen with potential for deflagration. }\end{array}$} & \multirow{4}{*}{$\mathrm{U}$} & \multirow{4}{*}{$\mathrm{H}$} & \multirow{4}{*}{$\mathrm{L}$} & \multirow{4}{*}{$\mathrm{L}$} \\
\hline S-13 & $\begin{array}{l}\text { Modified Annex Structure collapse or crane drop after } \\
\text { sludge retrieval and loading }\end{array}$ & & & & & \\
\hline S-14 & $\begin{array}{l}\text { Modified Annex Structure collapse or crane drop or cask } \\
\text { lid drop during installation }\end{array}$ & & & & & \\
\hline S-16 & $\begin{array}{l}\text { Damage to nitrogen bottle rack leads to generation of } \\
\text { bottle missile }\end{array}$ & & & & & \\
\hline S-26 & $\begin{array}{l}\text { Loss of normal and backup power (structural damage to } \\
\text { backup electrical equipment) }\end{array}$ & $\begin{array}{l}\text { Hydrogen Deflagration: Common cause LOEP and loss of backup power, leading to STSC heat up, hydrogen buildup } \\
\text { with potential for deflagration. }\end{array}$ & $\mathrm{U}$ & $\mathrm{H}$ & $\mathrm{L}$ & $\mathrm{L}$ \\
\hline \multicolumn{7}{|c|}{ HIGH WIND } \\
\hline W-9 & $\begin{array}{l}\text { Modified Annex Ventilation equipment damaged and/or } \\
\text { inoperable }\end{array}$ & Hydrogen Deflagration: Nitrogen bottle overturns, knocks off valve with loss of purge and hydrogen buildup & $\mathrm{U}$ & H & $\mathrm{L}$ & $\mathrm{L}$ \\
\hline W-10 & $\begin{array}{l}\text { Modified Annex Ventilation equipment damaged and/or } \\
\text { inoperable }\end{array}$ & $\begin{array}{l}\text { Hydrogen Deflagration: Impact to duct manifold, stack collapse, impact to power or control panels, on site LOEP lead } \\
\text { to loss of STSC purge and hydrogen buildup }\end{array}$ & $\mathrm{U}$ & $\mathrm{H}$ & $\mathrm{L}$ & $\mathrm{L}$ \\
\hline W-11 & $\begin{array}{l}\text { Modified Annex Ventilation filters become inoperable due } \\
\text { to dust }\end{array}$ & $\begin{array}{l}\text { Hydrogen Deflagration: Dust loading plugs inlet and or out filters leading to ventilation inoperability, loss of purge and } \\
\text { hydrogen buildup }\end{array}$ & $\mathrm{U}$ & $\mathrm{H}$ & $\mathrm{L}$ & $\mathrm{L}$ \\
\hline W-15 & Wind or missile damage leads to fire & Hydrogen Deflagration: Fire incapacitates the ventilation, leading to hydrogen accumulation & $\mathrm{U}$ & $\mathrm{H}$ & $\mathrm{L}$ & $\mathrm{L}$ \\
\hline \multicolumn{7}{|c|}{ FLOOD } \\
\hline FL-3 & Flood damages ventilation & $\begin{array}{l}\text { Hydrogen Deflagration: Accidental sprinkler actuation impacts ventilation controls incapacitating system operability } \\
\text { with resulting loss of purge, hydrogen accumulation }\end{array}$ & $\mathrm{U}$ & н & $\mathrm{L}$ & $\mathrm{L}$ \\
\hline \multicolumn{7}{|c|}{ sNow } \\
\hline SF-2 & Snow load fails Modified Annex structure & Hydrogen Deflagration: Structure failure impacts ventilation and prevents STSC purge leading to hydrogen buildup & $\mathrm{U}$ & $\mathrm{H}$ & $\mathrm{L}$ & $\mathrm{L}$ \\
\hline SF-12 & $\begin{array}{l}\text { Modified Annex Roof failure or Ice dam and water } \\
\text { inleakge }\end{array}$ & $\begin{array}{l}\text { Hydrogen Deflagration: Water inleakge impacts HVAC Controls and prevents STSC purge leading to hydrogen } \\
\text { buildup }\end{array}$ & $\mathrm{U}$ & $\mathrm{H}$ & $\mathrm{L}$ & $\mathrm{L}$ \\
\hline \multicolumn{7}{|c|}{ ASHFALL } \\
\hline $\mathrm{AF}-2$ & Ashfall load fails Modified Annex structure & Hydrogen Deflagration: Structure failure impacts ventilation and prevents STSC purge leading to hydrogen buildup & $\mathrm{U}$ & $\mathrm{H}$ & $\mathrm{L}$ & $\mathrm{L}$ \\
\hline
\end{tabular}


Table E-2. FREQUENCY AND CONSEQUENCE LEVEL RESULTS -HYDROGEN DEFLAGRATION

\begin{tabular}{|c|c|c|c|c|c|c|}
\hline \multirow{2}{*}{ Node } & \multirow{2}{*}{ What-If } & \multirow{2}{*}{ Unmitigated Consequence } & \multirow{2}{*}{$\begin{array}{l}\text { Event } \\
\text { Freq }\end{array}$} & \multicolumn{3}{|c|}{ Consequence } \\
\hline & & & & Fw & Onsite & Offsite \\
\hline AF-12 & $\begin{array}{l}\text { Modified Annex Ventilation filters become inoperable due } \\
\text { to ash }\end{array}$ & Hydrogen Deflagration: & $\mathrm{U}$ & $\mathrm{H}$ & $\mathrm{L}$ & $\mathrm{L}$ \\
\hline \multicolumn{7}{|l|}{ RAIN } \\
\hline R-2 & $\begin{array}{l}\text { Heavy rainfall and winds result in wetting of electrical } \\
\text { equipment }\end{array}$ & $\begin{array}{l}\text { Hydrogen Deflagration: Wet electrical components cause failure ventilation system, leading to buildup of hydrogen in } \\
\text { STSC and Uncontrolled release of sludge, worker and public dose. }\end{array}$ & $\mathrm{U}$ & $\mathrm{H}$ & $\mathrm{L}$ & $\mathrm{L}$ \\
\hline \multicolumn{7}{|c|}{ LIGHTNING } \\
\hline $\mathrm{L}-2$ & $\begin{array}{l}\text { Lightning damages Modified Annex HVAC switchgear or } \\
\text { control system }\end{array}$ & $\begin{array}{l}\text { Hydrogen Deflagration: Loss of ventilation flow to loaded STSC leads to hydrogen buildup, and Uncontrolled release } \\
\text { of sludge, worker and public dose. }\end{array}$ & $\mathrm{U}$ & H & $\mathrm{L}$ & $\mathrm{L}$ \\
\hline \multicolumn{7}{|c|}{ LOW AMBIENT TEMPERATURE } \\
\hline LAT-4 & Freezing condition impacts STSC vent & $\begin{array}{l}\text { Hydrogen Deflagration: Moisture separator freezes preventing gas flow. Buildup of hydrogen leading to Uncontrolled } \\
\text { release of sludge, worker and public dose. }\end{array}$ & $\mathrm{U}$ & $\mathrm{H}$ & $\mathrm{L}$ & $\mathrm{L}$ \\
\hline LAT-5 & $\begin{array}{l}\text { Fire initiates when freezing condition freezes Modified } \\
\text { Annex sprinkler system }\end{array}$ & $\begin{array}{l}\text { Hydrogen Deflagration: Loss of safety SSC, potential for unmitigated fire. Unmitigated fire leads to loss of ventilation } \\
\text { and Potential for Uncontrolled release of sludge, worker and public dose. }\end{array}$ & $\mathrm{U}$ & $\mathrm{H}$ & $\mathrm{L}$ & $\mathrm{L}$ \\
\hline \multicolumn{7}{|c|}{ LOSS OF ELECTRICAL POWER } \\
\hline LOEP-2 & Loss of power to ventilation system when STSC is loaded & Hydrogen Deflagration: Accumulation of hydrogen leading to uncontrolled release of sludge, worker and public dose. & $\mathrm{U}$ & $\mathrm{H}$ & $\mathrm{L}$ & $\mathrm{L}$ \\
\hline LOEP-4 & Loss of power when closing STS & $\begin{array}{l}\text { Hydrogen Deflagration: Stuck or dropped STS lid causes inability to purge or ventilate leading to hydrogen } \\
\text { accumulation and uncontrolled release of sludge, worker and public dose. }\end{array}$ & $\mathrm{U}$ & $\mathrm{H}$ & $\mathrm{L}$ & $\mathrm{L}$ \\
\hline \multicolumn{7}{|c|}{ RANGE FIRE } \\
\hline $\mathrm{RF}-4$ & Modified Annex Structure failure & $\begin{array}{l}\text { Hydrogen Deflagration: Range fire fails structure and prevents purge of STSC or overturns STSC leading to } \\
\text { Uncontrolled release of sludge, worker and public dose. }\end{array}$ & $\mathrm{U}$ & $\mathrm{H}$ & $\mathrm{L}$ & $\mathrm{L}$ \\
\hline $\mathrm{RF}-6$ & Fire smoke and soot plugs HVAC filters & $\begin{array}{l}\text { Hydrogen Deflagration: Smoke and soot plugs HVAC, preventing ventilation of the STSC, buildup of hydrogen and } \\
\text { Uncontrolled release of sludge, worker and public dose. }\end{array}$ & $\mathrm{U}$ & $\mathrm{H}$ & $\mathrm{L}$ & $\mathrm{L}$ \\
\hline \multicolumn{7}{|c|}{ VEHICLE IMPACT } \\
\hline VI-7 & Vehicle impacts bottle storage & $\begin{array}{l}\text { Hydrogen Deflagration: Loss of inerting capability, potential bottle missile damage to ventilation capability and } \\
\text { hydrogen buildup to potential }\end{array}$ & $\mathrm{U}$ & $\mathrm{H}$ & $\mathrm{L}$ & $\mathrm{L}$ \\
\hline \multicolumn{7}{|c|}{ AIR CRAFT CRASH } \\
\hline ACC-1 & Aircraft impacts Modified Annex & Hydrogen Deflagration: Impact on ventilation system, then a hydrogen build up. & $\mathrm{BEU}$ & $\mathrm{H}$ & $\mathrm{H}$ & L \\
\hline
\end{tabular}


Table E-2. FREQUENCY AND CONSEQUENCE LEVEL RESULTS -HYDROGEN DEFLAGRATION

\begin{tabular}{|c|c|c|c|c|c|c|}
\hline \multirow{2}{*}{ Node } & \multirow{2}{*}{ What-If } & \multirow{2}{*}{ Unmitigated Consequence } & \multirow{2}{*}{$\begin{array}{l}\text { Event } \\
\text { Freq }\end{array}$} & \multicolumn{3}{|c|}{ Consequence } \\
\hline & & & & FW & Onsite & Offsite \\
\hline \multicolumn{7}{|c|}{ EXTERNAL FACILITY FIRE } \\
\hline EFF-2 & $\begin{array}{l}\text { Initiates KW Basin super-structure collapse against } \\
\text { modified Annex }\end{array}$ & $\begin{array}{l}\text { Hydrogen Deflagration: Impacted structure prevents ventilation of STSC or departure of loaded STS, hydrogen } \\
\text { accumulation }\end{array}$ & $\mathrm{U}$ & $\mathrm{H}$ & $\mathrm{L}$ & $\mathrm{L}$ \\
\hline EFF-5 & Smoke plugs ventilation & $\begin{array}{l}\text { Hydrogen Deflagration: Impacted structure prevents ventilation of STSC or departure of loaded STS, hydrogen } \\
\text { accumulation }\end{array}$ & $\mathrm{U}$ & $\mathrm{H}$ & $\mathrm{L}$ & $\mathrm{L}$ \\
\hline \multicolumn{7}{|c|}{ FACILITY FIRE } \\
\hline FF-2 & $\begin{array}{l}\text { Fire causes loss of Modified Annex ventilation fans or } \\
\text { plugging filters }\end{array}$ & Hydrogen Deflagration: Loss of vessel ventilation allows hydrogen accumulation & $\mathrm{U}$ & $\mathrm{H}$ & $\mathrm{L}$ & $\mathrm{L}$ \\
\hline FF-3 & Modified Annex Facility fire impacts loaded STSC or STS & $\begin{array}{l}\text { Hydrogen Deflagration: Fire heats STSC, fails hoses, or heats loaded STS. Leads to loss of ventilation for } \\
\text { STSC/deflagration }\end{array}$ & $\mathrm{U}$ & $\mathrm{H}$ & $\mathrm{L}$ & $\mathrm{L}$ \\
\hline
\end{tabular}


Table E-3 FREQUENCY AND CONSEQUENCE LEVEL RESULTS -OVERPRESSURE

\begin{tabular}{|c|c|c|c|c|c|c|}
\hline \multirow{2}{*}{ Node } & \multirow{2}{*}{ What-If } & \multirow{2}{*}{ Unmitigated Consequence } & \multirow{2}{*}{$\begin{array}{l}\text { Event } \\
\text { Freq }\end{array}$} & \multicolumn{3}{|c|}{ Consequence } \\
\hline & & & & FW & Onsite & Offsite \\
\hline \multicolumn{7}{|c|}{ SEISMIC } \\
\hline S-15 & $\begin{array}{l}\text { Modified Annex Structure damage, trailer damage or door } \\
\text { damage preventing load departure }\end{array}$ & $\begin{array}{l}\text { Overpressurization: STS load heats up, hydrogen gas is generated leading to uncontrolled release of sludge, worker } \\
\text { and public dose. }\end{array}$ & $\mathrm{U}$ & $\mathrm{L}$ & $\mathrm{L}$ & $\mathrm{L}$ \\
\hline S-17 & $\begin{array}{l}\text { Damage to T Plant or travel route significantly delays } \\
\text { transfer }\end{array}$ & $\begin{array}{l}\text { Overpressurization: STS load heats up, hydrogen gas is generated leading to uncontrolled release of sludge, worker } \\
\text { and public dose. }\end{array}$ & $\mathrm{U}$ & $\mathrm{L}$ & $\mathrm{L}$ & $\mathrm{L}$ \\
\hline $\mathrm{s}-20$ & Fire initiated by DBE & $\begin{array}{l}\text { Overpressurization: Fire events FF-1 through FF- } 6 \text { below are initiated by and taken in combination with seismic event } \\
\text { failures uncontrolled release of sludge, worker and public dose. }\end{array}$ & $\mathrm{U}$ & $\mathrm{L}$ & $\mathrm{L}$ & $\mathrm{L}$ \\
\hline S-21 & $\begin{array}{l}\text { LOEP initiated by DBE (structural damage to electrical } \\
\text { equipment or distribution) }\end{array}$ & $\begin{array}{l}\text { Overpressurization: LOEP-1 through LOEP- } 5 \text { events below are initiated by and taken in combination with seismic } \\
\text { event failures. Uncontrolled release of sludge, worker and public dose. (See LOEP events below for failure } \\
\text { configurations) }\end{array}$ & $\mathrm{U}$ & $\mathrm{L}$ & $\mathrm{L}$ & $\mathrm{L}$ \\
\hline \multicolumn{7}{|c|}{ HIGH WIND } \\
\hline w-8 & Wind damage to Modified Annex structure or door & $\begin{array}{l}\text { Overpressurization: loaded STS delayed, resulting in heat up and hydrogen generation leading to Uncontrolled release } \\
\text { of sludge, worker and public dose. }\end{array}$ & $\mathrm{U}$ & $\mathrm{L}$ & $\mathrm{L}$ & $\mathrm{L}$ \\
\hline W-14 & Modified Annex structure collapse, fire & $\begin{array}{l}\text { Overpressurization: Fire initiated by some modified annex structural failure in high wind results in common cause } \\
\text { failure of fire suppression system, fire involves loaded STSC or STS, Uncontrolled release of sludge, worker and } \\
\text { public dose. }\end{array}$ & $\mathrm{U}$ & $\mathrm{L}$ & $\mathrm{L}$ & $\mathrm{L}$ \\
\hline \multicolumn{7}{|c|}{ FLOOD } \\
\hline FL-1 & Flood damage prevents STS Transport & $\begin{array}{l}\text { Overpressurization: Water line failure floods/undermines road and prevents STS transport leading to Uncontrolled } \\
\text { release of sludge, worker and public dose. }\end{array}$ & $\mathrm{U}$ & $\mathrm{L}$ & $\mathrm{L}$ & $\mathrm{L}$ \\
\hline \multicolumn{7}{|c|}{ SNOw } \\
\hline SF-3 & Snow load fails Modified Annex structure & Overpressurization: Structure failure prevents loaded STS from departing resulting in hydrogen gas generation, & $\mathrm{U}$ & $\mathrm{L}$ & $\mathrm{L}$ & $\mathrm{L}$ \\
\hline SF-6 & Snowfall prevents transport to T Plant & Overpressurization: Road closure/transportation restriction prevent transport of STS, leading to build up of hydrogen & $\mathrm{U}$ & $\mathrm{L}$ & L & $\mathrm{L}$ \\
\hline
\end{tabular}


Table E-3 FREQUENCY AND CONSEQUENCE LEVEL RESULTS -OVERPRESSURE

\begin{tabular}{|c|c|c|c|c|c|c|}
\hline \multirow{2}{*}{ Node } & \multirow{2}{*}{ What-If } & \multirow{2}{*}{ Unmitigated Consequence } & \multirow{2}{*}{ Event } & \multicolumn{3}{|c|}{ Consequence } \\
\hline & & & & FW & Onsite & Offsite \\
\hline \multicolumn{7}{|c|}{ ASHFALL } \\
\hline AF-3 & Ashfall load fails Modified Annex structure & Overpressurization: Structure failure prevents loaded STS from departing resulting in hydrogen gas generation, & $\mathrm{U}$ & $\mathrm{L}$ & $\mathrm{L}$ & $\mathrm{L}$ \\
\hline AF-6 & Ashfall prevents transport to $\mathrm{T}$ Plant & Overpressurization: Road closure/transportation restriction prevent transport of STS, leading to build up of hydrogen & $\mathrm{U}$ & $\mathrm{L}$ & $\mathrm{L}$ & $\mathrm{L}$ \\
\hline \multicolumn{7}{|l|}{ RAIN } \\
\hline R-1 & Heavy rainfall delays transport & Overpressurization: Delay in transportation of sealed cask leads to heat up and hydrogen pressurization of STS & $\mathrm{U}$ & $\mathrm{L}$ & $\mathrm{L}$ & $\mathrm{L}$ \\
\hline \multicolumn{7}{|c|}{ LIGHTNING } \\
\hline L-4 & Transport of loaded STS delayed by lightning & Overpressurization: Delay in transportation of sealed cask leads to heat up and hydrogen pressurization of STS & $\mathrm{U}$ & $\mathrm{L}$ & $\mathrm{L}$ & $\mathrm{L}$ \\
\hline \multicolumn{7}{|c|}{ HIGH AMBIENT TEMPERATURE } \\
\hline HAT-1 & Heat impacts shipping window & Overpressurization: Delay in transportation of sealed cask leads to heat up and hydrogen pressurization of STS & $\mathrm{U}$ & $\mathrm{L}$ & $\mathrm{L}$ & $\mathrm{L}$ \\
\hline \multicolumn{7}{|c|}{ LOW AMBIENT TEMPERATURE } \\
\hline LAT-1 & Freezing conditions impact shipping window & Overpressurization: Delay in transportation of sealed cask leads to heat up and hydrogen pressurization of STS & $\mathrm{U}$ & $\mathrm{L}$ & $\mathrm{L}$ & $\mathrm{L}$ \\
\hline \multicolumn{7}{|c|}{ LOSS OF ELECTRICAL POWER } \\
\hline LOEP-3 & Loss of power when STS loaded. & Overpressurization: Closed roll up door prevents departure, leading to gas accumulation & $\mathrm{U}$ & $\mathrm{L}$ & $\mathrm{L}$ & $\mathrm{L}$ \\
\hline \multicolumn{7}{|c|}{ RANGE FIRE } \\
\hline RF-1 & Delays departure of loaded STS & Overpressurization: Range fire prevents departure, leading to gas accumulation & $\mathrm{U}$ & $\mathrm{L}$ & $\mathrm{L}$ & $\mathrm{L}$ \\
\hline \multicolumn{7}{|c|}{ VEHICLE IMPACT } \\
\hline VI-3 & $\begin{array}{l}\text { Vehicle Impacts Modified Annex structure with loaded } \\
\text { STS inside }\end{array}$ & Overpressurization: Vehicle impact prevents loaded STS transport with gas accumulation & $\mathrm{U}$ & $\mathrm{L}$ & $\mathrm{L}$ & $\mathrm{L}$ \\
\hline VI-6 & Tractor impacts fuel tank during prep for departure & Overpressurization: Vehicle fuel fire and tire fire impacts STS heating & $\mathrm{u}$ & $\mathrm{L}$ & $\mathrm{L}$ & $\mathrm{L}$ \\
\hline
\end{tabular}


Table E-3 FREQUENCY AND CONSEQUENCE LEVEL RESULTS -OVERPRESSURE

\begin{tabular}{|c|c|c|c|c|c|c|}
\hline \multirow{2}{*}{ Node } & \multirow{2}{*}{ What-If } & \multirow{2}{*}{ Unmitigated Consequence } & \multirow{2}{*}{$\begin{array}{l}\text { Event } \\
\text { Freq }\end{array}$} & \multicolumn{3}{|c|}{ Consequence } \\
\hline & & & & FW & Onsite & Offsite \\
\hline \multicolumn{7}{|c|}{ AIR CRAFT CRASH } \\
\hline ACC-1 & Aircraft impacts Modified Annex & Overpressure: Impact on STS in annex leads excess time in closed vessel and build up of pressure.. & BEU & $\mathrm{H}$ & $\mathrm{H}$ & $\mathrm{L}$ \\
\hline \multicolumn{7}{|c|}{ EXTERNAL EXPLOSION } \\
\hline EE-1 & *Damage to Modified Annex during departure prep & Overpressurization: Damage prevents STS departure allowing gas accumulation & $\mathrm{u}$ & $\mathrm{L}$ & $\mathrm{L}$ & $\mathrm{L}$ \\
\hline \multicolumn{7}{|c|}{ EXTERNAL FACILITY FIRE } \\
\hline EFF-2 & $\begin{array}{l}\text { Initiates KW Basin super-structure collapse against } \\
\text { modified Annex }\end{array}$ & $\begin{array}{l}\text { Overpressurization: Impacted structure prevents ventilation of STSC or departure of loaded STS, hydrogen } \\
\text { accumulation }\end{array}$ & $\mathrm{U}$ & $\mathrm{L}$ & $\mathrm{L}$ & $\mathrm{L}$ \\
\hline \multicolumn{7}{|c|}{ FACILITY FIRE } \\
\hline FF-3 & Modified Annex Facility fire impacts loaded STSC or STS & $\begin{array}{l}\text { Overpressurization: Fire heats STSC, fails hoses, or heats loaded STS. Leads to loss of ventilation with potentially an } \\
\text { overpressure }\end{array}$ & $\mathrm{U}$ & $\mathrm{L}$ & $\mathrm{L}$ & $\mathrm{L}$ \\
\hline FF-6 & Loaded STS not able to leave & Overpressurization: Fire damage prevents hauling loaded STS, resulting in gas accumulation & $\mathrm{U}$ & $\mathrm{L}$ & $\mathrm{L}$ & L \\
\hline
\end{tabular}


Table E-4 FREQUENCY AND CONSEQUENCE LEVEL RESULTS -OVERTURN/SPILL SCENARIOS

\begin{tabular}{|c|c|c|c|c|c|c|}
\hline \multirow{2}{*}{ Node } & \multirow{2}{*}{ What-If } & \multirow{2}{*}{ Unmitigated Consequence } & \multirow{2}{*}{$\begin{array}{c}\text { Event } \\
\text { Freq }\end{array}$} & \multicolumn{3}{|c|}{ Consequence } \\
\hline & & & & FW & Onsite & Offsite \\
\hline \multicolumn{7}{|l|}{ SEISMIC } \\
\hline S-19 & STSC overturns after loading & STSC Overturn Spill: Spill of sludge into loading bay & $\mathrm{U}$ & $\mathrm{L}$ & $\mathrm{L}$ & $\mathrm{L}$ \\
\hline \multicolumn{7}{|l|}{ HIGH WIND } \\
\hline $\mathrm{W}-7$ & Modified Annex Structure failure onto loaded STSC & STSC Overturn Spill: Spill of sludge into loading bay & $\mathrm{U}$ & $\mathrm{L}$ & $\mathrm{L}$ & $\mathrm{L}$ \\
\hline \multicolumn{7}{|l|}{ LIGHTNING } \\
\hline L-5 & Lightning damages interlocks or controls & $\begin{array}{l}\text { STSC Overfill Spill : Lightning strike makes interlocks for process inoperable leading to overfill and spill of sludge into } \\
\text { loading bay }\end{array}$ & $\mathrm{U}$ & $\mathrm{L}$ & $\mathrm{L}$ & $\mathrm{L}$ \\
\hline \multicolumn{7}{|c|}{ RANGE FIRE } \\
\hline $\mathrm{RF}-4$ & Modified Annex Structure failure & STSC Overturn Spill: Spill of sludge into loading bay & $\mathrm{U}$ & $\mathrm{L}$ & $\mathrm{L}$ & $\mathrm{L}$ \\
\hline \multicolumn{7}{|c|}{ FACILITY FIRE } \\
\hline FF-4 & Modified Annex Facility fire impacts structure & STSC Overturn Spill: Spill of sludge into loading bay & $\mathrm{u}$ & $\mathrm{L}$ & $\mathrm{L}$ & $\mathrm{L}$ \\
\hline
\end{tabular}




\begin{tabular}{|c|c|c|c|c|c|c|}
\hline \multirow[b]{2}{*}{ Identifier* } & \multirow{2}{*}{ What-If } & \multirow{2}{*}{ Unmitigated Consequence } & \multirow{2}{*}{$\begin{array}{l}\text { Event } \\
\text { Freq }\end{array}$} & \multicolumn{3}{|c|}{ Consequence } \\
\hline & & & & FW & Onsite & Offsite \\
\hline \multicolumn{7}{|c|}{ Mode 1 PREPARE STSC } \\
\hline $1-17$ & Remove the STS Lid and install onto the Lid Inspection Station. & & & & & \\
\hline $1-17.4$ & Wrong STSC type (annulus versus not) & $\begin{array}{l}\text { Cask overpressure. Potential to load material in wrong STSC type with future thermal transient, cask overpressure if } \\
\text { not detected, or return to T Plant to get proper STSC. }\end{array}$ & A & L & $\mathrm{L}$ & $\mathrm{L}$ \\
\hline $1-33$ & \multicolumn{6}{|c|}{ Select Sludge Type on PNL-401 (ECRT-KS-401 to 'K-East', 'KWest', or 'Settler' position) and reset PNL-401: Press HS-402 'reset' pushbutton. } \\
\hline $1-33.1$ & Wrong selection & $\begin{array}{l}\text { Overpressurization: Wrong calculations made in automatic system, potential to overload STSC, resulting in lower } \\
\text { transport window and lesser thermal stability. Potential overpressure, }\end{array}$ & A & $\mathrm{L}$ & $\mathrm{L}$ & $\mathrm{L}$ \\
\hline $1-37$ & \multicolumn{6}{|l|}{ Close V-109 and V-205 } \\
\hline $1-37.1$ & V-109 not closed & Hydrogen Deflagration: Sludge release to transfer box hydrogen buildup & $\bar{A}$ & $\mathrm{H}$ & $\mathrm{L}$ & $\mathrm{L}$ \\
\hline $1-37.2$ & V-109 not closed & Uncontrolled Release of Sludge: overpressure on transfer resulting in a spray or splash and splatter release of slurry: & A & $\mathrm{H}$ & $\mathrm{H}$ & $\mathrm{L}$ \\
\hline $1-50$ & \multicolumn{6}{|l|}{ Secure from 'test' mode (HS-701 to 'auto' position) } \\
\hline $1-50.1$ & $\begin{array}{l}\text { Not secured from 'test' mode (HS- } 701 \text { to 'auto' position) auto flush } \\
\text { from tank not actuated }\end{array}$ & $\begin{array}{l}\text { Uncontrolled Release of Sludge: potential to plug line in loss of power transfer shut down, overpressure on restart } \\
\text { resulting in a spray or splash and splatter release of slurry: }\end{array}$ & A & H & $\mathrm{H}$ & $\mathrm{L}$ \\
\hline $1-51$ & \multicolumn{6}{|c|}{ Close V-109 and AOV-104. Open V-101 and visually verify alignment of all manual valves in TLSB (V-101, V-102, V-103 open, all others closed) } \\
\hline $1-51.1$ & Valve mis-positioned Both Valves not closed & Hydrogen Deflagration: Sludge release to transfer box hydrogen buildup & A & $\mathrm{H}$ & $\mathrm{L}$ & $\mathrm{L}$ \\
\hline $1-51.2$ & Valve mis-positioned - Valve (V-101, or V-103) not opened & $\begin{array}{l}\text { Uncontrolled Release of Sludge: Deadhead transfer pump when started, potential overpressure, resulting in a spray or } \\
\text { splash and splatter release of slurry: }\end{array}$ & A & $\mathrm{H}$ & $\mathrm{H}$ & $\mathrm{L}$ \\
\hline $1-52$ & \multicolumn{6}{|c|}{ Close V-205. Open V-202, and visually verify alignment of all manual valves in decant box (V-201, V-202, V-204, V-210, V- 227 OPEN, all others closed) } \\
\hline $1-52.2$ & Valve not opened. & $\begin{array}{l}\text { Uncontrolled Release of Sludge: Deadhead transfer pump when started, potential overpressure, resulting in a spray or } \\
\text { splash and splatter release of slurry: }\end{array}$ & A & H & $\mathrm{H}$ & $\mathrm{L}$ \\
\hline \multicolumn{7}{|c|}{ Mode 8 DISCONNECT STSC } \\
\hline $8-29$ & \multicolumn{6}{|c|}{ Disconnect the Purge Outlet and install shipping Filter onto the 1 inch Quick Connect Nozzle F2 } \\
\hline $8-29.1$ & Fail to install filter & \multirow[b]{2}{*}{ Hydrogen Deflagration: Inerting degraded due to air exchange, hydrogen accumulation } & \multirow[b]{2}{*}{ A } & \multirow[b]{2}{*}{$\mathrm{H}$} & \multirow[b]{2}{*}{$\mathrm{L}$} & \multirow[b]{2}{*}{$\mathrm{L}$} \\
\hline $8-29.2$ & Take too long & & & & & \\
\hline $8-30$ & \multicolumn{6}{|c|}{ Disconnect the STSC Purge Gas inlet hose and install the shipping filter onto the 2 inch Quick Connect Nozzle S2 } \\
\hline
\end{tabular}


Table E-5 FREQUENCY AND CONSEQUENCE LEVEL RESULTS -GAP ANALYSIS SCENARIOS

\begin{tabular}{|c|c|c|c|c|c|c|}
\hline \multirow{2}{*}{ Identifier* } & \multirow{2}{*}{ What-If } & \multirow[b]{2}{*}{ Unmitigated Consequence } & \multirow{2}{*}{$\begin{array}{c}\text { Event } \\
\text { Freq }\end{array}$} & \multicolumn{3}{|c|}{ Consequence } \\
\hline & & & & FW & Onsite & Offsite \\
\hline $8-30.1$ & \begin{tabular}{|l|} 
Fail to install filter \\
\end{tabular} & \multirow[b]{2}{*}{ Hydrogen Deflagration: Inerting degraded due to air exchange, hydrogen accumulation } & \multirow[b]{2}{*}{ A } & \multirow[b]{2}{*}{$\mathrm{H}$} & \multirow[b]{2}{*}{$\mathrm{L}$} & \multirow[b]{2}{*}{$\mathrm{L}$} \\
\hline $8-30.2$ & Take too long & & & & & \\
\hline
\end{tabular}


PRC-STP-00467 REV 0

\section{APPENDIX F \\ HAZARDS ANALYSIS \\ PEER REVIEW CHECKLIST}

F-i 
PRC-STP-00467 REV 0

\section{CHPRC REVIEW CHECKLIST}

Document Reviewed:

PRC-STP-00473, Sludge Treatment Project Preliminary Design Hazard Analysis Supplement 1 reats Ramploz/1

\section{Scope of Review:}

The review was a peer review of the complete document.

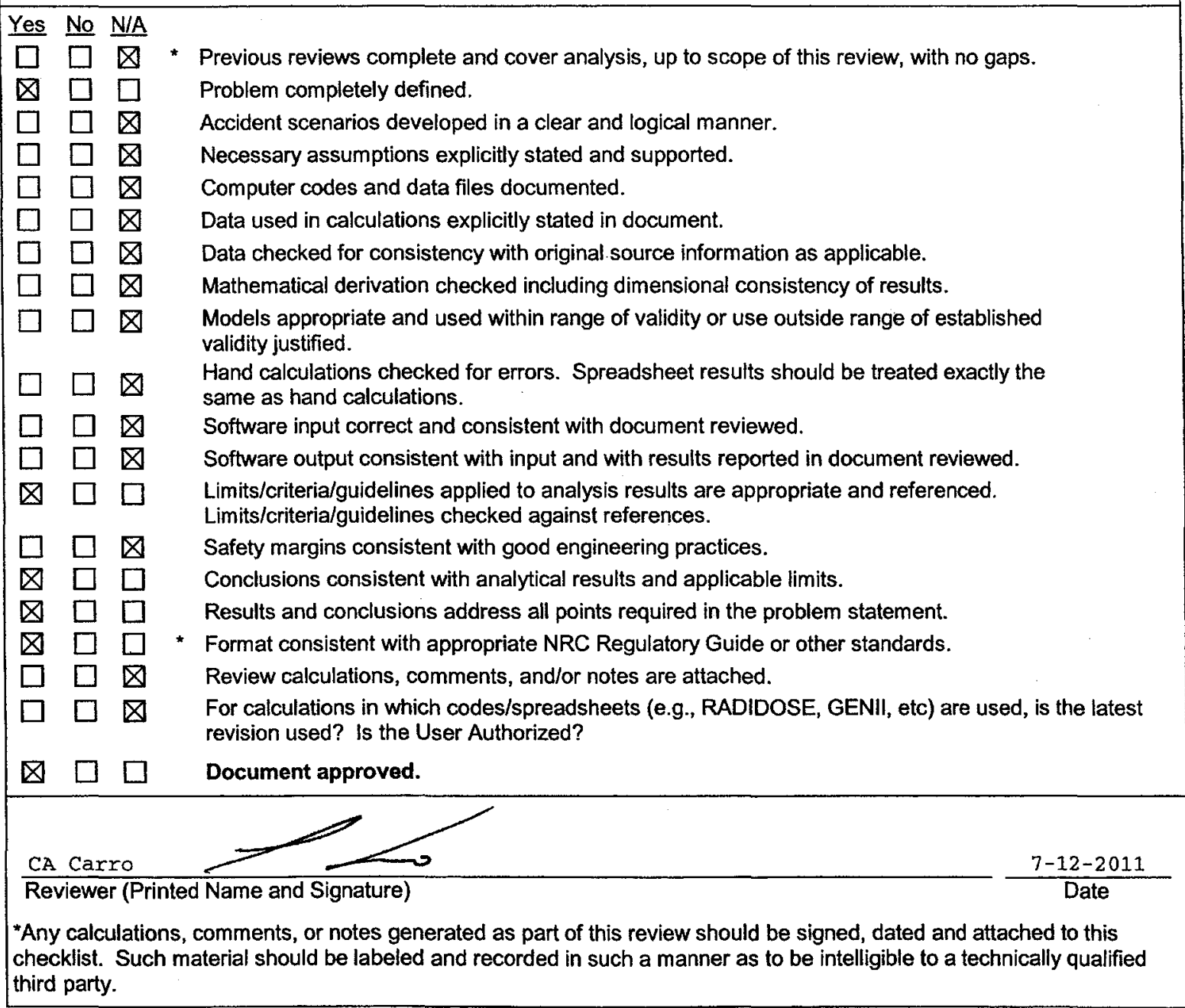

